\title{
Hidrodinámica Generalizada en Gases Densos de Enskog
}

\author{
Tesis que presenta \\ José Alejandro Rangel Huerta \\ para la obtención del grado de \\ Doctor en Ciencias \\ Física
}

Asesor:

Dra. Rosa Maria Velasco Belmont

Depto. de Física

CBI UAM-I

1996

Ciencias Básicas e Ingeniería

Universidad Autónoma Metropolitana-Iztapalapa 


\section{HIDRODINAMICA GENERALIZADA EN GASES \\ DENSOS DE ENSKOG}

Tesis que presenta

José Alejandro Rangel Huerta

para la obtención del grado de

Doctor en Ciencias

Física

Septiembre de 1996.

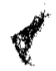


A:

Violeta y Maricruz

las mejores hijas 
Deseo expresar mi agradecimiento a la Dra. Rosa Ma. Velasco B., primero, por haberme dado la confianza de desarrollar este proyecto de investigación y luego por su paciencia, dedicación y apoyo siempre presentes a lo largo del desarrollo de esta tesis. Al Dr. Leopoldo García-Colín, Dr. Francisco Uribe, Dr. Luis F. del Castillo, Dr. Olegario Alarcón W., por la cuidadosa revisión y acertadas observaciones que han servido para precisar ideas y formular perspectivas de este trabajo. Al Departamento de Física de la División de C.B.I. por las facilidades que me brindó durante todo el periodo del Doctorado, a cuyos dedicados profesores les guardo una profunda admiración y respeto. Al CONACyT por el apoyo económico del cual disfruté, al menos parcialmente, durante mis estudios de posgrado. A mis compañeros del posgrado en Mecánica Estadística de la UAM-I, y del posgrado en el IFBUAP, de quienes siempre he recibido una colaboración valiosa y sincera amistad. A Beatriz Arce V. del Departamento de Matemáticas, por su gran ayuda y colaboración en la transcripción y presentación de este trabajo. 


\section{Indice}

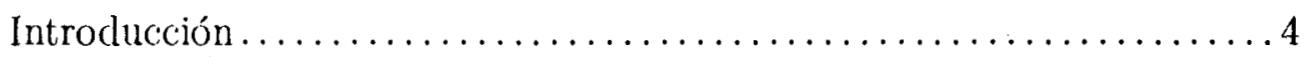

\section{CAPITULO 1}

Teoría Cinética de los Giases de Enskog. .....................9

1.1 La ecuación Cinética de Enskog..........................9

1.2 Transferencia Colisional ............................. 15

\section{CAPITULO 2}

Método de Momentos de Girad ...........................20

2.1 Variables relevantes................................... 21

2.2 La función de distribución (approximación a 13-momentos) ....... 24



2.4 Integrales constitutivas ............................. 30

2.5 Ecuaciones de movimiento linealizadlas .................... 33

CAPITULO 3

Coeficientes de Transporte generalizados

1er. Orden Flujos Colisionales ........................... 37

3.1 Ecuaciones de movimiento en el espacio- $(\vec{k}, \omega) \ldots \ldots \ldots \ldots \ldots \ldots 37$

3.2 Ecuaciones constitutivas generalizadas ................... 41

\section{CAPITULO 4}

Coeficientes de transporte generalizados

2do. Orden Flujos Colisionales ........................... 54 
4.1 Ecuaciones de movimiento en el espacio- $(\vec{k}, \omega) \ldots \ldots \ldots \ldots \ldots . \ldots 54$

4.2 Ecuaciones constitutivas generalizadas $\ldots \ldots \ldots \ldots \ldots \ldots \ldots \ldots$

4.3 Viscocidad volumétrica generalizada ..................6 65 CAPITULO 5

Hidrodinámica Generalizada ........................ 73

5.1 Ecuación de Langevin generalizada................... 73

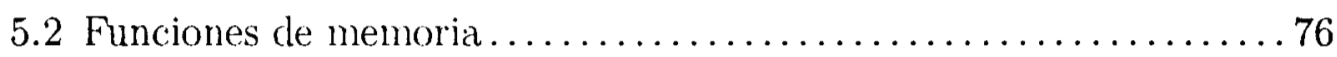

Concusiones y Perspectivas $\ldots \ldots \ldots \ldots \ldots \ldots \ldots \ldots \ldots \ldots \ldots \ldots$

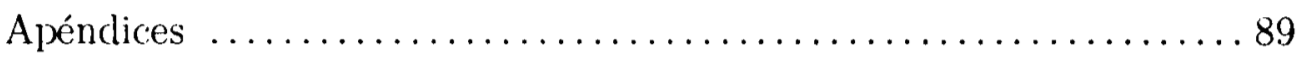

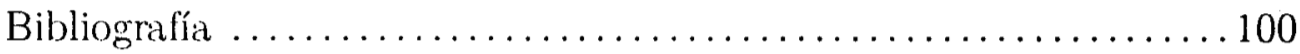




\section{Introducción}

El comportamiento hidrodinámico de un fluido simple presenta muchas facetas importantes que van desde la obtención del campo de velocidades bajo ciertas condiciones de frontera, hasta el estudio de las fluctuaciones espontáneas que ocurren en las variables que describen al sistema cuando éste se encuentra en un estado de equilibrio. En todos los casos son importantes las propiedades del fluido con el que se está trabajando y para una situación fuera del equilibrio termodinámico, los coeficientes de transporte son esenciales en la descripción.

Para la situación en que se estudian las fluctuaciones, dichos coeficientes están relacionados con funciones de correlación y son éstas las cantidades principales que se deben calcular a partir de la dinámica del problema.

La densidad espectral de las fluctuaciones en la densidad y algunas otras variables contienen toda la información relevante del sistema desde el punto de vista experimental. En general, estas funciones de correlación son difíciles de calcular directamente, sin embirgo, se pueden evaluar aproximadamente cerca del régimen hidrodinámico (bajas frecuencias y longitudes de onda larga), a partir de las ecuaciones hidrodinámicas de Navier-Stokes-Fourier, que describen la dinámica de un fluido viscoso. Los eigenvalores de la matriz hidrodinámica en el espacio Laplace-Fourier dan como resultado un espectro de dispersión con los primeros términos del desarrollo de frecuencias, a vector de onda fijo y pequeño, correspondiente a la suma de tres Lorentzianas. Esta representación gráfica se ajusta bastante bien al triplete de RayleighBrillouin que se obtiene en los experimentos de dispersión de ultrasonido y luz en fluidos monatómicos simples. En el caso de un tratamiento vía hidrodinámica clásica, los coeficientes de transporte son datos del problema ya sea que se obtengan mediante experimentos o bién que se tomen como resultado de algún modelo microscópico.

Existe una gran cantidad de tratamientos teóricos del tipo hidrodinámico [1-8], técnicas experimentales de absorción-dispersión [9-14] y simulaciones de dinámica molecular [15-20], enfocados a determinar el comportamiento dinámico de las fluctuaciones en regiones especificas de vectores de onda y frecuencias, así como intervalos característicos de densidad. Estos tratamientos en conjunto se denominan "hidrodinámica generalizada".

Un estudio detallado de las funciones de correlación temporal asociadas con las fluctuaciones de variables moleculares, nos proporciona mucha infor- 
mación sobre los detalles de los procesos dinámicos a nivel molecular en gases densos y líquidos. Las fluctuaciones tienen una distribución en longitudes de onda y frecuencias, así como una considerable dependencia con la densidad y temperatura del sistema.

El intervalo de frecuencias en los experimentos de dispersión de luz en sistemas densos, es mucho mayor al de dispersión de ultrasonido. Sin embargo, en frecuencias aún mayores correspondientes a los experimentos de dispersión de neutrones en líquidos o experimentos de dispersión de luz en gases, el análisis fundamentado sobre la hidrodinámica clásica usual ya no es válido, puesto que aparecen nuevas características del comportamiento del fluido en el régimen que llamaremos cinético y que corresponde al intervalo de estudio de la dinámica molecular microscópica.

Existen formalismos bien establecidos de la hidrodinámica generalizada, que van desde la ecuación de Langevin generalizada, teoría de la respuesta lineal, teoría de acoplamiento de modos, hasta las teorías cinéticas y técnicas de renormalización. Algunas de ellas son equivalentes, sus similitudes se pueden visualizar mejor en términos de la función de memoria, la cual tiene diferentes nombres en cada formalismo. La reducción del problema hidrodinámico general de las fluctuaciones, al de proponer un modelo fenomenológico sencillo para las funciones de memoria es muy importante al analizar la dinámica de fluidos.

Los formalismos generales de la hidrodinámica generalizada tienen como primera finalidad encontrar el origen microscópico de la dinámica molecular que es responsable del comportamiento hidrodinámico no-local y noinstantáneo de las fluctuaciones, mientras que un segundo objetivo pretende definir los procesos moleculares de relajación y transporte que gobiernan el comportamiento de las fluctuaciones en cleterminadas regiones del plano $(\vec{k}, \omega)$ para sistemas de densidad arbitraria [21-26].

Todo este estudio ha dado como resultado, principalmente, la comprobación teórica y experimental de la transición gradual y continua de la hidrodinámica descle el régimen hidrodinámico, de longitudes de onda larga y bajas frecuencias, hasta el régimen de dinámica molecular o régimen cinético [27-29]. La transición suave significa que podemos extender la hidrodinámica usual a la región finita, manteniendo la estructura de las ecuaciones linealizadas de Navier-Stokes pero tomando en cuenta las correlaciones microscópicas al incluir efectos no-locales y no-instantáneos en los coeficientes de transporte y parámetros termodinámicos, los cuales en general son funciones del vector 
de onda y la frecuencia.

Una de las conclusiones más importantes de la hidrodinámica generalizada indica que a nivel microscópico un fluido simple se comporta dinámicamente como si solamente tuviera algunos poco modos efectivos de relajación [20]. Los modos hidrodinámicos son los canales permitidos para los procesos de relajación en un fluido. En el régimen hidrodinámico las perturbaciones involucran un gran número de moléculas que realizan movimientos altamente correlacionados, denominados modos colectivos. Como ya hemos mencionado la existencia de modos colectivos de longitud de onda corta y alta frecuencia es una evidencia clara de la limitación de las ecuaciones de la hidrodinámica usual complementadas con coeficientes de transporte generalizados, funciones del vector de onda y la frecuencia [17-30-31].

La teoría cinética generalizarla de Enskog $[17,30-31]$, ha permiticlo fundamentar la hidrodinámica generalizada y estudiar la transición del régimen hidrodinámico hacia la región $(\vec{k}, \omega)$-finito de manera unificada. El resultado básico de la teoría es que el fluido se comporta dinámicamente como si solamente tuviese algunos eigenmodos efectivos, aún en el régimen de dinámica molecular. Esto puede considerarse como una extensión de los modelos usuales de la hidrodinámica para construir una teoría generalizadla. Los resultados demuestran que para fluidos densos las ecuaciones de Navier-Stokes son válidas para vectores de onda tales que $k a \lesssim 1$, y que para $1 \leqslant k a \lesssim 30$ las continuaciones analíticas de los modos hidrodinámicos siguen describiendo los rasgos principales del factor de estructura dinámico. Otra conclusión importante es que se pueden establecer tres regiones de densidad, caracterizados por la densidad reducidla $\delta=n a^{3}$, donde $n$ es la densidad de número y a corresponde al diámetro de la esfera dura. El gas diluido $(\delta<0.1)$, gas moderadamente denso $(0.1<\delta<0.35)$ y el régimen del gas denso $(0.35<\delta<0.70)[25,27,30,37]$.

En la práctica los coeficientes de transporte generalizados (CTG) se proponen en forma empírica mediante modelos razonables con parámetros libres que se ajustan exigiendo, como restricción, que se cumplan las reglas de suma y que en el régimen hidrodinámico se recuperen los coeficientes usuales. Los parámetros libres se identifican con algunos tiempos de relajación, y aún cuando podemos describir adecuadamente él decaimiento de todas las funciones de memoria relevantes, permanece el carácter fenomenológico de los modelos utilizados. 
Introduciendo una escala espacio-temporal en la hidrodinámica usual, podemos obtener una descripción hidrodinámica generalizada que permita considerar de manera unificada las mediciones de dispersión inelástica de luz y neutrones, es decir, considerar una región finita de vectores de onda y frecuencias a partir del régimen hidrodinámico. Este procedimiento alternativo, también de carácter fenomenológico, consiste en generalizar las relaciones constitutivas de tal forma que la respuesta de las fuerzas termodinámicas, vistas como flujos disipativos del fluido, es no-local y no-instantánea. Muchos esfuerzos se han hecho para demostrar que la forma de las ecuaciones de la hidrodinámica usual se pueden mantener en la región finita de vectores de onda y frecuencias, mediante la introducción de coeficientes de transporte generalizados, esto es un reflejo de las correlaciones espacio-temporales involucradas en la disipación de ímpetu y energía a nivel molecular y que estos procesos evolucionan más rápido que los tiempos asociaclos con el decaimiento de variables conservadas. Excepto por los resultados de simulación de dinámica molecular, no existe una deducción microscópica de los coeficientes de transporte generalizado a densidades elevadas [32-36]. La evaluación teórica presenta un problema muy complejo, podemos hacer un análisis de fluctuación disipación lineal para formular expresiones generales de las funciones de correlación válidas para coeficientes generalizados, a cualquier densidad, temperatura y potencial molecular, sin embargo, su evaluación requiere necesariamente de la solución del problema de muchos cuerpos.

Ahora bien, nos preguntamos ¿,Cómo podemos calcular, a partir de los procesos dinámicos a nivel molecular, los coeficientes de transporte generalizados en función del vector de onda y la frecuencia para un fluido simple?

La respuesta a esta pregunta se ha dado principalmente a través de la teoría cinética de gases $[38,39]$, en la cual la interacción entre partículas a nivel molecular adquiere relevancia. Para gases diluidos formados por átomos, la ecuación de Boltzmann proporciona respuestas adecuadas y en la literatura se han calculado los coeficientes de viscosidad cortante generalizada, conductividad térmica generalizada, coeficientes de termostricción, etc. Sin embargo, esto sólo es válido en un régimen de densidad pequeña, de manera que una preocupación existente es la generalización de estos métodos al régimen del gas denso. En este camino se han encontrado numerosas dificultades, que de hecho no están resueltas de manera satisfactoria.

En este trabajo estamos interesados en desarrollar un modelo cinético que permita obtener coeficientes de transporte generalizados para un gas simple 
moderadamente denso, y algunas de sus consecuencias más immediatas. Una vez que contamos con tales coeficientes podemos regresar a las aplicaciones, donde pueden utilizarse en problemas hidrodinámicos, desde el régimen cinético hasta el hichrodinámico.

La teoría cinética estándar de Enskog (SET), complementada con el método de momentos de Grad nos permite estudiar el comportamiento de un gas denso de esferas duras, en el régimen de longitudes de onda corta y altas frecuencias. El cálculo se desarrolla con la aplicación del método de solución de Grad a la ecuación cinética, cuya reducción al espacio de variables conservadas nos permite establecer relaciones constitutivas generalizadas, que definen a los coeficientes de transporte en función del vector de onda y la frecuencia. Estos últimos por cierto, serán los elementos de la matriz de memoria en el esquema de la ecuación de Langevin generalizada. En todo el desarrollo estaremos considerando los resultados correspondientes a gases diluidos, los cuales suponemos conocidos, constituyendo el marco de referencia de las discusiones posteriores. 


\section{Capítulo 1}

\section{Teoría Cinética de los Gases de Enskog}

La problemática involucradia al tratar de obtener una ecuación cinética que describa el comportamiento de gases densos, ha resultado ser una tarea muy compleja, que de hecho no ha sido acabada [40-43]. Entre las ecuaciones cinéticas existentes, se tiene la ecuación de Enskog [44-47] que es válida para gases moderadamente densos cuyas moléculas interactúan a través de un potencial de esfera dura. Esta ecuación a pesar de que no está bien fundamentada proporciona una guía para el estudio del comportamiento cinético de los gases densos [57-58]. Más aún, jor razones a veces no muy bien entendidas esta ecuación proporciona resultados buenos, para los coeficientes de transporte en el régimen hidrodinámico y en intervalos de densidad cercanos a los del líquido [50-51]. En esta sección presentamos los rasgos principales de la ecuación de Enskog, y la comparamos con otras ecuaciones cinéticas para gases densos [48-49]. También obtenemos una ecuación cle balance general, principalmente porque éste es el origen formal de los flujos colisionales y porque esta ecuación de balance será fundamental para la descripción hidrodinámica del gas.

\subsection{La Ecuación Cinética de Enskog}

En el tratamiento subsecuente vamos a utilizar la ecuación cinética de Enskog (EE) $[44,47]$, para describir el comportamiento temporal de gases monoatómicos de esferas duras, que denominaremos "Gases de Enskog". El estado cinético del gas está caracterizado por una función de distribución de una partícula $f(\vec{x}, \vec{c}, t)$, cle manera que $f(\vec{x}, \vec{c}, t) d \vec{x} d \vec{c}$ es el número de moléculas en el elemento de volumen entre $\vec{x}$ y $\vec{x}+d \vec{x}$, y con velocidades entre $\vec{c}$ y $\vec{c}+d \vec{c}$ al tiempo t. Esta función representa la densidad numérica para los puntos en el espacio- $(\vec{x}, \vec{c})$, y evoluciona en el tiempo de acuerdo con una ecuación de movimiento, la cual en ausencia de fuerzas externas, puede representarse en la forma siguiente:

$$
\frac{\partial f}{\partial t}+c_{i} \frac{\partial f}{\partial x_{i}}=J_{E}(f, f)
$$


La ec.(1.1) nos dice como (ambia $f$ en el tiempo, debido al movimiento libre de las moléculas y a las interacciones entre las mismas. El término que mide las interacciones está representado por $J_{E}(f, f)$ y su expresión depende fuertemente del modelo molecular. Para la ecuación de Enskog suponemos que las moléculas son esferas perfectamente elásticas, rígiclas y lisas. En este caso, las colisiones moleculares son instantáneas y podemos despreciar las colisiones moleculares múltiples, considerando que la dinámica del sistema queda completamente determinada por colisiones binarias.

La ecuación cinética que resulta es muy semejante a la ecuación de Boltzmann, sin embargo, se deben de considerar dos modificaciones may importantes:

i) Aún cuando no se consideran correlaciones entre las velocidades moleculares se toman en cuenta de manera aproximada, las correlaciones espaciales. La manera de introducir estas correlaciones es cualitativa y se basa en la observación de que en un gas denso, la probabilidad de colisión entre partículas aumenta. Así, al escribir el término de colisión de la misma forma que en la ecuación de Boltzmann, el producto de las dos funciones de distribución está multiplicado por un factor $\chi$, que es función de la densidad local y por razones de simetría debe estar evaluado en el punto de contacto de las dos esferas en colisión.

El aumento en la frecuencia de colisiónes se debe a la reducción del volumen accesible a cualquier molécula por la presencia de las otras y corresponde a lo que en estados de equilibrio se conoce como "volumen excluido".

ii) Por otra parte, en un gas denso debemos tomar en cuenta la variación de la función de distribución en distancias del orden de un diámetro molecular, ésto conduce a colisiones moleculares no-localizadas. Es decir, las dos funciones de clistribución del término colisional están evaluadas en puntos diferentes, donde se encuentran los centros moleculares.

Mediante un procedimiento muy parecido a la deducción de la ecuación de 
Boltzmann, y con ayuda de las hipótesis anteriores obtenemos:

$$
\begin{aligned}
\frac{\partial f}{\partial t}+c_{i} \frac{\partial f}{\partial x_{i}}= & \int\left[\chi\left(\vec{x}+\frac{a}{2} \hat{k}, t\right) f^{\prime}(x) f_{1}^{\prime}(\vec{x}+a \hat{k})-\right. \\
& \left.-\chi\left(\vec{x}-\frac{a}{2} \hat{k}, t\right) f(\vec{x})-f_{1}(\vec{x}-a \hat{k})\right] a^{2}(\vec{g} \cdot \hat{k}) d \hat{k} \vec{c}_{1},
\end{aligned}
$$

en esta ecuación hemos introducido las abreviaciones siguientes:

$$
\begin{aligned}
f^{\prime}(\vec{x}) \equiv f(\vec{x}, \vec{c}, t) & f_{1}^{\prime}(\vec{x}+a \hat{k}) \equiv f\left(\vec{x}+a \hat{k}, \vec{c}_{1}, t\right) \\
f(\vec{x}) \equiv f(\vec{x}, \vec{c}, t) & f_{1}(\vec{x}-a \hat{k}) \equiv f\left(\vec{x}-a \hat{k}, \vec{c}_{1}, t\right) .
\end{aligned}
$$

Hemos denotado por $\left(\vec{c}, \vec{c}_{1}\right)$ y $\left(\vec{c}, \vec{c}_{1}\right)$ a las velocidades moleculares, antes y después de la colisión respectivamente. Estas no son inclependientes, se encuentran relacionadas por la conservación del ímpetu

$$
\vec{c}^{\prime}=\vec{c}+(\vec{g} \cdot \hat{k}) \hat{k}, \quad \vec{c}_{1}^{\prime}=\vec{c}_{1}-(\vec{g} \cdot \hat{k}) \hat{k}
$$

donde $\vec{g}=\vec{c}_{1}-\vec{c}$ es la velocidlad relativa y $\hat{k}$ es un vector unitario en la dirección de la línea que une los dos centros de las moléculas que chocan. Este vector se escoge de manera que apunte desde la molécula etiquetada con 1 hacia la otra, es decir, hacia la molécula de referencia. Más aún, $a$ es el diámetro molecular y $d \hat{k} \equiv \operatorname{sen} \theta d \theta d c ; 0 \leq \theta \leq \pi / 2$ es el ángulo entre $\vec{g} \mathrm{y}$ $\hat{k}$; mientras que $0 \leq \epsilon \leq 2 \pi$ es el ángulo entre el plano que contiene a $\vec{g}$ y $\hat{k}$ con un plano de referencia que contiene a $\vec{g}$.

Para representar la frecuencia de las colisiones en la teoría de Enskog, se propone que el factor $\chi \equiv \chi(\vec{x}, \vec{x}+a \hat{k})$ sea la función de correlación por pares fuera de equilibrio para dos partículas en contacto. Como la forma de esta función no es conocida, se supone que la dependencia de $\chi$ con la densidad es como la de $[48,49] g(a)$ la función de correlación por pares en equilibrio, sin embargo, la densidad usada para $\chi$ es la densidad local en un punto medio entre las dos esferas: $n\left(\vec{x}+\frac{1}{2} a \hat{k}\right)$.

Debido a que $g(a)$ es la función cle correlación por pares en equilibrio, tiene un desarrollo en la densidad de la forma [48]:

$$
\begin{aligned}
g(a)= & 1+\frac{5}{8} b n_{0}+0.28695\left(b n_{0}\right)^{2}+0.1103\left(b n_{0}\right)^{3} \\
& +0.0386\left(b n_{0}\right)^{4}+0.0138\left(b n_{0}\right)^{5}+\cdots
\end{aligned}
$$


donde $n_{0}$ es la densidad numérica en equilibrio y $b=\frac{2}{3} \pi a^{3}$ el covolumen.

La suposición que se hace en la teoría de Enskog, es que $\chi(\vec{x}, \vec{x}+a \hat{k})$ esta dada por:

$$
\begin{aligned}
\chi(\vec{x}, \vec{x}+a \hat{k})= & 1+\frac{5}{8} b n\left(\vec{x}+\frac{1}{2} a \hat{k}\right)+0.28695 \times \\
& \times\left[b n\left(\vec{x}+\frac{1}{2} a \hat{k}\right)\right]^{2}+0.1103\left[b n\left(\vec{x}+\frac{1}{2} a \hat{k}\right)\right]^{3}+ \\
& +0.0386\left[b n\left(\vec{x}+\frac{1}{2} a \hat{k}\right)\right]+0.0138\left[b n\left(\vec{x}+\frac{1}{2} a \hat{k}\right)\right]^{5}+\cdots
\end{aligned}
$$

Esta expresión para la función de correlación por pares fuera de equilibrio se sustenta en la suposición de que aún en un estado fuera de equilibrio la función de correlación debe estar determinada únicamente por el requisito físico de que las moléculas esféricas no se traslapan. Así, $\chi(\vec{x}, \vec{x}+a \hat{k})$ debe tomar en cuenta la misma clase de efectos de volumen excluido como los que se incorporan en equilibrio a través de $g(a)[52,53\}$.

En resumen, la función $\chi$ se evalía usando $g(a)$, es independiente de la temperatura y solamente depende de la densidad local evaluada en el punto contacto; es por ello que admite un desarrollo virial en equilibrio local. Esta función representa una correlación espacial molecular de corto alcance, puesto que la mayor influencia corresponde a los vecinos más cercanos a la molécula de referencia.

La exposición anterior corresponde a lo que se conoce como la "teoría estandard de Enskog" (SET). Este tratamiento solamente es la primera aproximación a una ecuación cinética para gases densos.

En la "Teoría modificada de Enskog" (MET) [48-49] se considera que el factor $\chi$ es estrictamente igual a la función de distribución en equilibriolocal, la cual contiene todas las inhomogeneidades espaciales del sistema. El desarrollo modificado de $\chi_{M}$ debe ser

$$
\begin{aligned}
\chi_{M}\left(\vec{x}_{1}, \vec{x}_{2}\right)= & 1+\int d \vec{x}_{3} n\left(\vec{x}_{3}, t\right) V\left(\vec{x}_{1}, \vec{x}_{2} \mid \vec{x}_{3}\right)+ \\
& +\frac{1}{2} \int d \vec{x}_{3} \int d \vec{x}_{4} n\left(\vec{x}_{3}, t\right) n\left(\vec{x}_{4}, t\right) V\left(\vec{x}_{1}, \vec{x}_{2} \mid \vec{x}_{3}, \vec{x}_{4}\right)+\cdots
\end{aligned}
$$


donde

$$
V\left(\vec{x}_{1}, \vec{x}_{2} \mid \vec{x}_{3}\right)=F\left(\vec{x}_{1}, \vec{x}_{3}\right) F\left(\vec{x}_{2}, \vec{x}_{3}\right)
$$

y la $F$ es la función de Mayer $F\left(\vec{x}_{i}, \vec{x}_{j}\right) ; i, j=1,2,3$ que está dada por

$$
F\left(\vec{x}_{i}, \vec{x}_{j}\right)=e^{-\beta \phi\left(\left|\vec{x}_{i}-\vec{x}_{j}\right|\right)}-1,
$$

etc. y $\phi$ es el potencial de interacción entre partículas, de tal forma que $\chi_{M}(\vec{x}, \vec{x} \pm a \hat{k})$ se obtiene al hacer $\vec{x}_{1}=\vec{x} ; \vec{x}_{2}=\vec{x} \pm a \hat{k}$.

La ecuación cinética que proporciona la MET, se puede obtener a partir de hipótesis razonables sobre la jerarquía BBGKY. Formalmente, la ecuación cinética de MET, coincide con la EE de la ecuación (1.2), excepto que $\chi$ es reemplazadla por $\chi_{M}$.

La teoría generalizada de Enskog incorpora a la ecuación cinética una sucesión de colisiones binarias relevantes, que son sugeridas por los cálculos de dinámica molecular (DM). La correlación correspondiente se extiende a distancias mucho mayores que el-diámetro molecular, de tal forma que dominan el comportamiento colectivo del fluido. Esta teoría cinética híbrida con D.M. ha permitido la unificación del comportamiento cinético e hidrodinámico generalizado en términos de los modos hidrodinámicos extendidos.

Con el desarrollo de las técnicas computacionales se ha optado por combinar los cálculos de Dinámica Molecular con un tratamiento no-sistemático de la teoría cinética. Este método híbrido permite incorporar solamente las características relevantes de la sucesión de colisiones binarias necesarias para entender el comportamiento colectivo del fluido y reproducir los resultados experimentales.

Recientemente se ha elaborado una generalización de la SET para gases densos con interacción de Lennard-Jones [42-43]. Para este sistema parece imposible identificar colisiones, sin embargo, haciendo la hipótesis de interacción por pares se encuentra una generalización de la SET que tiene una ecuación cinética fundamental;

$$
\frac{\partial f}{\partial t}+c_{i} \frac{\partial}{\partial x_{i}} f=\int_{0}^{t} d t^{\prime} \int d \vec{x}^{\prime} \int d \vec{c} T\left(\vec{x}-\vec{x}^{\prime}, \vec{c}, \vec{c}^{\prime}, t-t^{\prime}\right) f^{\prime},
$$

donde:

$$
f \equiv f(\vec{x}, \vec{c}, t), \quad f^{\prime}\left(\vec{x}^{\prime}, \vec{c}^{\prime}, t^{\prime}\right) .
$$

El problema es hallar una buena aproximación para el operador $T$, sugerida por Dinámica Molecular y que contenga las correlaciones espaciotemporales. 
Esta ecuación cinética describe a las partículas de referencia, interactuando mediante un potencial de L.J. y puede ser utilizada para calcular la función de autocorrelación de las velocidlades. Los resultados obtenidos con éste método nos permiten estimar el valor de la densidad para que el modelo de colisiones binarias sea válido y nos pueden decir cuales son los intervalos de densidad en los que es aplicable el modelo de colisiones binarias.

Cuando suponemos que la hipótesis de colisión binaria es adecuada para describir la evolución temporal de un fluido L.J., debemos considerar que las colisiones ocurren en un tiempo finito. El intercambio de energía cinética y potencial en clichas interacciones moleculares es un proceso muy complejo. De manera cualitativa los resultados parecen indicar que el mecanismo dominante en el intercambio de energía son las colisiones binarias entre la parte repulsiva del potencial, perturbadas por la presencia de terceras partículas. En altas densidades las colisiones repeticlas, se pueden confundir con las colisiones perturbadas en la parte dura del potencial, y por lo tanto la definición de colisión molecular pierde senticlo.

Por otra parte, este tratamiento ha demostrado que satisface ecuaciones macroscópicas de conservación, aunque no se ha utilizado para hacer estudios hidrodinámicos y sólo parcialmente se ha incluido a los coeficientes de transporte en su descripción.

Estas son sólo algunas de las generalizaciones que se obtienen directamente de la SET, y que hacen posible una descripción satisfactoria de gases densos simples. Existen otros tratamientos más generales en su planteamiento, que incluyen modelos moleculares más elaborados y que por lo tanto pueden describir otras características relevantes de los gases clensos; pero en todas ellas se trata de no perder las simplificaciones y beneficios que se tienen con los modelos tipo Enskog [54-56].

En este trabajo estamos interesados principalmente en obtener coeficientes de transporte generalizados para gases de Enskog, en una región espaciotemporal donde es válida la Hidrodinámica Generalizada basándonos en los resultados obtenidos en el régimen cliluido, pensamos que la "Teoría estandard de Enskog" puede servir para este fin en base a los resultados que se han obtenido en el régimen hidrodinámico. Pensamos que en términos generales el interés principal al tratar con gases de Enskog, radica en el hecho de que las propiedarles de transporte dependen esencialmente de la existencia de las colisiones moleculares más que de su naturaleza. 


\subsection{Transferencia Colisional}

En gases densos y líquidos el mecanismo de transporte denominarlo "Transferencia Colisional", juega un papel muy importante y de hecho es el mecanismo dominante para los procesos de transporte de masa, momento y energía.

Para entender con claridad este concepto, primero nos referimos al modelo molecular de esfera dura. Cada una de las moléculas en el sistema transporta masa, momento y energía por el sólo hecho de moverse, y si fijamos nuestra atención a un plano particular del sistema podremos contar la cantidad de estas propiedades físicas que han sido transferidas de un lado del plano al otro. A este tipo de transferencia le llamaremos transporte convectivo y siempre está presente independientemente de la densidad.

Cuando hay transferencia de estas propiedades a través del plano de referencia sin que las partículas pasen de un lado a otro del plano se tiene la transferencia colisional. Es claro que si la clensidad del sistema es considerable, dicha transferencia juega un papel muy importante. Esto es debido a que las partículas tienen poco espacio para moverse libremente y entonces el transporte convectivo se ve reducido. No sucede así en la transferencia colisional ya que las colisiones son muy frecuentes y habría contribuciones a la transferencia sin un recorrido apreciable de las partículas. Debe quedar claro que ambos mecanismos de transporte siempre están presentes, sólo que en un gas diluido es más importante el transporte convectivo y en un gas denso lo es el transporte colisional.

Cuando las partículas interactúan a través de un potencial central, podemos visualizar estos mecanismos de una manera un poco más general.

Considerando que las moléculas son centros de fuerzas, caracterizados por un potencial radial de corto alcance, la colisión ahora puede verse como una dispersión de partículas en el esquema de fuerzas centrales.

En esta dispersión se realiza un "intercambio" molecular de ímpetu y energía a "distancia", ello en una región considerable de la trayectoria. La dispersión se realiza en un tiempo finito, durante el cual podemos considerar que hay una transferencia de energías cinética y potencial. El flujo que se genera de esta manera, de hecho ocurre a través del potencial, de ahí que a la transferencia colisional se le identifique con un flujo potencial de las cantidades correspondientes.

Este mecanismo es dominante en gases densos y es más eficiente que el 
transporte por movimiento molecular. Es muy importante distinguir entre el mecanismo de transporte y las propiedades mismas que se intercambian en las colisiones moleculares por medio de flujos a través del espacio [44-47].

En gases de Enskog, las colisiones de esfera clura son elásticas y no localizadas. Esto genera un flujo instantáneo de ímpetu y energía cinética a través del espacio entre los dos centros de las moléculas en colisión. El potencial molecular de esfera dura (geometría esférica) permite que la colisión tenga lugar a "distancia" y el carácter elástico de la misma hace posible un intercambio de propiedades moleculares traslacionales (funciones de la velocidad) a través del espacio.

Descle un punto de vista formal, la transferencia vía colisión se obtiene a partir de la ecuación de Enskog al desarrollar en serie de Taylor las funciones de distribución que intervienen en el término de colisión. De hecho el segundo miembro de la ecuación (1.2) se escribe generalmente como la suma de tres contribuciones, de donde la EE se-puede expresar como,

$$
\frac{\partial f}{\partial t}+c_{i} \frac{\partial f}{\partial x_{i}}=J^{0}(f f)+J^{I}(f f)+J^{I I}(f f),
$$

donde,

$$
\begin{aligned}
J^{0}(f f)= & \int \chi\left(f_{1}^{\prime} f^{\prime}-f_{1} f\right) a^{2}(\vec{g} \cdot \hat{k}) d \hat{k} d \vec{c}_{1}, \\
J^{I}(f f)= & a \int\left\{\chi\left[f^{\prime} \frac{\partial f_{1}^{\prime}}{\partial x_{i}}+f \frac{\partial f_{1}}{\partial x_{i}}\right]+\right. \\
& \left.+\frac{1}{2} \frac{\partial \chi}{\partial x_{i}}\left(f_{1}^{\prime} f^{\prime}+f_{1} f\right)\right\} k_{i} a^{2}(\vec{g} \cdot \hat{k}) d \hat{k} d \vec{c}_{1} \\
J^{I I}(f f)= & \frac{a^{2}}{2} \int\left\{\chi\left[f^{\prime} \frac{\partial^{2} f_{1}^{\prime}}{\partial x_{i} \partial x_{j}}-f \frac{\partial^{2} f_{1}}{\partial x_{i} \partial x_{j}}\right]+\right. \\
& +\frac{\partial x}{\partial x_{i}}\left[f^{\prime} \frac{\partial f_{1}^{\prime}}{\partial x_{j}}-f \frac{\partial f_{1}}{\partial x_{j}}\right] \\
& \left.+\frac{1}{4} \frac{\partial^{2} \chi}{\partial x_{i} \partial x_{j}}\left(f_{1}^{\prime} f^{\prime}-f_{1} f\right)\right\} \cdot k_{i} k_{j} a^{2}(\vec{g} \cdot \hat{k}) d \hat{k} d \vec{c}_{1} .
\end{aligned}
$$


En las ecuaciones (1.12) todas las funciones que aparecen: $f, f_{1}, f^{\prime}, f_{1}^{\prime}$ y $\chi$, están evaluadas en $\vec{x}$. Notemos que $J^{0}(f f)$ con $\chi=1$ es el término de colisión usual de la EB para gases diluidos. $J^{I}(f f)$ incluye sólo gradientes de primer orden, mientras que $J^{I I}(f f)$ contiene gradientes de segundo orden, así como productos de gradientes.

La ecuación de transferencia que contiene flujos por "transferencia colisional" se obtiene multiplicando la ecuación (1.12) por una función arbitraria $\psi(\vec{x}, \vec{c}, t)$, no necesariamente un invariante colisional, e integrando sobre todos los valores de $\vec{c}$. Esta ecuación puede ser escrita en forma simplificada [54-56]:

$$
\frac{\partial \Psi}{\partial t}+\frac{\partial}{\partial x_{i}}\left(\phi_{i}^{0}+\phi_{i}^{I}+\phi_{i}^{I I}\right)=P^{0}+P^{I}+P^{I I},
$$

- $\Psi$ es la clensidad local macroscópica de $\psi$.

- $\phi_{i}^{0}$ clensidlad de flujo por traslación molecular (Parte Cinética del flujo).

- $\phi_{i}^{I}, \phi_{i}^{I I}$ Densidad de flujo por colisiones moleculares. Transferencia colisional (Parte potencial del flujo).

- $P^{0}, P^{I}, P^{I I}$ Términos de producción.

Estas cantidades están definidas por:

$$
\psi=\int \psi f d \vec{c}, \quad \phi_{i}^{0}=\int \psi c_{i} f d \vec{c}
$$




$$
\begin{aligned}
\phi_{i}^{I}= & \frac{a}{2} \int \chi\left(\psi^{\prime}-\psi\right) k_{i} f f_{1} d \Gamma \\
\phi_{i}^{I I}= & \frac{a^{2}}{4}\left\{\int \chi\left[\left(\psi^{\prime}-\psi\right) \frac{\partial}{\partial x_{j}}\left(\ln \frac{f}{f_{1}}\right)+\frac{\left(\partial \psi^{\prime}-\psi\right)}{\partial x_{j}}\right] k_{i} k_{j} f f_{1} d \Gamma-\right. \\
& \left.-\frac{1}{2} \frac{\partial}{\partial x_{j}} \int \chi\left(\psi^{\prime}-\psi\right) f f_{1} k_{i} k_{j} d \Gamma\right\} \\
P^{0}= & \int\left[\frac{\partial \psi}{\partial t}+c_{i} \frac{\partial \psi}{\partial x_{i}}\right] f d \vec{c}+\int \chi\left(\psi^{\prime}-\psi\right) f f_{1} d \Gamma \\
P^{I}= & \frac{a}{2} \int \chi\left[\left(\psi^{\prime}-\psi\right) \frac{\partial}{\partial x_{i}}\left(\ln \frac{f}{f_{1}}\right)+\frac{\partial\left(\psi^{\prime}-\psi\right)}{\partial x_{i}}\right] k_{i} f f_{1} d \Gamma, \\
P^{I I}= & \frac{a^{2}}{4} \int \chi\left\{\frac{\partial\left(\psi^{\prime}-\psi\right)}{\partial x_{i}} \frac{\partial}{\partial x_{j}}\left(\ln \frac{f}{f_{1}}\right)+\frac{1}{2} \frac{\partial^{2}\left(\psi^{\prime}-\psi\right)}{\partial x_{i} \partial x_{j}}-\right. \\
& -\left(\psi^{\prime}-\psi\left(\psi^{\prime}\right) \frac{\partial \ln f}{\partial x_{i}} \frac{\partial \ln f_{1}}{\partial x_{j}}+\frac{1}{2}\left(\psi^{\prime}-\psi\right)\left[\frac{1}{f} \frac{\partial^{2} f}{\partial x_{i} \partial x_{j}}+\right.\right. \\
& +\frac{1}{f_{1}} \frac{\partial^{2} f_{1}}{\left.\left.\partial x_{i} \partial x_{j}\right]\right\} k_{i} k_{j} f f_{1} d \Gamma .}
\end{aligned}
$$

donde, $d \Gamma=a^{2}(\vec{g} \cdot \hat{k}) d \hat{k} d \vec{c} d \vec{c}_{1}$. La expresión $\left(\psi^{\prime}-\psi\right)$ en los últimos términos se puede reemplazar por

$$
\frac{1}{2}\left(\psi_{1}^{\prime}-\psi_{1}+\psi^{\prime}-\psi\right)
$$

que permite ver con claridad que si la función $\psi$ corresponde a una cantidad invariante en la colisión, la contribución a estas integrales se anula. A su vez esta sustitución permite en algunos casos efectuar las integraciones de manera más conveniente.

La ecuación en (1.13) nos describe como es la evolución temporal de la densidad macroscópica $\Psi$. Toma en cuenta tanto la transferencia convectiva como la colisional y tiene la forma general de una ecuación de balance, donde aparecen los flujos y las fuentes correspondientes. Por el momento esta ecuación de transporte general tiene las limitaciones propias de la ecuación 
de Enskog y de los desarrollos en serie de Taylor, mismos que son equivalentes a los desarrollos en la densidad, como veremos posteriormente de manera más explícita. 


\section{Capítulo 2}

\section{Método de momentos de Grad}

Para resolver la ecuación de Enskog, podemos utilizar los métodos de solución que se aplican a la ecuación de Boltzmann para gases diluidos. Las soluciones normales que se obtienen con el método de Chapman-Enskog [4446], solamente se aplican cerca del régimen hidrodinámico, de longitudes de onda larga y baja frecuencia. Por eso no se pueden aplicar completamente a fenómenos tan importantes como, por ejemplo, ondas de choque robustas, condiciones de frontera en flujos de capa límite e hidrodinámica generalizada.

En cambio, el método de momentos de Grad [59-60], constituye un método general para obtener las soluciones no-normales de la ecuación de Boltzmann, es relativamente simple en su concepción, aunque muy complicado cuando se consideran los detalles de su aplicación. Este método se sustenta en el hecho de que los polinomios multidimensionales de Hermite forman una base completa y entonces, la función de distribución puede escribirse como una combinación de ellos y coeficientes que quedan por determinar.

Cuando estas ideas se aplican a la función de distribución, solución de la EE, se trabaja en el espacio de las velocidades de manera que la dependencia de la función de distribución con la velocidad queda determinada por el conjunto de polinomios.

Los coeficientes del desarrollo son los momentos de la función de distribución que dependen solamente de las coordenadas espaciales y del tiempo. Los polinomios multidimensionales de Hermite representan los polinomios equivalentes cartesianos de Somnine, son ortonormales y la función de peso a partir de la cual se pueden generar corresponde a una función gaussiana.

Esto nos permite incorporar a los flujos como variables relevantes en la descripción del sistema, mediante un conjunto de ecuaciones de evolución que provienen directamente de la ecuación cinética. En lo que sigue vamos a obtener la aproximación a 13-momentos de la EE considerando flujos colisionales de segundo orden en las ecuaciones de movimiento de las variables relevantes del sistema. 


\subsection{Variables relevantes}

El estado microscópico de un gas de Enskog está caracterizado por una función de clistribución de partícula simple $f(\vec{x}, \vec{c}, t)$, la cual evoluciona en el tiempo de acuerdo con la ecuación cinética de Enskog. Esta función de distribución queda completamente determinada por todos sus momentos con respecto a la velocidad molecular. Algunos de ellos son:

$$
n(\vec{x}, t)=\int f(\vec{x}, \vec{c}, t) d \vec{c},
$$

la densidad numérica corresponde a un momento de orden cero.

$$
u_{i}(\vec{x}, t)=\frac{1}{n(\vec{x}, t)} \int c_{i} f(\vec{x}, \vec{c}, t) d \vec{c},
$$

la velocidad hidrodinámica, momento de orden uno

$$
P_{i j}^{K}(\vec{x}, t)=\int m C_{i} C_{j}, f(\vec{x}, \vec{c}, t) d \vec{c},
$$

parte cinéticas del tensor de presiones, momento de orden dos

$$
S_{i j k}(\vec{x}, t)=\int m C_{i} C_{j} C_{k} f(\vec{x}, \vec{c}, t) d \vec{c},
$$

momento de orden tres, etc.

En todas estas definiciones $m$ es la masa de las partículas, y $C_{i}=c_{i}-u_{i}$ es la velocidad relativa de las partículas con respecto a la velocidad hidrodinámica y se le conoce como velocillad peculiar ó caótica.

De particular importancia son algunos momentos que se obtienen a partir de los anteriores, a través de una contracción tensorial, como por ejemplo

$$
P_{r r}(\vec{x}, t)=\int m C^{2} f(\vec{x}, \vec{c}, t) d \vec{c},
$$

la traza del tensor de presiones,

$$
S_{i j j}^{K}(\vec{x}, t)=2 \int \frac{1}{2} m C^{2} C_{i} f(\vec{x}, \vec{c}, t) d \vec{c},
$$

la parte cinética del flujo del calor. Así como otros que se obtienen al tomar la parte simétrica sin traza solamente,

$$
\stackrel{\circ}{P}_{i j}^{K}(\vec{x}, t)=P_{i j}^{K}(\vec{x}, t)-\frac{1}{3} P_{r r}^{K}(\vec{x}, t) \delta_{i j}
$$


siendo $\delta_{i j}$ el tensor unitario de segundo orden.

Una cantidad de gran importancia en la teoría cinética es la temperatura local del sistema. Para nuestro problema y debido a que las moléculas sólo tienen grados de libertad traslacionales, la definiremos en términos de la energía cinética relativa. De hecho, la energía interna $e(\vec{x}, t)$ del gas sólo es energía cinética de manera que tenemos las relaciones siguientes;

$$
\begin{aligned}
e(\vec{x}, t) & =\frac{1}{2 n(\vec{x}, t)} P_{r r}^{K}(\vec{x}, t) \\
& =\frac{3}{2} k_{B} T(\vec{x}, t)
\end{aligned}
$$

o bien

$$
\frac{1}{3} P_{r r}^{K}(\vec{x}, t)=n(\vec{x}, t) K_{B} T(\vec{x}, t)
$$

siendo $K_{B}$ la constante de Boltzmann.

Por contracción tensorial de los últimos flujos traslacionales podemos obtener explícitamente, la temperatura local,

$$
T(\vec{x}, t)=\frac{1}{3 K_{B} n(\vec{x}, t)} \int m C^{2} f(\vec{x}, \vec{c}, t) d \vec{c}
$$

así como la parte cinética (traslacional) del flujo de calor, ecuación (2.6)

$$
q_{i}^{K}(\vec{x}, t)=\int \frac{1}{2} m C^{2} C_{i} f(\vec{x}, \vec{c}, t) d \vec{c} .
$$

El punto de vista adoptado aquí, es que conviene definir ciertos momentos para obtener información sobre el comportamiento de $f$, estos momentos traslacionales están definidos en forma precisa para gases de Enskog.

A hora bien, en un gas monatómico denso formado por esferas cluras, existen dos mecanismos de transporte microscópico para las propiedades moleculares:

i) Por movimiento molecular traslacional, que contribuye a la parte cinética de los flujos $\left({ }^{K}\right)$ y que hemos definido anteriormente.

ii) Por intercambio en las colisiones moleculares que contribuye a la parte potencial de los flujos $\left({ }^{V}\right)$. 
Las contribuciones colisionales (potenciales) de los flujos macroscópicos se obtienen a partir de la ecuación (1.9), en la aproximación de segundo orden en potencias del diámetro molecular. En particular, para el flujo de ímpetu colisional, debemos hacer:

$$
\psi^{\prime}-\psi=m C_{i}^{\prime}-m C_{i}=m c_{i}^{\prime}-m c_{i},
$$

que representa el intercambio colisional de ímpetu.

Directamente de la eculación (1.9) obtenemos,

$$
P_{i j}^{V}(\vec{x}, t) \cong P_{i j}^{I}(\vec{x}, t)+P_{i j}^{I I}(\vec{x}, t)
$$

siendo,

$$
P_{i j}^{I}(\vec{x}, t)=\frac{a^{3}}{2} \chi \int m\left(C_{i}^{\prime}-C_{i}\right) f f_{1} k_{j}(\vec{g} \cdot \hat{k}) d \hat{k} d \vec{c} d \vec{c}_{1}
$$

y

$$
P_{i j}^{I I}(\vec{x}, t)=\frac{a^{4}}{4} \chi \int m\left(C_{i}^{\prime \prime}-C_{i}\right) f f_{1} \frac{\partial}{\partial x_{k}}\left(\ln \frac{f}{f_{1}}\right) k_{j} k_{k}(\vec{g} \cdot \hat{k}) d \hat{k} d \vec{c} d \vec{c}_{1} .
$$

La cantidad de mayor interés, en relación con los coeficientes de transporte generalizados es la contribución total del flujo cle ímpetu, es decir, el tensor de presiones completo:

$$
P_{i j}(\vec{x}, t)=P_{i j}^{K}(\vec{x}, t)+P_{i j}^{V}(\vec{x}, t)
$$

De la misma forma, para obtener la contribución colisional del flujo de calor, haremos

$$
\psi^{\prime}-\psi=\frac{1}{2} m C^{\prime 2}-\frac{1}{2} m C^{2}
$$

que representa el intercambio colisional de energía.

Nuevamente, a partir de la ec: (1.9) tenemos,

$$
q_{i}^{V}(\vec{x}, t) \cong q_{i}^{I}(\vec{x}, t)+q_{i}^{I I}(\vec{x}, t)
$$

donde,

y

$$
q_{i}^{I}(\vec{x}, t)=\frac{a^{3}}{2} \chi \int \frac{1}{2} m\left(C^{\prime 2}-C^{2}\right) f f_{\mathrm{l}} k_{i}(\vec{g} \cdot \hat{k}) d \hat{k} d \vec{c} d \vec{c}_{1}
$$

$$
q_{i}^{I I}(\vec{x}, t)=\frac{a^{4}}{4} \chi \int \frac{1}{2} m\left(C^{2}-C^{2}\right) f f_{1} \frac{\partial}{\partial x_{j}}\left(\ln \frac{f}{f_{1}}\right) k_{i} k_{j}(\vec{g} \cdot \hat{k}) d \hat{k} d \vec{c} d \vec{c}_{1} .
$$


También en este caso, la canticlad principal es la contribución total del flujo de energía, esto es, el flujo de calor completo:

$$
\underset{\text { total }}{q_{i}(\vec{x}, t)}=q_{i}^{K}(\vec{x}, t)+q_{i}^{V}(\vec{x}, t) .
$$

El punto de vista que hemos adoptado al elegir a las variables relevantes y con ello a los momentos de la función de distribución importantes, es que es suficiente definir y obtener información respecto al comportamiento de un cierto número de momentos para obtener una aproximación razonable en la función de distribución. Los momentos que vamos a seleccionar son cantidades que están definidas de manera precisa en la teoría de Enskog. Para empezar nos damos cuenta de que la parte cinética de los flujos definidos anteriormente, son los momentos de la función de distribución. Por otra parte, en la teoría de Enskog las partículas interactúan con potencial de esfera dura. Esto significa que el efecto de la interacción sólo es geométrico y se va a reflejar en el tamaño del volumen fase accesible a las partículas, en este caso las colisiones son instantáneas y la interacción únicamente se da por contacto (colisión) y no por dispersión causadla por un potencial. Todo esto hace pensar que las partes cinéticas de los flujos son las cantidades relevantes en la descripción.

Además podemos ver con claridad en las ecuaciones (2.14) y (2.18) que las transferencias colisionales quedan escritas en términos de cantidades dependientes de las velocidades y ciertas combinaciones de las funciones de distribución, haciendo pensar que al menos de manera aproximadla se podrán escribir en términos de los momentos cinéticos.

\subsection{La función de distribución. Aproximación a 13- momentos}

La idea principal detrás del método de momentos de Grad, es que cualquier función de distribución fuera de equilibrio se puede expresar como una serie infinita de sus momentos, tomando como base del desarrollo, una función de peso y algún conjunto completo ortonormal de funciones tensoriales de la velocidad molecular $[39,59,61]$. Por lo tanto, la función de distribución se puede expresar como:

$$
f(\vec{x}, \vec{c}, t)=f^{(0)}(\vec{x}, \vec{c}, t) \sum_{r=0}^{\infty} \frac{1}{r !} a_{i}^{(r)}(\vec{x}, t) \mathcal{H}_{i}^{(r)}(\vec{c}),
$$


donde $i=i_{1}, i_{2} \ldots$ es un índice múltiple. Esta expresión solamente es válida para gases densos de Enskog monoatómicos, en los cuales las moléculas sólo tienen grados de libertad traslacionales, de tal forma que el argumento de la función de distribución corresponde a los puntos representativos del espacio $(\vec{x}, \vec{c})$.

La función de peso alrededor de la cual se hace este desarrollo, se escoge generalmente como la función de distribución Maxwelliana local correspondiente a los invariantes colisionales del sistema, es decir:

$$
f^{(0)}(\vec{x}, \vec{c}, t)=n(\vec{x}, t)\left(\frac{m}{2 \pi k_{B} T(\vec{x}, t)}\right)^{3 / 2} \exp \left\{\frac{-m C^{2}(\vec{x}, t)}{2 k_{B} T(\vec{x}, t)}\right\}
$$

donde $C_{i}(\vec{x}, t)=c_{i}-u_{i}(\vec{x}, t)$.

En el procedimiento para obtener esta función de distribución se identifican las variables relevantes conservarlas; $n(\vec{x}, t)$ la densidad de número local, $u_{i}(\vec{x}, t)$ velocidad hidrodinámica, y $T(\vec{x}, t)$ la temperatura local. En general, estas variables deben satisfacer las relaciones siguientes:

$$
\begin{gathered}
n(\vec{x}, t)=\int f^{(0)}(\vec{x}, \vec{c}, t) d \vec{c} \\
u_{i}(\vec{x}, t)=\frac{1}{n} \int c_{i} f^{(0)}(\vec{x}, \vec{c}, t) d \vec{c} \\
\frac{3}{2} n k_{B} T(\vec{x}, t)=\int \frac{1}{2} m C^{2} f^{(0)}(\vec{x}, \vec{c}, t) d \vec{c}
\end{gathered}
$$

independientemente del estado fuera de equilibrio del sistema.

La base de funciones ortonormales que utilizamos en el desarrollo, corresponde al conjunto completo de polinomios multidimensionales de Hermite en las velocidades moleculares, $\mathcal{H}_{i}^{(r)}(\vec{c})[59,60]$. Estos polinomios son esencialmente productos de los polinomios de Hermite en una sola variable, expresados en forma simétrica y en notación invariante. La forma más directa de obtenerlos es utilizando una función generadora, que actúa como derivadas sucesivas sobre la función de peso escrita en variables adimensionales.

Explícitamente los polinomios de Hermite [59-60] son:

$$
\begin{aligned}
& \mathcal{H}^{(0)}=1 \\
& \mathcal{H}_{i}^{(1)}=v_{i} \\
& \mathcal{H}_{i j}^{(2)}=v_{i} v_{j}-\delta_{i j} \\
& \mathcal{H}_{i j k}^{(3)}=v_{i} v_{j} v_{k}-\left(v_{i} \delta_{j k}+v_{j} \delta_{k i}+v_{k} \delta_{i j}\right)
\end{aligned}
$$


donde las velocidades moleculares se hacen adimensionales por medio de la velocidad térmica;

$$
v_{i}=\frac{c_{i}}{\sqrt{\frac{k_{B} T}{m}}}
$$

Los coeficientes del clesarrollo de $f(\vec{x}, \vec{c}, t)$ se pueden obtener considerando las propiedades de simetría y ortogonadidad de los polinomios de Hermite, de donde:

$$
n(\vec{x}, t) a_{i}^{(r)}(\vec{x}, t)=\int \mathcal{H}_{i}^{(r)}(\vec{c}) f(\vec{x}, \vec{c}, t) d \vec{c}
$$

la sustitución directa de la ec. (2.25) en la ec. (2.27) nos permite identificar los momentos de desarrollo de $f$, con las variables relevantes traslacionales del gas de Enskog. En efecto, algunos valores explícitos son:

$$
\begin{aligned}
& a^{(0)}(\vec{x}, t)=1 \\
& a_{i}^{(1)}(\vec{x}, t)=0 \\
& \dot{a}_{i j}^{(2)}(\vec{x}, t)=\frac{F_{i j}^{\prime K}(\vec{x}, t)}{n k_{B} T} \\
& a_{i j j}^{(3)}(\vec{x}, t)=2 \sqrt{\frac{m}{k_{B} T}} \frac{q^{K}(\vec{x}, t)}{n k_{B} T}
\end{aligned}
$$

Notemos que de acuerdo con la ecuación (2.20), la función de distribución fuera de equilibrio puede escribirse completamente en términos de los momentos traslacionales, es decir, la parte cinética de los flujos de ímpetu y energía.

En el clesarrollo de la función de distribución se pueden utilizar directamente los momentos anteriores, así como combinaciones lineales de los mismos; en particular vamos a considerar la forma siguiente:

$$
\begin{aligned}
f(\vec{x}, \vec{c}, t)= & f^{(0)}(\vec{x}, \vec{c}, t)\left\{\frac{1}{0 !} a^{(0)}(\vec{x}, t) \mathcal{H}^{(0)}(\vec{c})+\frac{1}{1 !} a_{i}^{(1)}(\vec{x}, t) \mathcal{H}_{i}^{(1)}(\vec{c})\right. \\
& \left.+\frac{1}{2 !} a_{i j}^{(2)}(\vec{x}, t) \mathcal{H}_{i j}^{(2)}(\vec{c})+\frac{1}{3 !} a_{i j k}^{(3)}(\vec{x}, t) \mathcal{H}_{i j k}^{(3)}(\vec{c})+\cdots\right\} .
\end{aligned}
$$

Para fines prácticos, debemos cortar la serie en algún número de términos determinado; en cualquier caso los momentos de orden superior se hacen 
igual a cero. En la aproximación a 13-momentos se eligen como variables relevantes;

$$
n(\vec{x}, t), u_{i}(\vec{x}, t), P_{i j}^{K}(\vec{x}, t), q_{i}^{K}(\vec{x}, t)
$$

también, por definición de temperatura se puede expresar como:

$$
n(\vec{x}, t), u_{i}(\vec{x}, t), T(\vec{x}, t), \stackrel{P}{P}_{i j}^{K}(\vec{x}, t), q_{i}^{K}(\vec{x}, t) .
$$

Las variables conservadas se encuentran en $f^{(0)}$ mientras que los flujos de ímpetu y calor aparecerán en los términos entre las llaves de la ecuación (2.29).

De acuerdo con las propiedades de los polinomios de Hermite y considerando las definiciones de la ecuación (2.28), la aproximación a 13-momentos para la función de distribución es $[54 \mid$ $f^{(13)}(\vec{x}, \vec{c}, t)=$

$$
f^{(0)}(\vec{x}, \vec{c}, t)\left\{1+\frac{m}{2 n k_{B}^{2} T^{2}} P_{i j}^{K} C_{i} C_{j}+\frac{m}{5 n K_{B}^{2} T^{2}}\left(\frac{m C^{2}}{K_{B} T}-5\right) q_{i}^{K} C_{i}\right\},
$$

donde se sobreentiende suma sobre indices repetidos.

Esta función de distribución, aún cuando está escrita en términos de las velocidades moleculares, no tiene ningún significado con relación a la evolución del sistema, mientras no se especifiquen los momentos relevantes elegidos como funciones de la posición y del tiempo. El estado del gas se puede describir utilizando la función de distribución, o bien, en forma equivalente todos sus momentos macroscópicos. De la misma forma, la evolución temporal del estado fuera de equilibrio se puede representar por medio de la ecuación de Enskog o bien, en términos de las ecuaciones de movimiento que corresponden a las variables macroscópicas relevantes. En forma precisa, estas ecuaciones de evolución se obtienen a partir de la ecuación (1.13), lo que conduce a un conjunto de ecuaciones en derivadas parciales no-lineales. acopladas [54-56].

\subsection{Las ecuaciones de balance}

El estado macroscópico del gas de Enskog, puede caracterizarse por $13-$ momentos de Grad: la clensidad de masa $\rho(\vec{x}, t)=m n(\vec{x}, t)$, la velocidad macroscópica $u_{i}(\vec{x}, t)$, la parte cinética del tensor de presiones $P_{i j}^{K}(\vec{x}, t)$ y la 
parte cinética del flujo de calor $q_{i}^{K}(\vec{x}, t)$. Estamos interesados en obtener una teoría linealizada, conservando los flujos a segundo orden en la densidad y omitiendo los términos cuadráticos en las ecuaciones de movimiento de las variables relevantes. Las ecuaciones de balance se obtienen a partir de la ecuación (1.13) haciendo:

i) Balance de masa: $\psi=m$

$$
\frac{\partial \rho}{\partial t}+\frac{\partial}{\partial x_{i}}\left(\rho u_{i}\right)=0
$$

ii) Balance de ímpetu lineal: $\psi=m c_{i}$

$$
\frac{\partial}{\partial t}\left(\rho u_{i}\right)+\frac{\partial}{\partial x_{j}}\left(P_{i j}^{K}+P_{i j}^{I}+P_{i j}^{I I}+\rho u_{i} u_{j}\right)=0
$$

iii) Balance del tensor de presiones: $\psi=m C_{i} C_{j}$

$$
\begin{aligned}
& \frac{\partial P_{i j}^{K}}{\partial t}+\frac{\partial}{\partial x_{k}}\left(P_{i j k}^{K}+P_{i j k}^{I}+P_{i j k}^{I I}+P_{i j}^{K} u_{k}\right)+ \\
& \left(P_{i k}^{K}+P_{i k}^{I}+P_{i k}^{I I}\right)\left(\frac{\partial u_{j}}{\partial x_{k}}\right)+\left(P_{j k}^{K}+P_{j k}^{I}+P_{j k}^{I I}\right)\left(\frac{\partial u_{i}}{\partial x_{k}}\right)- \\
& -\frac{1}{2}\left\{\tilde{\Lambda}_{i k \ell}^{I I} \frac{\partial^{2} u_{j}}{\partial x_{k} \partial x_{\ell}}+\tilde{\Lambda}_{j k \ell}^{I I} \frac{\partial^{2} u_{i}}{\partial x_{k} \partial x_{\ell}}\right\}-\frac{\partial^{2} P_{i j k \ell}}{\partial x_{k} \partial x_{\ell}}=\mathbb{P}_{i j}^{K}+\mathbb{P}_{i j}^{I}+\mathbb{P}_{i j}^{I I}
\end{aligned}
$$

iv) Balance del flujo de calor $\psi=\frac{1}{2} m C^{2} C_{i}$

$$
\begin{aligned}
& \frac{\partial q_{i}^{K}}{\partial t}+\frac{\partial}{\partial x_{j}}\left(q_{i j}^{K}+q_{i j}^{I}+q_{i j}^{I I}+q_{i}^{K} u_{j}\right)+\left(q_{j}^{K}+q_{j}^{I}+q_{j}^{I I}\right)\left(\frac{\partial u_{i}}{\partial x_{j}}\right) \\
& -\frac{\partial^{2} q_{i j k}^{I I}}{\partial x_{j} \partial x_{k}}-\frac{P_{i \ell}^{K}}{\rho} \frac{\partial}{\partial x_{j}}\left(P_{\ell j}^{K}+P_{\ell j}^{I}+P_{\ell j}^{I I}\right)-\frac{1}{2} \frac{P_{r r}}{\rho} \frac{\partial}{\partial x_{j}}\left(P_{i j}^{K}+P_{i j}^{I}+P_{i j}^{I I}\right) \\
& +\left(P_{i \ell j}^{K}+P_{i \ell j}^{I}+P_{i \ell j}^{I I}\right)\left(\frac{\partial u_{\ell}}{\partial x_{j}}\right)-\frac{1}{2} P_{\ell \ell j k}^{I I}\left(\frac{\partial^{2} u_{i}}{\partial x_{j} \partial x_{k}}\right)-P_{i \ell j k}^{I I}\left(\frac{\partial^{2} u_{\ell}}{\partial x_{j} \partial x_{k}}\right) \\
& =\mathbb{Q}_{i}^{K}+\mathbb{Q}_{i}^{I}+\mathbb{Q}_{i}^{I I}
\end{aligned}
$$


En estas ecuaciones hemos introducido algunas cantidades que provienen de los flujos por colisión tales como;

$$
\begin{aligned}
& P_{i j k}^{I}=\frac{a}{2} \int \chi m\left(C_{i}^{\prime \prime} C_{j}^{\prime}-C_{i} C_{j}\right) k_{k} f f_{1} d \Gamma \\
& P_{i j k}^{I I}=\frac{a^{2}}{4} \int \chi m\left(C_{i}^{\prime} C_{j}^{\prime}-C_{i} C_{j}\right) \frac{\partial}{\partial x_{\ell}}\left(\ln \frac{f}{f_{1}}\right) k_{k} k_{\ell} f f_{1} d \Gamma \\
& \Lambda_{i j k}^{I I}=\frac{a^{2}}{4} \int \chi m\left(C_{i}^{\prime}-C_{i}\right) k_{j} k_{k} f f_{1} d \Gamma \\
& P_{i j k \ell}^{I I}=\frac{a^{2}}{4} \int \frac{\chi m}{2}\left(C_{i}^{\prime \prime} C_{j}^{\prime \prime}-C_{i} C_{j}\right) k_{k} k_{\ell} f f_{1} d \Gamma \\
& \mathbb{P}_{i j}^{K}=\int \chi m\left(C_{i}^{\prime} C_{j}^{\prime}-C_{i} C_{j}\right) f f_{1} d \Gamma \\
& \mathbb{P}_{i j}^{I}=\frac{a}{2} \int \chi m\left(C_{i}^{\prime \prime} C_{j}^{\prime \prime}-C_{i} C_{j}\right) \frac{\partial}{\partial x_{k}}\left(\ln \frac{f}{f_{1}}\right) k_{k} f f_{1} d \Gamma \\
& \mathbb{P}_{i j}^{I I}= \\
& \frac{a^{2}}{4} \int \chi m\left(C_{i}^{\prime} C_{j}^{\prime}-C_{i} C_{j}\right)\left(\frac{\partial \ln f}{\partial x_{k}}\right)\left(\frac{\partial \ln f_{1}}{\partial x_{\ell}}\right) k_{k} k_{\ell} f f_{1} d \Gamma \\
& +\frac{a^{2}}{4} \int \frac{\chi m}{2}\left(C_{1}^{\prime} C_{j}^{\prime \prime}-C_{i} C_{j}\right)\left[\frac{1}{f} \frac{\partial^{2} f}{\partial x_{k} \partial x_{\ell}}+\frac{1}{f_{1}} \frac{\partial^{2} f_{1}}{\partial x_{k} \partial x_{\ell}}\right] k_{k} k_{\ell} f f_{1} d \Gamma \\
& q_{i j}^{I}=\frac{a}{2} \int \frac{\chi m}{2}\left(C^{\prime 2} C_{i}^{\prime}-C^{2} C_{i}\right) k_{j} f f_{1} d \Gamma \\
& q_{i j}^{I I}=\frac{a^{2}}{4} \int \frac{\chi^{\prime m}}{2}\left(C^{\prime 2} C_{i}^{\prime}-C^{2} C_{i}\right) \frac{\partial}{\partial x_{k}}\left(\ln \frac{f}{f_{1}}\right) k_{j} k_{k} f f_{1} d \Gamma \\
& q_{i j k}^{I I}=\frac{a^{2}}{4} \int \frac{\chi^{m}}{4}\left(C^{\prime 2} C_{i}^{\prime \prime}-C^{2} C_{i}\right) k_{j} k_{k} f f_{1} d \Gamma \\
& \mathbb{Q}_{i}^{K}=\int \frac{\chi m}{2}\left(C^{\prime 2} C_{i}^{\prime}-C^{2} C_{i}\right) f f_{1} d \Gamma \\
& \mathbb{Q}_{i}^{I}=\frac{a}{2} \int \frac{\chi m}{2}\left(C^{\prime 2} C_{i}^{\prime}-C^{2} C_{i}\right) \frac{\partial}{\partial x_{j}}\left(\ln \frac{f}{f_{1}}\right) k_{j} f f_{1} d \Gamma
\end{aligned}
$$




$$
\begin{aligned}
\mathbb{Q}_{i}^{I I} & =\frac{a^{2}}{4} \int \frac{\chi m}{2}\left(C^{2} C_{i}^{\prime}-C^{2} C_{i}\right)\left(\frac{\partial \ln f}{\partial x_{j}}\right)\left(\frac{\partial \ln f_{1}}{\partial x_{k}}\right) k_{j} k_{k} f f_{1} d \Gamma \\
& +\frac{a^{2}}{4} \int \frac{\chi m}{4}\left(C^{\prime 2} C_{i}^{\prime}-C^{\prime 2} C_{i}^{\prime}\right)\left[\frac{1}{f} \frac{\partial^{2} f}{\partial x_{j} \partial x_{k}}+\frac{1}{f_{1}} \frac{\partial^{2} f_{1}}{\partial x_{j} \partial x_{k}}\right] k_{j} k_{k} f f_{1} d \Gamma
\end{aligned}
$$

donde,

$$
d \Gamma=a^{2}(\vec{g} \cdot \hat{k}) d \hat{k} d \vec{c} d \vec{c}_{1} .
$$

Los momentos cinéticos de orden superior al segundo que aparecen en estas expresiones están definidos por,

$$
\begin{aligned}
& P_{i j k}^{K}=\int m C_{i} C_{j} C_{k} f d \vec{c} \\
& q_{i j}^{K}=\int \frac{m}{2} C^{2} C_{i} C_{j} f d \vec{c} .
\end{aligned}
$$

Las ecuaciones de movimiento tienen todavía una forma muy general, sin embargo, podemos identificar a $P_{i j k}^{K}$ y $P_{i j k}^{I}, P_{i j k}^{I I}$ como las partes cinética y potencial del flujo del tensor de presiones, respectivamente. También $q_{i j}^{K}$ y $q_{i j}^{I}, q_{i j}^{I I}$ son las partes cinética y potencial correspondientes a el flujo del flujo de calor $\mathbb{P}_{i j}^{K}, \mathbb{P}_{i j}^{I}, \mathbb{P}_{i j}^{I I}, \mathbb{Q}_{i}^{K}, \mathbb{Q}_{i}^{I}$ y $\mathbb{Q}_{i}^{I I}$ son los términos de producción. Debemos notar que $P_{i j}^{I}, P_{i j}^{I I}, q_{i}^{I}, q_{i}^{I I}$ son los flujos colisionales del tensor de presiones y del flujo de calor, que ya hemos cliscutido en el capítulo anterior.

Todas las cantidades anteriores, se denominan "Integrales constitutivas", en virtud de que admiten una representación en la descripción de 13-momentos, ello en términos de las variables relevantes elegidlas y probablemente de sus derivadas hasta segundo orden. Para evaluar estas cantidades necesitamos la función de distribución correspondiente, lo que nos conduce a un conjunto cerrado de ecuaciones y linealizado para las variables relevantes del sistema.

\subsection{Integrales Constitutivas}

Para obtener una representación en la aproximación de 13-momentos para los momentos cinéticos de orden superior y de los flujos colisionales, en términos de las variables relevantes necesitamos la función de distribución de partícula simple en la misma aproximación. La sustitución directa de la ecuación (2.31) en las integrales constitutivas nos conduce a las relaciones siguientes: 
i) Flujos cinéticos linealizados de orden superior,

$$
\begin{gathered}
P_{i j k}^{K}=\frac{2}{5}\left(q_{i}^{K} \delta_{j k}+q_{j}^{K} \delta_{i k}+q_{k}^{K} \delta_{i j}\right) \\
q_{i j}^{K}=\frac{5}{2} \frac{k_{B} T}{m}\left(\frac{k_{B} T}{m} \rho \delta_{i j}+\frac{7}{5} \dot{P}_{i j}^{K}\right) .
\end{gathered}
$$

ii) Flujos colisionales linealizados y términos de producción,

$$
\begin{aligned}
& P_{i j k}^{I}=\frac{9}{25}\left(q_{j}^{K} \delta_{i k}+q_{i}^{K} \delta_{j k}+\frac{4}{9} q_{k}^{K} \delta_{i j}\right) \chi b \rho \\
& P_{i j k}^{I I}=-\tilde{\omega} \frac{k_{B}}{m}\left\{\frac{6}{5}\left(\frac{\partial T}{\partial x_{i}} \delta_{j k}\right)^{0}+\frac{\partial T}{\partial x_{k}} \delta_{i j}\right\}- \\
& -\frac{\tilde{\omega}}{2 n m}\left\{\frac{6}{5}\left(\frac{\partial \stackrel{\circ}{P}_{k j}^{K}}{\partial x_{i}}+\delta_{i k} \frac{\partial \dot{\circ}_{\ell j}^{K}}{\partial x_{\ell}}\right)^{0}+\frac{6}{5} \frac{\partial \stackrel{\circ}{P}_{i j}^{K}}{\partial x_{k}}+\frac{4}{5} \frac{\partial \dot{P}_{\ell k}}{\partial x_{\ell}} \delta_{i j}\right\} \\
& \tilde{\Lambda}_{i j k}^{I I}=0 \\
& P_{i j k \ell}^{I I}=\frac{-3 \tilde{\omega}}{140 \mathrm{~mm}}\left\{10 \stackrel{\circ}{P}_{i j}^{K} \delta_{k \ell}+3\left(\stackrel{\stackrel{P}{P}}{i k}_{j \ell}+\stackrel{\circ}{P}_{i \ell}^{K} \delta_{j k}+\stackrel{\circ}{P}_{k j}^{K} \delta_{i \ell} \stackrel{\circ}{P}_{\ell j}^{K} \delta_{i k}\right)-12 \stackrel{\circ}{P}_{k \ell}^{K} \delta_{i j}\right\} \\
& \mathbb{P}_{i j}^{K}=\frac{16}{5} A \chi \rho T^{1 / 2} \stackrel{\circ}{P}_{i j}^{K} \\
& \mathbb{P}_{i j}^{I}=\frac{6}{5}\left(\frac{k_{B} T}{m} \rho\left(\frac{\partial u_{i}}{\partial x_{j}}\right)^{0}+\frac{1}{5}\left(\frac{\partial q_{i}^{K}}{\partial x_{j}}\right)^{0}\right) \chi b \rho \\
& \mathbb{P}_{i j}^{I I}=\frac{-3 \tilde{\omega}}{14 n m}\left\{\frac{6}{5}\left(\frac{\partial^{2} \stackrel{\circ}{P}_{k_{i}}^{K}}{\partial x_{k} \partial x_{j}}\right)^{0}+\frac{\partial^{2} \stackrel{\circ}{P}_{i j}^{K}}{\partial x_{k} \partial x_{k}}\right\} \\
& q_{i j}^{I}=\frac{5}{2} \frac{k_{B} T}{m}\left(\frac{k_{B} T}{m} \rho \delta_{i j}+\frac{17}{25} \dot{P}_{i j}^{K}\right) \chi b \rho \\
& q_{i j}^{I l}=-\frac{11 \tilde{\omega}}{4} \frac{k_{B} T}{m}\left\{\frac{6}{5}\left(\frac{\partial u_{i}}{\partial x_{j}}\right)^{0}+\frac{\partial u_{k}}{\partial x_{k}} \delta_{i j}\right\}- \\
& -\frac{\tilde{\omega}}{2 n m}\left\{\frac{3}{5} \frac{\partial q_{i}^{K}}{2 x_{j}}+\frac{41}{20}\left[\frac{6}{5}\left(\frac{\partial q_{i}^{K}}{\partial x_{j}}\right)^{0}+\frac{\partial q_{k}^{K}}{\partial x_{k}} \delta_{i j}\right]\right\}
\end{aligned}
$$




$$
\begin{gathered}
q_{i j k}^{I I}=\frac{-3 \tilde{\omega}}{100 m m}\left\{5 q_{i}^{K} \delta_{j k}+\left(q_{i}^{K} \delta_{j k}+q_{j}^{K} \delta_{i k}+q_{k}^{K} \delta_{i j}\right)\right\} \\
\mathbb{Q}_{i}^{K}=-\frac{32}{15} A \chi \rho T^{1 / 2} q_{i}^{K} \\
\mathbb{Q}_{i}^{I}=\frac{k_{B} T}{m}\left(\frac{k_{B}}{m} \rho \frac{\partial T}{\partial x_{i}}+\frac{1}{10} \frac{\partial \dot{P}_{i j}^{K}}{\partial x_{j}}\right) \chi b \rho \\
\mathbb{Q}_{i}^{I I}=\frac{-3 \tilde{\omega}}{50 n m}\left\{\frac{\partial^{2} q_{k}^{K}}{\partial x_{i} \partial x_{k}}+3 \frac{\partial^{2} q_{i}^{K}}{\partial x_{k} \partial x_{k}}\right\} .
\end{gathered}
$$

En las expresiones anteriores, el superíndice ()$^{0}$ significa tomar la parte simétrica sin traza respecto de los índices $i, j$ únicamente. En los flujos colisionales de segundo orden aparece la viscocidad volumétrica del régimen hidrodinámico; que está definidla como [44]:

$$
\tilde{\omega}=\frac{4}{9} \chi a^{4} n^{2} \pi^{1 / 2}\left(m k_{B} T\right)^{1 / 2} .
$$

En los términos de producción aparece la integral de colisión de esfera dura usual $A$, así como el factor de volumen excluído:

$$
A=\left(\frac{a^{4} \pi k_{B}}{m^{3}}\right)^{1 / 2} \quad b=\frac{2}{3} \pi a^{3} / m .
$$

En el apéndice B se dan algunos ejemplos del cálculo de estas integrales constitutivas, ya que el desarrollo algebraico involucrado necesita de un tratamiento detallado de las integrales. Notemos que las colisiones no localizadas dan origen a contribuciones por transferencia colisional no solo en los flujos, sino también en los términos de producción.

En particular, la sustitución de la función de distribución, nos permite evaluar directamente los flujos colisionales de momento y energía. En efecto, sustituyendo $f^{(13)}(\vec{x}, \vec{c}, t)$ en las ecuaciones (2.14) y (2.18) obtenemos:

$$
\begin{gathered}
P_{i j}^{I}=\left(\frac{k_{B} T}{m} \rho \delta_{i j}+\frac{2}{5} \stackrel{P}{i}_{i j}^{K}\right) \chi b \rho \\
P_{i j}^{I I}=-\tilde{\omega}\left\{\frac{6}{5}\left(\frac{\partial u_{i}}{\partial x_{j}}\right)^{0}+\frac{\partial u_{k}}{\partial x_{k}} \delta_{i j}\right\}- \\
-\frac{\tilde{\omega}}{10 n m} \frac{m}{k_{B} T}\left\{\frac{6}{5}\left(\frac{\partial q_{i}^{K}}{\partial x_{j}}\right)^{0}+\frac{\partial q_{k}^{K}}{\partial x_{k}} \delta_{i j}\right\}
\end{gathered}
$$




$$
\begin{gathered}
q_{i}^{I}=\frac{3}{5} q_{i}^{K} \chi b \rho \\
q_{i}^{I I}=-c_{v} \tilde{\omega} \frac{\partial T}{\partial x_{i}}-\frac{\tilde{\omega}}{2 n m}\left\{\frac{6}{5} \frac{\partial \stackrel{\circ}{P}_{r i}^{K}}{\partial x_{r}}\right\},
\end{gathered}
$$

donde $c_{v}=\frac{3 k_{B}}{2 m}$ es la capacidad calorífica del gas [44]. Estas expresiones pueden utilizarse directamente para evaluar el flujo total de ímpetu y energía cuando se tomen en cuenta las contribuciones completas del tensor de presiones y del flujo del calor. Como ya hemos mencionado, estas cantidades tienen una parte cinética y otra colisional, las cuales sólo requieren de $f^{(13)}(\vec{x}, \vec{c}, t)$ para evaluarse completamente.

Los flujos colisionales se pueden evaluar satisfactoriamente, debido a que el gas está formado por esferas duras, prácticamente sin importar el régimen de vectores de onda. Estas cantidades están restringidas por el hecho de que el kernel colisional de Ensliog está describiendo al sistema, lo que significa que sabemos los detalles de la colisión binaria, la sección de dispersión se puede calcular explícitamente, y por lo tanto las integrales correspondientes admiten un desarrollo completo.

Notemos que las integrales constitutivas están expresadas en términos de las variables relevantes traslacionales, mismas que aparecen en el desarrollo polinomial de la función de distribución. El tensor de presiones y el flujo de calor tienen un carácter fundamental en el método de Grad; obedecen una ecuación de balance específica y están en la misma categoría que las variables conservadas.

\subsection{Ecuaciones de movimiento linealizadas}

Para resolver la ecuación de Enskog, es necesario que la función de distribución $f^{(13)}(\vec{x}, \vec{c}, t)$ tenga un comportamiento temporal adecuado. Esto es posible mediante la construcción de las ecuaciones de movimiento para las variables relevantes no-conservadias.

En el procedimiento para obtener las ecuaciones de balance, nos damos cuenta que el término de arrastre de la ecuación de Enskog, y parte del término colisional, introducen derivadas espaciales y temporales de los momentos de orden superior. En efecto, nos encontramos con una jerarquía de ecuaciones cuyos primeros elementos son las ecuaciones $(2.32)-(2.35)$, en ella 
es necesario incorporar alguna hipótesis de cerradura, la cual en este caso nos restringe a una aproximación de 13-momentos. Esta es la razón por la cual las integrales constitutivas han sido calculadas con la función de distribución $f^{(13)}(\vec{x}, \vec{c}, t)$ dando expresiones restringidlas, en el número de momentos, que se pueden expresar en términos de las variables relevantes. Para obtener explícitamente las ecuaciones de movimiento, sustituimos las integrales constitutivas de la ecuación (2.51) en la ecuación (2.64), en las ecuaciones de balance generales de las variables relevantes, omitiendo productos de los flujos de masa, ímpetu y energía, dados por: $u_{i}, \stackrel{\circ}{P_{i j}^{K}}, q_{i}^{K}$; así, como de gradientes de densidad y temperatura $\frac{\partial \rho}{\partial x_{i}}, \quad \frac{\partial T}{\partial x_{i}}$. Por lo tanto, el método de momentos de Grad nos conduce a un conjunto de ecuaciones acopladas linealizadas para las variables relevantes: las variables conservadas, $\rho, u_{i}, T$ y las variables no conservadas $\stackrel{\circ}{P}_{i j}^{K}$ y $q_{i}^{K}$ todlas ellas funciones locales del tiempo.

Las primeras conducen a las ecuaciones de conservación:

$$
\begin{gathered}
\frac{\partial \rho}{\partial t}+\rho \frac{\partial u_{i}}{\partial x_{i}}=0 \\
\rho \frac{\partial u_{i}}{\partial t}+\frac{\chi^{(\rho)}}{\alpha} \frac{\partial \rho}{\partial x_{i}}-\rho \tilde{\omega}_{0}\left\{\frac{1}{2} \frac{\partial^{2} u_{i}}{\partial x_{k} \partial x_{k}}+\frac{\partial^{2} u_{k}}{\partial x_{k} \partial x_{i}}\right\}+ \\
+\frac{\rho}{\alpha T} \chi^{(5)} \frac{\partial T}{\partial x_{i}}+\chi^{(2)} \frac{\partial \dot{P}_{i j}^{K}}{\partial x_{j}}-\frac{\alpha \tilde{\omega}}{10}\left\{\frac{1}{2} \frac{\partial^{2} q_{i}^{K}}{\partial x_{k} \partial x_{k}}+\frac{\partial^{2} q_{k}^{K}}{\partial x_{k} \partial x_{i}}\right\}=0 \\
\frac{3}{2} \frac{\rho}{\alpha T} \frac{\partial T}{\partial t}+\chi^{(3)} \frac{\partial q_{k}^{K}}{\partial x_{k}}-c_{\eta} \tilde{\omega} \frac{\partial^{2} T}{\partial x_{k} \partial x_{k}}-\frac{1}{2} \tilde{\omega}_{0} \frac{\partial^{2} \stackrel{\rho}{P}_{k \ell}^{K}}{\partial x_{k} \partial x_{\ell}}+ \\
+\frac{\rho}{\alpha} \chi^{(5)} \frac{\partial u_{k}}{\partial x_{k}}=0 .
\end{gathered}
$$

Las otras conducen a las ecuaciones de relajamiento:

$$
\begin{aligned}
& \frac{\partial \stackrel{\circ}{P}_{i j}^{K}}{\partial t}-\frac{4}{7} \tilde{\omega}_{0}\left(\frac{\partial^{2} \stackrel{o}{P}_{k i}^{K}}{\partial x_{k} \partial x_{j}}\right)^{0}-\frac{1}{7} \tilde{\omega}_{0} \frac{\partial^{2} \stackrel{\circ}{P}_{i j}^{K}}{\partial x_{k} \partial x_{k}}+2 \chi^{(2)} \frac{\rho}{\alpha}\left(\frac{\partial u_{i}}{\partial x_{j}}\right)^{0}+ \\
& \frac{4}{5} \chi^{(3)}\left(\frac{\partial q_{i}^{K}}{\partial x_{j}}\right)^{0}-\frac{4}{5} c_{v} \tilde{\omega}\left(\frac{\partial^{2} T}{\partial x_{j} \partial x_{i}}\right)^{0}=-\frac{1}{\tau_{p}} \stackrel{\rho}{P}_{i j}^{K}
\end{aligned}
$$




$$
\begin{aligned}
& \frac{\partial q_{i}^{K}}{\partial t}-\frac{2}{3} \tilde{\omega}_{0} \frac{\partial^{2} q_{k}^{K}}{\partial x_{k} \partial x_{i}}-\frac{1}{3} \tilde{\omega}_{0} \frac{\partial^{2} q_{i}^{K}}{\partial x_{k} \partial x_{k}}+\frac{5 \rho}{2 \alpha^{2} T} \chi^{(3)} \frac{\partial T}{\partial x_{i}}+ \\
& +\frac{\chi^{(3)}}{\alpha} \frac{\partial \dot{P}_{i j}^{K}}{\partial x_{j}}-\frac{1}{8} \frac{\rho}{\alpha} \tilde{\omega}_{0} \frac{\partial^{2} u_{i}}{\partial x_{k} \partial x_{k}}-\frac{1}{4} \frac{\rho}{\alpha} \tilde{\omega}_{0} \frac{\partial^{2} u_{k}}{\partial x_{k} \partial x_{i}}= \\
& =-\frac{1}{\tau_{q}} q_{i}^{K} .
\end{aligned}
$$

Para simplificar las expresiones, hemos considerado las definiciones siguientes;

$$
\begin{gathered}
\chi^{(\nu)}=\left(1+\frac{\nu}{5} \chi b \rho\right) ; \quad \nu=1,2,3 \ldots \\
\chi^{(\rho)}=\left(1+2 \chi b \rho+\frac{\partial \chi}{\partial \rho} b \rho^{2}\right) ; \\
\tilde{\omega}_{0}=\frac{6 \tilde{\omega}}{5 n m}, \quad \alpha=\frac{m}{k_{B} T}, \quad c_{v}=\frac{3 k_{B}}{2 m} \\
\tau_{p}^{-1}=\frac{16}{5} A \rho T^{1 / 2} \chi^{-1} \\
\tau_{q}^{-1}=\frac{32}{15} A \rho T^{1 / 2} \chi^{-1} \\
\tau_{p}=\frac{2}{3} \tau_{q} .
\end{gathered}
$$

Denominaremos a $\chi^{(\nu)}, \chi^{(\rho)}$ los factores de correlación de la densidad, $\tilde{\omega}_{0}$ es proporcional a una viscocidad volumétrica cinemática, $\alpha$ es el inverso del cuadrado de la velocidad térmica, mientras que $\tau_{p}, \tau_{q}$ son los tiempos de relajación de las variables no-conservadas, que son del orden de los correspondientes a gases diluidos en el régimen de densidades moderadas.

La característica principal del método de Grad en la aproximación de 13-momentos consiste en que el tensor de esfuerzos y el flujo de calor son variables fundamentales con su propia ecuación de movimiento; ello en contraste con el esquema de la hidrodinámica usual donde ellas se introducen por medio de ecuaciones constitutivas. Los tiempos de relajación asociados con estas cantidades se expresan directamente en términos de las integrales colisionales moleculares y como veremos determinan directamente a los coeficientes de transporte en un intervalo amplio de la región $(\vec{k}, \omega)$ finita. En lo 
que sigue nos referiremos a las ecuaciones (2.71)-(2.75) como las ecuaciones de Grad en 13-momentos. Estas ecuaciones se reducen a las ecuaciones del gas diluido en el régimen de bajas densidades, donde los flujos colisionales tienen una contribución despreciable y por lo tanto, no se necesita tomar en cuenta correlaciones espaciales.

Las ecuaciones de movimiento se pueden separar directamente en los flujos colisionales a primer y segundo orden, constituyendo una generalización de la descripción de Navier-Stokes de la hidrodinámica usual que nos proporciona un medio para tratar el comportamiento del sistema fuera del régimen hidrodinámico, esto es, en la región de la hidrodinámica generalizada. 


\section{Capítulo 3}

\section{Coeficientes de Transporte Generalizados}

\section{Primer Orden en los flujos Colisionales}

Para obtener los coeficientes de transporte en la región de $(\vec{k}, \omega)$ finito, utilizamos la forma linealizadla de las ecuaciones de movimiento (2.71)-(2.75) en el espacio de Fourier. Al resolverlas para la parte cinética del tensor de presiones y del flujo de calor en términos de las variables conservadas nos encontramos con relaciones constitutivas del tipo Navier-Newton-Fourier donde los coeficientes de transporte son funciones del vector de onda y la frecuencia $[38-39,62]$.

Los flujos completos se obtienen incorporando contribuciones a los flujos por transferencia colisional y para empezar solamente vamos a considerar los términos a primer orden en los gradientes, que como veremos constituyen la contribución más importante en densidades moderadas.

\subsection{Ecuaciones de movimiento en el espacio $(\vec{k}, \omega)$}

Las ecuaciones de movimiento para las variables relevantes tienen una solución estacionaria para el estado de equilibrio termodinámico, donde el sistema se encuentra en reposo y no existen procesos de relajación. En el esquema de los 13-momentos que estamos considerando, el estado estacionario asociado al equilibrio termodinámico corresponde a las condiciones siguientes;

$$
\rho=\rho_{0}, \quad T=T_{0}, \quad \vec{q}=0, \quad P=p_{0}, \quad P_{i j}=0 .
$$

Si ahora tomamos a dicho estado como referencia y queremos estudiar las fluctuaciones alrededor de él, o bien provocamos una perturbación ligera de manera que las variables relevantes no se anulen, podremos estudiar el comportamiento de nuestro sistema utilizando el conjunto de ecuaciones que construimos en el capítulo anterior linealizándolas alrededor de la solución correspondiente al estado de equilibrio.

La forma linealizada de las ecuaciones de movimiento que solo considera 
flujos colisionales de primer orden es,

$$
\begin{gathered}
\frac{\partial \rho^{\prime}}{\partial t}+\rho \frac{\partial u_{i}^{\prime}}{\partial x_{i}}=0 \\
\rho \frac{\partial u_{i}}{\partial t}+\frac{\chi^{(\rho)}}{\alpha} \frac{\partial \rho^{\prime}}{\partial x_{i}}+\frac{\rho}{\alpha T} \chi^{(5)} \frac{\partial T^{\prime}}{\partial x_{i}}+\chi^{(2)} \frac{\partial \dot{P}_{i j}^{K^{\prime}}}{\partial x_{j}}=0 \\
\frac{3}{2} \frac{\rho}{\alpha T} \frac{\partial T^{\prime}}{\partial t}+\chi^{(3)} \frac{\partial q_{k}^{K^{\prime}}}{\partial x_{k}}+\frac{\rho}{\alpha} \chi^{(5)} \frac{\partial u_{k}^{\prime}}{\partial x_{k}}=0 \\
\frac{\partial \dot{P}_{i j}^{K^{\prime \prime}}}{\partial t}+2 \chi^{(2)} \frac{\rho}{\alpha}\left(\frac{\partial u_{i}^{\prime}}{\partial x_{j}}\right)^{0}+\frac{4}{5} \chi^{(3)}\left(\frac{\partial q_{i}^{K^{\prime}}}{\partial x_{j}}\right)^{0}=-\frac{1}{\tau_{p}} \dot{\rho}_{i j}^{K^{\prime \prime}}
\end{gathered}
$$

$\mathrm{y}$

$$
\frac{\partial q_{i}^{K^{\prime}}}{\partial t}+\frac{5 \rho}{2 \alpha^{2} T} \chi^{(3)} \frac{\partial T^{\prime}}{\partial x_{i}}+\frac{\chi^{(3)}}{\alpha} \frac{\partial \stackrel{o}{P}_{i j}^{K^{\prime}}}{\partial x_{j}}=-\frac{1}{\tau_{q}} q_{i}^{K^{\prime}}
$$

Las cantidades sin prima están evaluadas en equilibrio, mientras que las cantidades primadias son las desviaciones que dependen de la posición y del tiempo, es decir, son variables locales. Las ecuaciones (3.1)-(3.5) son ecuaciones hidrodinámicas de las fluctuaciones alrededor del equilibrio termodinámico dentro del esquema linealizado obtenido a partir de la Teoría de Enskog. Suponemos que las fluctuaciones son de amplitud pequeña y no son capaces de generar inhomogeneidades grandes aún cuando pueden describir procesos rápidos a distancias muy pequeñas.

Las primeras ecuaciones (3.1)-(3.3) corresponden a las ecuaciones de conservación para las variables $\rho^{\prime}, u_{i}^{\prime}, T^{\prime}$. Las últimas ecuaciones (3.4)-(3.5) son las ecuaciones de relajamiento para las variables no conservadas $\stackrel{\circ}{P}_{i j}^{K^{\prime}}$, $q_{i}^{K^{\prime}}$. Con el objeto de estudiar las características de estas ecuaciones, las escribimos en la forma siguiente:

$$
\begin{gathered}
\frac{\partial}{\partial t} \dot{P}_{i j}^{K^{\prime \prime}}=-\frac{1}{\tau_{p}}\left[\dot{P}_{i j}^{K^{\prime \prime}}+2 \eta_{0} \frac{\chi^{(2)}}{\chi}\left(\frac{\partial u_{i}^{\prime}}{\partial x_{j}}\right)^{0}\right]-\frac{4}{5} \chi^{(3)}\left(\frac{\partial q_{i}^{K^{\prime \prime}}}{\partial x_{j}}\right)^{0} \\
\frac{\partial q_{i}^{K^{\prime \prime}}}{\partial t}=-\frac{1}{\tau_{q}}\left[q_{i}^{K^{\prime \prime}}+\lambda_{0} \frac{\chi^{(3)}}{\chi} \frac{\partial T^{\prime}}{\partial x_{i}}\right]-\frac{\chi^{(3)}}{\alpha} \frac{\partial \stackrel{\circ}{P}_{i j}^{K^{\prime \prime}}}{\partial x_{j}}
\end{gathered}
$$


aquí, hemos introducido los coeficientes de transporte usuales para el gas diluido en el régimen hidrodinámico, que están definidos como [63]:

$$
\eta_{0}=n k_{B} T \tau_{p} \chi, \quad \lambda_{0}=\frac{5 n k_{B}^{2} T}{2 m} \tau_{q} \chi
$$

Recordemos que $\eta_{0}$ es la viscosidad cortante y $\lambda_{0}$ es la conductividad térmica, que por ser cantidades propias del gas diluido, son independientes de la densidad. Si tomamos en cuenta la expresión para los tiempos de relajamiento propios de un gas de esferas duras, observamos que se cumplen las relaciones siguientes:

$$
\eta_{0}=\frac{4 m}{15 k_{B}} \lambda_{0}, \quad \tau_{p}=\frac{2}{3} \tau_{q} .
$$

Es importante notar que en las ecuaciones (3.6)-(3.7) intervienen los tiempos de relajamiento en una forma tal que si consideramos tiempos $t>>\tau_{p}, \tau_{q}$ se recuperan las ecuaciones constitutivas de Navier-Newton y Fourier. Todo ello con los coeficientes de transporte modificados por la dependencia en la densidad que está representada por las funciones $\chi$ 's. Cuando se trabaja a tiempos cortos, $t \simeq \tau_{p} \tau_{q}$ dichas ecuaciones corresponden al relajamiento de los flujos correspondientes.

Ahora bien, la distribución en longitudes de onda y frecuencia para las fluctuaciones, se observa con más claridad en el espacio de Fourier. Tomando la transformadia correspondiente al espacio $(\vec{k}, \omega)$ y denotándola con tilde $(\tilde{})$ se obtiene:

$$
\begin{gathered}
-i \omega \tilde{\rho}+\rho i k_{i} \tilde{u}_{i}=0 \\
-i \omega \rho \tilde{u}_{i}+\frac{\chi^{(p)}}{\alpha} i k_{i} \tilde{\rho}+\frac{\rho}{\alpha T} \chi^{(5)} i k_{i} \tilde{T}+\chi^{(2)} i k_{j} \tilde{P}_{i j}^{K}=0 \\
-i \omega \frac{3}{2} \frac{\rho}{\alpha T} \tilde{T}+\chi^{(3)} i k_{k} \tilde{q}_{k}^{K}+\frac{\rho}{\alpha} \chi^{(5)} i k_{k} \tilde{u}_{k}=0 \\
\left(1-i \omega \tau_{p}\right) \tilde{\tilde{P}}_{i j}^{K}+2 \eta_{0} \frac{\chi^{(2)}}{\chi}\left(i k_{j} \tilde{u}_{i}\right)^{0}+\frac{4}{5} \tau_{p} \chi^{(3)}\left(i k_{j} \tilde{q}_{i}^{K}\right)^{0}=0 \\
\left(1-i \omega \tau_{q}\right) \tilde{q}_{i}^{K}+\lambda_{0} \frac{\chi^{(3)}}{\chi} i k_{i} \tilde{T}+\frac{\tau_{q}}{\alpha} \chi^{(3)} i k_{j} \tilde{\tilde{P}}_{i j}^{K}=0 .
\end{gathered}
$$

Estas ecuaciones constituyen uno de los principales resultados del método de Grad, en relación con el comportamiento hidrodinámico generalizado del gas. Las ecuaciones del flujo de calor y del tensor de presiones son ecuaciones 
de relajación inhomogéneas, que se pueden simplificar de acuerdo con alguna situación experimental específica. Un estudio de los estados estacionarios correspondientes nos conduce a valores numéricos de los tiempos de relajación, así como a las relaciones constitutivas usuales del régimen hidrodinámico.

En hidrodinámica generalizada es usual estudiar por separado el comportamiento longitudinal y tansversal de las ecuaciones de movimiento. Las ecuaciones (3.10)-(3.14) se utilizarán para determinar dicho comportamiento para las fluctuaciones en un gas denso. En un fluido isótropo las formas invariantes en el espacio $\vec{k}$, que hacen posible dicha separación, son los operadores de proyección:

$$
k^{2} \mathbb{L}=\vec{k} \vec{k} \quad k^{2} \mathbb{Q}_{t}=k^{2} I I-\vec{k} \vec{k},
$$

donde $I I=\delta_{i j}$ es el tensor uniclad.

El operador $\mathbb{L}$ - separa la componente de las ecuaciones de movimiento en la dirección del vector de onda, mientras que $\mathbb{Q}_{t} \cdot$ proyecta sobre el espacio ortogonal a esta dirección.

Bajo estas condiciones y con ayuda de los operadores de proyección obtenemos para la parte longitudinal de las ecuaciones de conservación el conjunto siguiente:

$$
\begin{gathered}
-i \omega \tilde{\rho}+\rho\left(i k_{i} \tilde{u}_{i}\right)=0 \\
-i \omega \rho i k_{i} \tilde{u}_{i}+\frac{\chi^{(\rho)}}{\alpha}\left(i k_{i} i k_{i}\right) \tilde{\rho}+\frac{\rho}{\alpha T} \chi^{(5)}\left(i k_{i} i k_{i}\right) \tilde{T}+\chi^{(2)}\left(i k_{i} i k_{j}\right) \tilde{\stackrel{P}{P}}_{i j}^{K}=0 \\
-i \omega \frac{3}{2} \frac{\rho}{\alpha T} \tilde{T}+\chi^{(3)} i k_{i} \tilde{q}_{i}^{K}+\frac{\rho}{\alpha} \chi^{(5)} i k_{i} \tilde{u}_{i}=0
\end{gathered}
$$

y para la parte transversal de las ecuaciones de conservación

$$
-i \omega \rho \tilde{u}_{i_{t}}+\chi^{(2)} \mathbb{Q}_{t} \cdot\left(i k_{j} \stackrel{\stackrel{\circ}{P}}{K}_{i j}^{K}\right)=0 .
$$

En cuanto a la parte longitudinal de las ecuaciones de relajamiento tenemos:

$$
\begin{gathered}
\left(1-i \omega \tau_{p}\right) i k_{i} i k_{j} \stackrel{\tilde{P}}{P}_{i j}^{K}-\frac{4}{3} \eta_{0} \frac{\chi^{(2)}}{\chi} k^{2}\left(i k_{i} \tilde{u}_{i}\right)-\frac{8}{15} \tau_{p} \chi^{(3)} k^{2}\left(i k_{i} \tilde{q}_{i}^{K}\right)=0, \\
\left(1-i \omega \tau_{q}\right) i k_{i} \tilde{q}_{i}^{K}-\lambda_{0} \frac{\chi^{(3)}}{\chi} k^{2} \tilde{T}+\frac{\tau_{q}}{\alpha} \chi^{(3)}\left(i k_{i} i k_{j} \stackrel{\stackrel{o}{P}}{P}_{i j}^{K}\right)=0,
\end{gathered}
$$

mientras que para la parte transversal de las ecuaciones de relajamiento:

$$
\left(1-i \omega \tau_{p}\right) \mathbb{Q}_{t} \cdot\left(i k_{j} \tilde{\tilde{P}}_{i j}^{K}\right)-\eta_{0} \frac{\chi^{(2)}}{\chi} k^{2} \tilde{u}_{i_{t}}-\frac{2}{5} \tau_{p} \chi^{(3)} k^{2} \tilde{q}_{i}^{K}=0
$$




$$
\left(1-i \omega \tau_{q}\right) \tilde{q}_{i_{t}}^{K}+\frac{\tau_{q}}{\alpha} \chi^{(3)} \mathbb{Q}_{t} \cdot\left(i k_{j} \tilde{\stackrel{\rho}{P}}_{i j}^{K}\right)=0
$$

notemos que todas las variables relevantes con tilde corresponden a transformaciones de Fourier siendo funciones del vector de onda $\vec{k}$ y la frecuencia $\omega$.

De esta forma las ecuaciones de movimiento constituyen un sistema algebraico acoplado para las variables relevantes. Esto nos permite obtener información sobre las relaciones constitutivas que se requieren en la cerradura de las ecuaciones de balance.

\subsection{Ecuaciones constitutivas generalizadas}

Para obtener una descripción hidrodinámica del gas clenso en el espacio de variables conservadas $\tilde{\rho}, \tilde{u}_{i}, \tilde{T}$, necesitamos resolver las ecuaciones de movimiento del tensor de presiones $\tilde{\stackrel{P}{P}}_{i j}^{K}$ y del flujo del calor $\tilde{q}_{i}^{K}$. Este procedimiento consiste en desacoplar las ecuaciones (3.20)-(3.23) en la forma de ecuaciones constitutivas generalizadias, que podemos expresar como:

$$
\begin{gathered}
i k_{i} \tilde{q}_{i}^{K}=\Lambda^{K}(\vec{k}, \omega) k^{2} \tilde{T}-\Gamma_{1}^{K}(\vec{k}, \omega) k^{2}\left(i k_{i} \tilde{u}_{i}\right), \\
i k_{i} i k_{j} \stackrel{\tilde{\rho}}{P}_{i j}^{K}=n m \nu_{\ell}^{K}(\vec{k}, \omega) k^{2}\left(i k_{i} \tilde{u}_{i}\right)+\Gamma_{2}^{K}(\vec{k}, \omega) \frac{1}{T} k^{4} \tilde{T}, \\
\mathbb{Q}_{t}\left(i k_{j} \stackrel{\tilde{P}}{P}_{i j}^{K}\right)=n m \nu_{t}^{K}(\vec{k}, \omega) k^{2} \tilde{u}_{i_{t}} .
\end{gathered}
$$

Al expresar las ecuaciones de relajamiento de esta manera, podemos identificar directamente a los "coeficientes de transporte generalizados", que toman en cuenta la respuesta no-local y no instantánea del sistema. Estos coeficientes tienen una dependencia explícitia en el vector de onda y la frecuencia. El superíndice $\left({ }^{K}\right)$ nos indica que estas ecuaciones solamente incorporan el transporte cinético de los flujos. De hecho este conjunto de ecuaciones define sólo la contribución cinética de los coeficientes de transporte generalizados y es importante notar que las ecuaciones constitutivas nos dicen como es el comportamiento de los flujos cinéticos cuando se tiene inhomogeneidad en la temperatura, o bien fluctuaciones en la velocidad hidrodinámica.

La contribución completa al tensor de presiones requiere incorporar los flujos colisionales en las expresiones anteriores. En la aproximación a primer 
orden, la representación en 13-momentos para los flujos colisionales es:

$$
\begin{aligned}
\tilde{\stackrel{s}{P}}_{i j}^{I}(\vec{k}, \omega) & =\frac{2}{5} \chi b \rho \tilde{\tilde{P}}_{i j}^{K}(\vec{k}, \omega) \\
\tilde{q}_{i}^{I}(\vec{k}, \omega) & =\frac{3}{5} \chi b \rho \tilde{q}_{i}^{K}(\vec{k}, \omega)
\end{aligned}
$$

Estas ecuaciones representan un resultado muy importante de nuestro tratamiento. De hecho nos están diciendo que la trasferencia por colisiones es proporcional a la transferencia cinética. Dicho de otra forma, la transferencia por colisión no es independiente y por lo tanto basta con tomar a las partes cinéticas del tensor de presiones y del flujo de calor para obtener una descripción completa de los flujos totales. De manera que con ayuda de estas expresiones podemos escribir las ecuaciones (3.20)-(3.23) como:

$$
\begin{aligned}
\tilde{\stackrel{\rho}{P}}_{i j_{\text {total }}}(\vec{k}, \omega) & =\tilde{\stackrel{o}{P}}_{i j}^{K}(\vec{k}, \omega)+\tilde{\stackrel{o}{P}}_{i j}^{I}(\vec{k}, \omega) \\
& =\chi^{(2)} \stackrel{\tilde{s}}{P}_{i j}^{K}(\vec{k}, \omega), \\
\tilde{q}_{i_{\text {total }}}(\vec{k}, \omega) & =\tilde{q}_{i}^{K}(\vec{k}, \omega)+\tilde{q}_{i}^{I}(\vec{k}, \omega) \\
& =\chi^{(3)} \tilde{q}_{i}^{K}(\vec{k}, \omega) .
\end{aligned}
$$

Estas expresiones representan el flujo total de ímpetu y energía en gases de Enskog para la región de vectores de onda y frecuencia finitos.

Ahora nos interesa encontrar las ecuaciones constitutivas que corresponden a los flujos totales, mismos que se encuentran cuando se desacoplan los componentes longitudinal y transversal del tensor de presiones y del flujo de calor, para expresar las ecuaciones de relajamiento en la forma de ecuaciones constitutivas generalizadas que tengan la estructura de las ecuaciones (3.24)(3.26) éstas últimas se sustituyen en las ecuaciones (3.29)-(3.30) para obtener que,

$$
\begin{gathered}
i k_{i} \tilde{q}_{i_{\text {total }}}=\lambda(\vec{k}, \omega) k^{2} \tilde{T}-\Gamma(\vec{k}, \omega) k^{2}\left(i k_{i} \tilde{u}_{i}\right), \\
i k_{i} i k_{j} \tilde{\stackrel{\rho}{P}}_{i j_{\text {total }}}=n m \nu_{\ell}(\vec{k}, \omega) k^{2}\left(i k_{i} \tilde{u}_{i}\right)+\Gamma(\vec{k}, \omega) \frac{k^{4}}{T} \tilde{T} \\
\mathbb{Q}_{t} \cdot\left(i k_{j} \tilde{\stackrel{\rho}{P}}_{i j_{\text {total }}}\right)=n m \nu_{t}(\vec{k}, \omega) k^{2} \tilde{u}_{i_{t}} .
\end{gathered}
$$


Este conjunto de ecuaciones define a los coeficientes generalizados en el espacio de vectores de onda y frecuencia, cada coeficiente representa la respuesta del sistema ante las perturbaciones causadas en él.

Como ya hemos visto, insistimos en que es necesario considerar el flujo completo de ímpetu y energía, los cuales contienen una contribución por movimiento molecular, y otra por transferencia colisional. De hecho, esta consideración se debe hacer para cualquier sistema de alta densidad ya sea que se trate de gases densos o líquidos $[1,6]$. Esta situación la hemos hecho explícita en la construcción de las relaciones constitutivas generalizadas anteriores, donde definimos coeficientes de transporte generalizados en términos del tensor de presiones total y del flujo de calor total.

Para encontrar las expresiones explicitas de los coeficientes de transporte generalizados, se efectúan los pasos algebraicos señalados antes y obtenemos: la conductividad térmica generalizada

$$
\begin{aligned}
\lambda(\vec{k}, \omega)=\chi^{(3)} \Lambda^{K}(\vec{k}, \omega)= & \\
& =\lambda_{0} \frac{\chi^{(3)^{2}}}{\chi}\left\{1-i \omega \tau_{q}+\frac{8 k_{B} T}{15 m} \chi^{(3)^{2}} \frac{\tau_{p} \tau_{q} k^{2}}{\left(1-i \omega \tau_{p}\right)}\right\}^{-1},
\end{aligned}
$$

la parte longitudinal de la viscosidad cortante generalizada $\nu_{\ell}(\vec{k}, \omega)=\chi^{(2)} \nu_{\ell}^{K}(\vec{k}, \omega)=$

$$
=\frac{4}{3} \frac{\nu_{0}}{n m} \frac{\chi^{(2)^{2}}}{\chi}\left\{1-i \omega \tau_{p}+\frac{8 k_{B} T}{15 m} \chi^{(3)^{2}} \frac{\tau_{p} \tau_{q} k^{2}}{\left(1-i \omega \tau_{q}\right)}\right\}^{-1}
$$

la parte transversal de la viscosidad cortante generalizada $\nu_{t}(\vec{k}, \omega)=\chi^{(2)} \nu_{\ell}^{K}(\vec{k}, \omega)=$

$$
=\frac{\eta_{0}}{n ! m} \frac{\chi^{(2)^{2}}}{\chi}\left\{1-i \omega \tau_{p}+\frac{2 k_{B} T}{5 m} \chi^{(3)^{2}} \frac{\tau_{p} \tau_{q} k^{2}}{\left(1-i \omega \tau_{q}\right)}\right\}^{-1}
$$

y por último el coeficiente $\Gamma$ generalizado

$$
\begin{aligned}
\Gamma(\vec{k}, \omega) & =\chi^{(2)} \Gamma_{2}^{K}(\vec{k}, \omega)=\chi^{(3)} \Gamma_{1}^{K}(\vec{k}, \omega) \\
& =\frac{16 T}{45} \frac{\chi^{(2)} \chi^{(3)} \tau_{q}}{\left(1-i \omega \tau_{p}\right)} \Lambda^{K}(\vec{k}, \omega) .
\end{aligned}
$$


Este último coeficiente generalizado representa un efecto cruzado entre las partes longitudinales del tensor de presiones y el flujo de calor, el cual satisface una clase de simetría semejante a la que se encuentra en gases diluidos.

Estas expresiones de los coeficientes de transporte tienen algunos límites interesantes, por ejemplo, en el régimen diluido de bajas densidades, $n a^{3} \rightarrow 0$ las correlaciones espaciales $\chi, \chi^{(\nu)}, \ldots$ tienden a la unidad de tal forma que los coeficientes generalizados adquieren una estructura conocidla. Si regresamos a las ecuaciones completas (3.34)-(3.37) vemos que en el límite hidrodinámico usual de bajas frecuencias y longitudes de onda larga, $|\vec{k}| \rightarrow 0, \omega \rightarrow 0$, los coeficientes generalizados se reducen a:

$$
\begin{gathered}
\lim _{\substack{\mid \vec{k} \rightarrow 0 \\
\omega \rightarrow 0}} \lambda(\vec{k}, \omega)=\lambda_{0} \frac{\chi^{(3)^{2}}}{\chi}, \\
\lim _{\substack{|\vec{k}| \rightarrow 0 \\
\omega \rightarrow 0}} \nu_{\ell}(\vec{k}, \omega)=\frac{4 \eta_{0}}{3 n m} \frac{\chi^{(2)^{2}}}{\chi}, \\
\lim _{\substack{|\vec{k}| \rightarrow 0 \\
\omega \rightarrow 0}} \nu_{t}(\vec{k}, \omega)=\frac{\eta_{0}}{n m} \frac{\chi^{(2)^{2}}}{\chi}, \\
\lim _{\substack{|\vec{k}| \rightarrow 0 \\
\omega \rightarrow 0}} \Gamma(\vec{k}, \omega)=\frac{16 T}{45} \frac{\chi^{(2)} \chi^{(3)^{2}}}{\chi} \tau_{q} \lambda_{0} .
\end{gathered}
$$

Debemos notar que en el límite hidrodinámico recuperamos, como caso particular, los coeficientes de transporte usuales que se obtienen mediante el método de Chapman-Enskog [44]. Notemos también el coeficiente cruzado de termostricción tiene un límite diferente de cero, lo cual implica que los efectos de las inhomogeneidades en la temperatura afecta al tensor viscoso y a su vez, la rapidez de deformación medida por $i k_{i} u_{i}$ también tienen un efecto sobre el flujo de calor. Estos efectos son poco usuales debido a que cuando estamos interesados en medir un coeficiente de transporte generalmente se procura que éste sea el único efecto presente. Por ejemplo, cuando se estudia a la conductividad térmica se evita el flujo de fluido e inversamente, cuando se quiere ver el efecto del flujo se pone en condiciones de temperatura constante, evitando con ello las correcciones producidas por los términos que involucran efectos cruzados.

Finalmente, mencionaremos que la estructura de los coeficientes generalizados al ser muy parecida a los coeficientes de gases diluidos, nos permite 
hacer discusiones análogas con respecto a las modificaciones que tienen al variar los parámetros característicos. En particular, es necesario enfatizar la importancia de los tiempos de relajación, los cuales definen etapas diferentes de evolución y determinan el comportamiento con respecto a los vectores de onda $y$ frecuencias.

Los coeficientes generalizados muestran algunas diferencias importantes con respecto al caso diluido principalmente porque la densidad es muy importante en esta descripción, introduciendo algunos factores que definen las correlaciones espaciales. Ahora bien, estamos interesados en describir la dependencia de los coeficientes de transporte con la densidad, para ver el cambio cualitativo que tienen en la región de $(\vec{k}, \omega)$-finito. Esta dependencia se encuentra en las ecuaciones (3.34)-(3.37) a través de las funciones $\chi$. En la literatura no existe un acuerdo general en cuanto al intervalo de densidades para las cuales es válida la Ecuación de Enskog [30]. Para nosotros será de interés considerar el régimen diluido y moderadamente denso. Es costumbre en la literatura expresar el intervalo de densidades en términos de la llamada densidad reducida $\delta_{0}=\rho b$, donde se ha tomado como referencia la densidad correspondiente al caso del empaquetamiento máximo. En términos de esta densidad reducida el régimen diluido corresponde a $\delta_{0} \leq 0.1$ y un caso típico de gases moderadamente densos está localizado en $\delta_{0} \simeq 0.2$, de manera que éste será el intervalo que vamos a considerar.

Algunos rasgos generales del comportamiento de los coeficientes de transporte generalizados son:

Considerando su variación con respecto al vector de onda a frecuencia cero observamos de las figuras 3.1-3.6 que, en general, descienden suavemente con el aumento de $\kappa=k a$, en forma similar al caso del gas diluido $[38,39]$. Los coeficientes de transporte que recuperamos en el límite de $\kappa$-pequeña toman valores cada vez mayores con el aumento de la densidad; en mayor proporción aumenta la conductividad térmica, luego el coeficiente cruzado y mucho menos las viscosidades.

En estas curvas el coeficiente cruzado se encuentra entre la conductividad térmica por arriba y la viscosidad longitudinal por abajo. Esta cualidad se mantiene en toda la región de vectores de onda independientemente de la densidad. Las curvas nunca se cruzan manteniendo sus posiciones relativas desde el régimen hidrodinámico. La viscosidad transversal generalizada desciende más despacio que los otros coeficientes para vectores de onda pequeños, hasta 
Generalized Transport Coefficients



Figure 1: Coeficientes de transporte generalizados como funciones de $k a$ a diferentes densidades. La línea sólida representa la conductividad térmica y la línea con cuadros es el coeficiente cruzado. Las líneas a trazos y punteada representan las viscosidades transversal y longitudinal respectivamente a una densidad reducida de 0.125 . 
Generalized Transport Coefficients

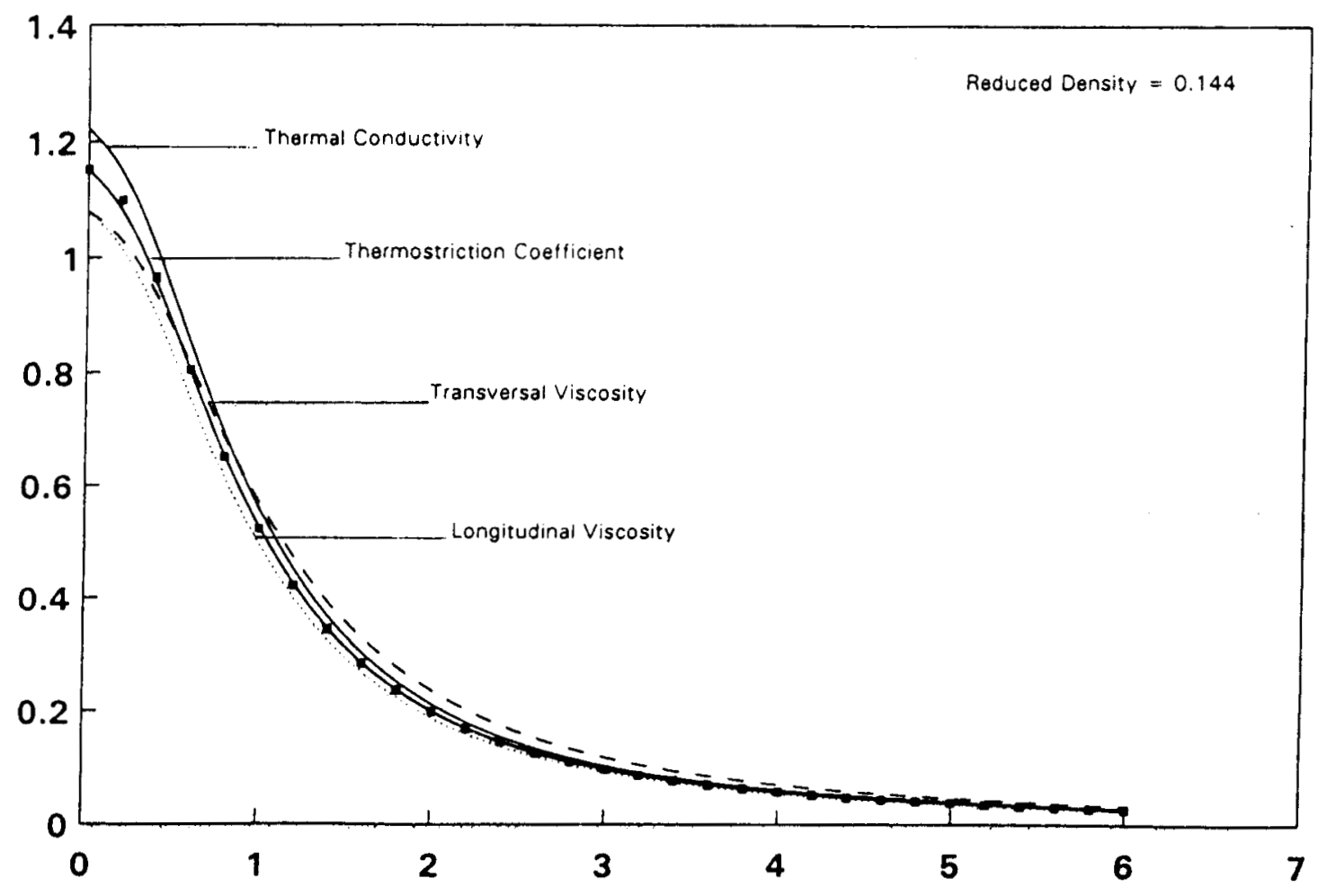

Figure 2: Coeficientes de transporte generalizados como funciones de $k a$ a la densidad reducidla de 0.144 . 
Generalized Transport Coefficients



Figure 3: Coeficientes de transporte generalizados como funciones de $k a$ a la densidad reducida de 0.200 .

que a un determinado valor de $\kappa$, intersecta primero a la curva del coeficiente cruzado y luego a la conductividad térmica de tal manera que en la región $\kappa \geq \kappa_{\text {cruce }}$ se mantiene ligeramente por encima de los demás coeficientes generalizados. Insistimos en que estas características generales se cumplen para todas las densidades que estamos considerando, incluyendo algunas del régimen diluido y otras a densidades moderadas, tales como $\delta_{0} \sim 0.075$ y $\delta_{0} \sim 0.144$ respectivamente. Otra característica importante de los coeficientes generalizados es que conforme aumenta la densidad las curvas se hacen más planas, de tal manera que para descender a un porcentaje determinado de su valor inicial se necesita cubrir un intervalo cada vez mayor de vectores 
de onda.

Comparando el comportamiento de los coeficientes generalizados con los resultados de dinámica molecular [32], observamos que para valores pequeños del vector de onda, digamos $i \leq 1.2$, la viscosidad transversal es ligeramente mayor a la correspondiente viscosidad de Dinámica Molecular, los resultados están en buen acuerdo con este intervalo que contiene al régimen hidrodinámico. Por otro lado para $\kappa \geq 1.2$ los puntos Dinámica Molecular sugieren un punto de cruce un poco después de $\kappa \simeq 1$, quedando sus resultados, para vectores de onda mayores, un poco por encima de la curva correspondiente. Una corrección a esta tendencia de permanecer las curvas a una cierta altura para valores del vector de onda relativamente grandes la hemos encontrado dentro de nuestro modelo cinético incorporando en la descripción a los flujos colisionales de orden superior. Aún así, creemos que la discrepancia en los resultados se debe a que en dinámica molecular no se pueden separar adecuadamente los efectos cruzados y éstos pueden quedar incluidos como contribuciones escondidas en los coeficientes de transporte usuales. El hecho de que las curvas sean planas, para $\kappa$-pequeñas con un hombro pronunciado en ésta región sugiere la transición suave del régimen hidrodinámico al régimen cinético.

Ahora consideremos el comportamiento de la parte real de los coeficientes generalizados con respecto a la frecuencia, a vector de onda cero, y a una densidad moderadamente elevada, $\delta_{0}=0.144$. En este caso los coeficientes tienen valores considerablemente más grandes que los del gas diluido en el régimen hidrodinámico y sobre todo el intervalo de frecuencias considerado.

Es importante señalar que el descenso porcentual de los máximos se lleva a cabo a frecuencias solo ligeramente más grandes que las correspondientes al gas diluido. Esto parece indicar que el descenso de los coeficientes generalizados respecto a la frecuencia en un gas denso no es muy diferente que en gases diluidos [38,39].

Específicamente el corrimiento de la frecuencia, respecto del gas diluido, para la cual los coeficientes generalizados se han reducido a una décima parte de su valor inicial es de un $16 \%, 19 \%$ y $14 \%$ hacia valores mayores de la frecuencia para la conductividad térmica, el coeficiente cruzado, y la viscosidad respectivamente. Las viscosidades longitudinal y transversal, así como la conductividad térmica tienen un comportamiento de descenso suave al aumentar la frecuencia. El coeficiente cruzado desciende muy rápido pasando a tomar valores negativos en $\omega \tau_{p} \sim 1$ hasta alcanzar un mínimo en $\omega \tau_{p} \sim 1.5$, 
Comparison with Molecular Dynamics Data.

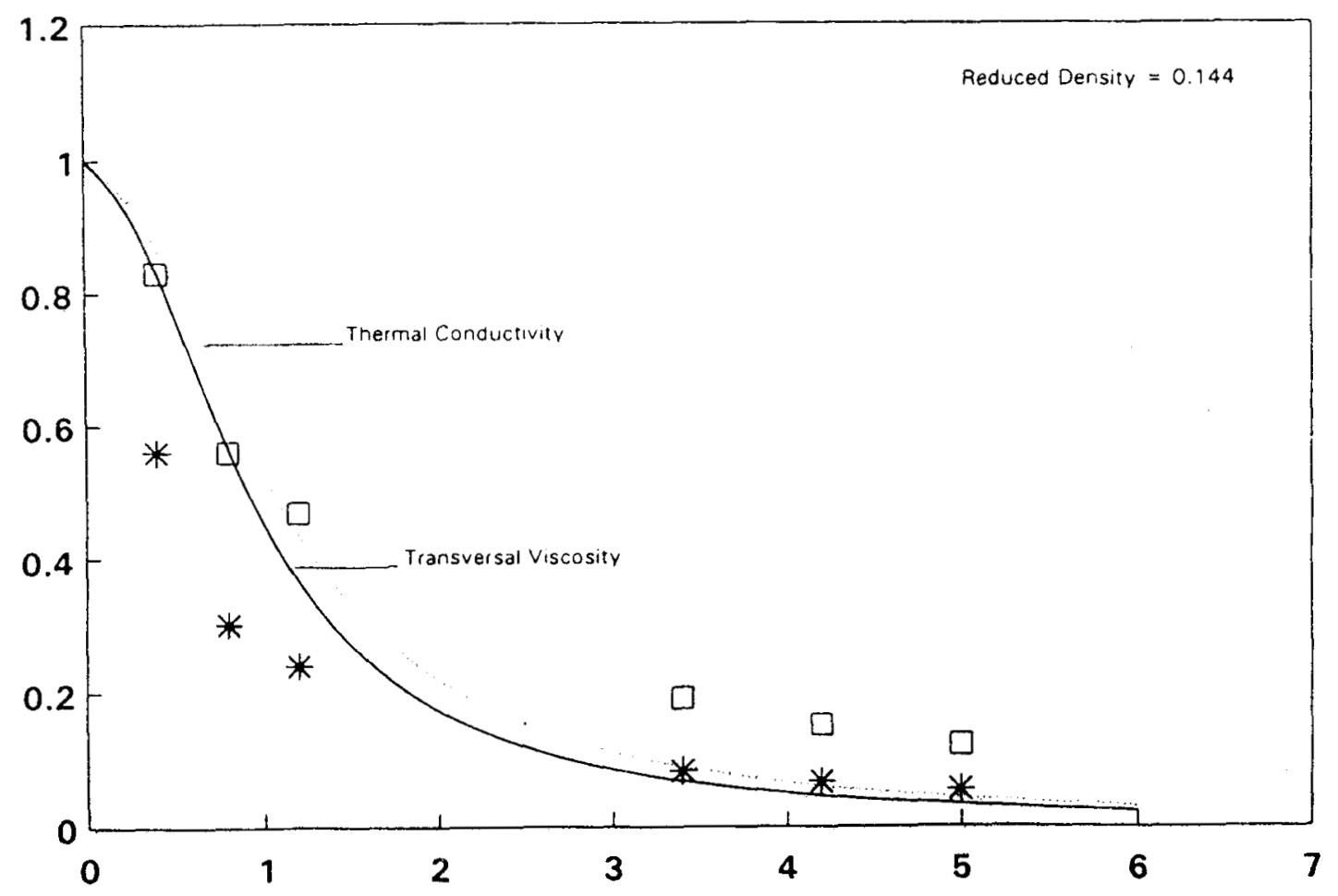

Figure 4: Comparación con los cálculos de dinámica molecular. La línea punteada corresponde a la conductividad térmica normalizada, mientras que las estrellas los cálculos de dinámica molecular. La línea sólida es la viscosidad longitudinal mientras que los cuadros son los resultados de dinámica molecular. Los coeficientes generalizados son función del vector de onda. 
Real part of generalized coefficients.

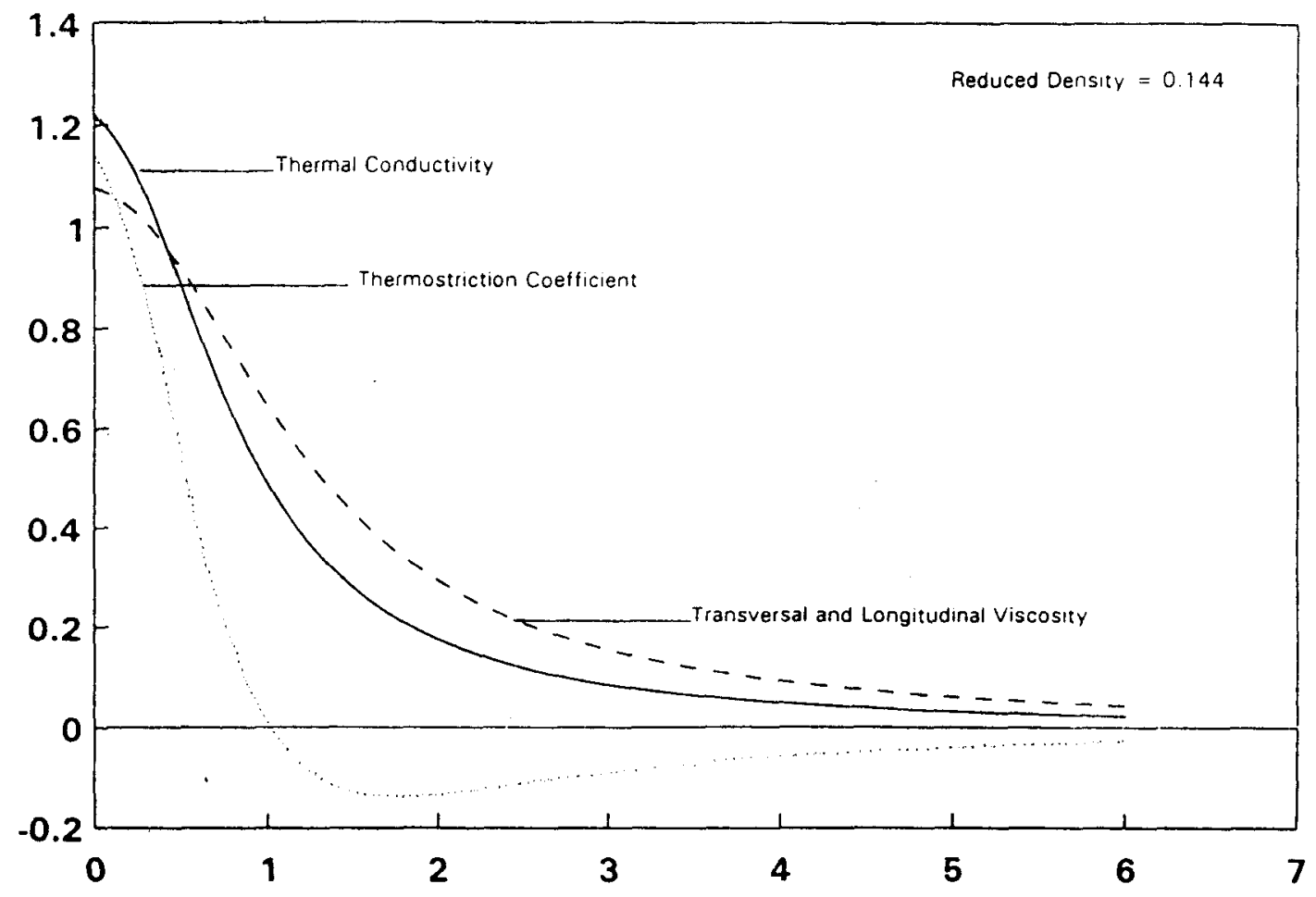

Figure 5: Parte real de los coeficientes de transporte generalizados como funciones de la frecuencia adimensional $\omega \tau_{p}$. La línea sólida representa la conductividad térmica y la punteada al coeficiente cruzado. La línea a trazos corresponde a las viscosidades longitudinal y transversal. 
Imaginary part of transport coefficients

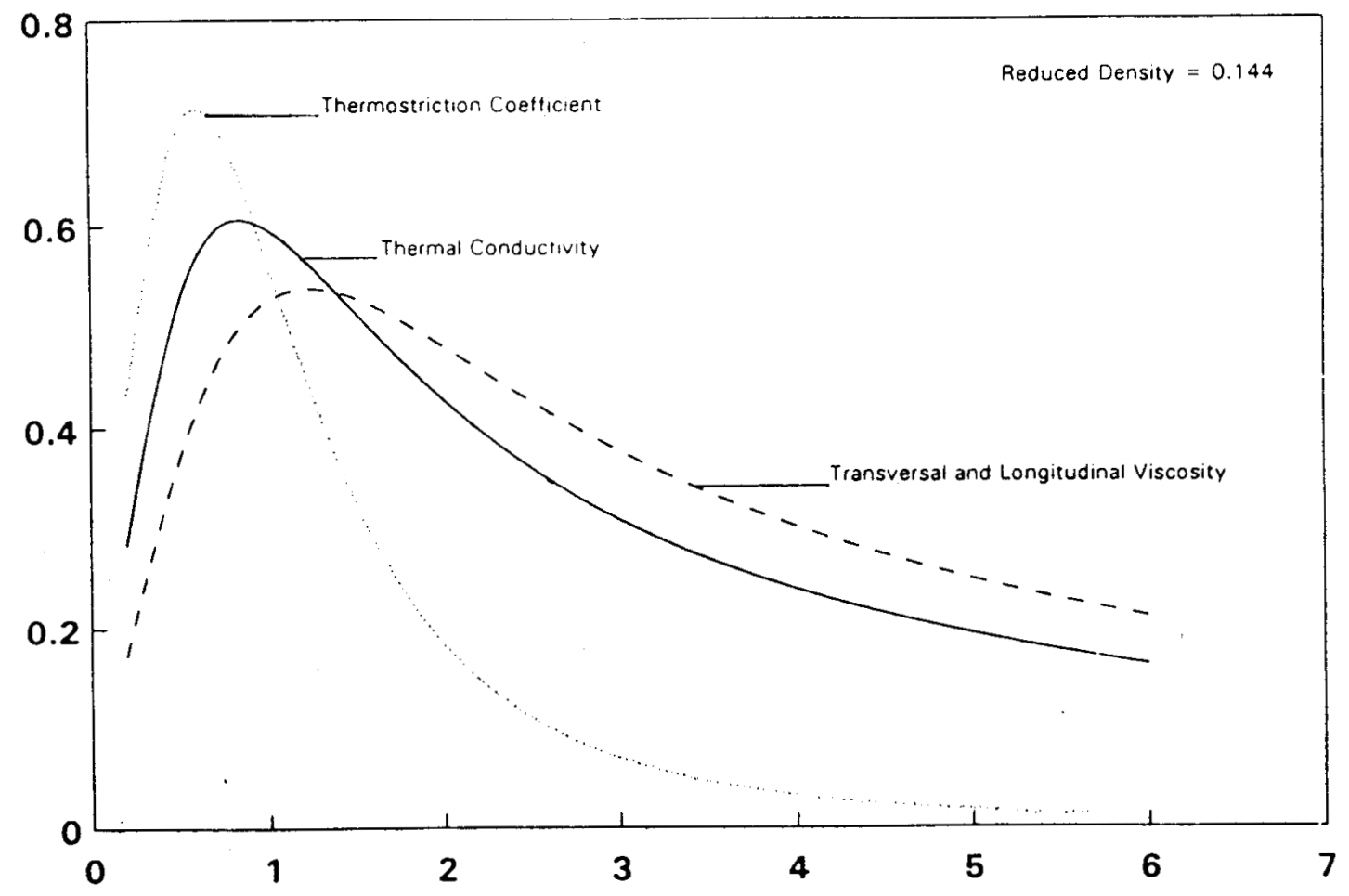

Figure 6: Parte imaginaria de los coeficientes generalizados como funciones de $\omega \tau_{p}$. La línea sólida es la conductividad térmica y la línea punteada al coeficiente cruzado. La línea a trazos representa tanto a la viscosidad longitudinal como transversal. 
para después ascender asintóticamente al eje de las frecuencias.

El comportamiento general de la parte imaginaria de los coeficientes generalizados con respecto a la frecuencia mantiene los rasgos generales de las curvas del gas diluido. Al aumentar la densidad de los coeficientes generalizados toman valores ligeramente más grandes con respecto al gas diluido sin cambiar esencialmente la forma de las curvas. Esto sugiere que en la región de altas frecuencias, los procesos de relajación son los mismos para ambos sistemas, en el sentido que los procesos moleculares de transporte no cambian, pero en densidades elevadas las colisiones son más frecuentes. También en este caso ocurre un corrimiento en el descenso porcentual de los coeficientes generalizados hacia valores un poco mayores en la frecuencia con respecto al gas diluido. La frecuencia en la cual el máximo del coeficiente cruzado se reduce a una décima parte es $\omega \tau_{p} \sim 2.9$, mientras que en el gas diluido sucede a $\omega \tau_{p} \sim 2.6$. Para la viscosidad cortante y longitudinal el corrimiento en la frecuencia necesario para que el máximo bajo a un $50 \%$ es $\omega \tau_{p} \sim 4.67$ que para el diluido $\omega \tau_{p} \sim 4.0$. Finalmente para la conductividad térmica $\omega \tau_{p} \sim 5.5$ es el valor al cual su máximo se reduce en un $30 \%$ en comparación con el gas diluido, esto sucede a $\omega \tau_{p} \sim 4.7$. Como podemos ver las frecuencias se recorren en un $12 \%, 17 \%$ y $18 \%$ solamente para el coeficiente cruzado, la viscosidad y la conductividad térmica respectivamente.

Todos los coeficentes están normalizados con respecto a sus valores en el régimen hidrodinámico del gas diluido. Cada figura corresponde a un valor diferente en la densidad reducida, todas ellas dentro del intervalo de validez de la ecuación de Enskog.

El análisis anterior del comportamiento de los coeficientes de transporte generalizados nos permite conocer las diferencias porcentuales que surgen en los procesos de transporte con el aumento de la densidad. En efecto, conociendo el comportamiento de las curvas al variar el vector de onda o la frecuencia, es posible hacer una comparación con los coeficientes generalizados en gases diluidos, de tal manera que las diferencias porcentuales correspondientes son las contribuciones por transferencia colisional. 


\section{Capítulo 4}

\section{Coeficientes de Transporte Generalizados}

\section{Segundo Orden en los Flujos Colisionales}

En el capítulo anterior hemos calculado un conjunto de relaciones constitutivas generalizadas donde los coeficientes de transporte usuales ahora son funciones del vector de onda y la frecuencia. Estas relaciones contienen a las ecuaciones constitutivas usuales del régimen hidrodinámico como caso particular en el límite $(k \rightarrow 0, \omega \rightarrow 0)$. En este sentido, resalta el hecho de que no hemos obtenido un coeficiente del tipo viscosidad volumétrica generalizada, el cual aparece en una descripción usual de la hidrodinámica. Este coeficiente es del orden de la densidad al cuadrado, y para obtenerlo es necesario extender el modelo cinético completo hasta segundo orden en los flujos colisionales $[44,45,64]$. Este procedimiento incorpora correcciones pequeñas a los coeficientes generalizados de la sección anterior, lo cual se manifiesta con mayor importancia para valores grandes del vector de onda y la frecuencia; sin embargo, en la parte diagonal del tensor de presiones nos conduce a un coeficiente de viscosidad volumétrica generalizado el cual tiene el comportamiento bien conocido en el régimen hidrodinámico.

\subsection{Ecuaciones de movimiento en el espacio $(\vec{k}, \omega)$}

Las ecuaciones de movimiento linealizadas para las variables no conservadas, tal como la parte cinética del tensor de presiones $\stackrel{\circ}{P}_{i j}^{K}$ y el flujo de calor $q_{i}^{K}$ en la aproximación a segundo orden en los flujos colisionales, tienen una solución en el estado de equilibrio termodinámico, donde el sistema se encuentra en reposo y no hay procesos de relajamiento macroscópicos. Las ecuaciones de evolución para perturbaciones pequeñas de las variables relevantes alrededor del estado de equilibrio son;

$$
\frac{\partial \rho^{\prime}}{\partial t}+\rho \frac{\partial u_{i}^{\prime}}{\partial x_{i}}=0
$$




$$
\begin{aligned}
& \rho \frac{\partial u_{i}^{\prime}}{\partial t}+\frac{\chi^{(\rho)}}{\alpha} \frac{\partial \rho^{\prime}}{\partial x_{i}}-\rho \tilde{\omega}_{0}\left\{\frac{1}{2} \frac{\partial^{2} u_{i}^{\prime}}{\partial x_{k} \partial x_{k}}+\frac{\partial^{2} u_{k}^{\prime}}{\partial x_{k} \partial x_{i}}\right\}+ \\
& +\frac{\rho}{\alpha T} \chi^{(5)} \frac{\partial T^{\prime}}{\partial x_{i}}+\chi^{(2)} \frac{\partial \dot{P}_{i j}^{K^{\prime \prime}}}{\partial x_{j}}-\frac{\alpha \tilde{\omega}_{0}}{10}\left\{\frac{1}{2} \frac{\partial^{2} q_{i}^{K^{\prime \prime}}}{\partial x_{k} \partial x_{k}}+\frac{\partial^{2} q_{k}^{K^{\prime \prime}}}{\partial x_{k} \partial x_{i}}\right\}=0,
\end{aligned}
$$

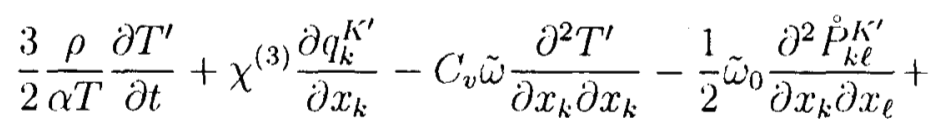

$$
\begin{aligned}
& +\frac{\rho}{\alpha} \chi^{(5)} \frac{\partial u_{k}^{\prime}}{\partial x_{k}}=0 \\
& \frac{\partial \stackrel{\circ}{P}_{i j}^{K^{\prime}}}{\partial t}-\frac{4}{7} \tilde{\omega}_{0}\left(\frac{\partial^{2} \stackrel{\circ}{P}_{k i}^{K^{\prime}}}{\partial x_{k} \partial x_{j}}\right)^{0}-\frac{1}{7} \tilde{\omega}_{0} \frac{\partial^{2} \stackrel{\circ}{P}_{i j}^{K^{\prime}}}{\partial x_{k} \partial x_{k}}+2 \chi^{(2)} \frac{\rho}{\alpha}\left(\frac{\partial u_{i}^{\prime}}{\partial x_{j}}\right)^{0}+ \\
& +\frac{4}{5} \chi^{(3)}\left(\frac{\partial q_{i}^{K^{\prime}}}{\partial x_{j}}\right)^{0}-\frac{4}{5} c_{i} \tilde{\omega}\left(\frac{\partial^{2} T^{\prime}}{\partial x_{j} \partial x_{i}}\right)^{0}=-\frac{1}{\tau_{p}} \stackrel{P}{P}_{i j}^{K^{\prime \prime}}
\end{aligned}
$$

y

$$
\begin{aligned}
& \frac{\partial q_{i}^{K^{\prime}}}{\partial t}-\frac{2}{3} \tilde{\omega}_{0} \frac{\partial^{2} q_{k}^{K^{\prime}}}{\partial x_{k} \partial x_{i}}-\frac{1}{3} \tilde{\omega}_{0} \frac{\partial q_{i j}^{K^{\prime \prime}}}{\partial x_{k} \partial x_{k}}+\frac{5 \rho}{2 \alpha^{2} T} \chi^{(3)} \frac{\partial T^{\prime}}{\partial x_{i}}+ \\
& +\frac{\chi^{(3)}}{\alpha} \frac{\partial \dot{\rho}_{i j}^{K^{\prime \prime}}}{\partial x_{j}}-\frac{1}{8} \frac{\rho}{\alpha} \tilde{\omega}_{0} \frac{\partial^{2} u_{i}^{\prime}}{\partial x_{k} \partial x_{k}}-\frac{1}{4} \frac{\rho}{\alpha} \tilde{\omega}_{0} \frac{\partial^{2} u_{k}}{\partial x_{k} \partial x_{i}}=-\frac{1}{\tau_{q}} q_{i}^{K^{\prime}} .
\end{aligned}
$$

Estas ecuaciones se obtienen a partir de (2.71)-(2.75); las primeras corresponden a las variables conservadas, mientras que las últimas son las ecuaciones de relajamiento para las fluctuaciones del tensor de presiones y del flujo de calor. Los coeficientes que aparecen aquí, están definidos en las ecuaciones (2.76)(2.81), mientras que las variables sin prima están evaluadas en el estado de equilibrio termodinámico por lo culal son cantidades constantes. Como podemos observar, estas ecuaciones contienen términos a segundo orden en los gradientes de los flujos colisionales y todos ellos se caracterizan por la presencia de la "viscosidad volumétrica" del régimen hidrodinámico [44,45]. Las ecuaciones de evolución de las variables no-conservadas, es decir, las ecuaciones de relajamiento (4.4) y (4.5), dan origen a los coeficientes de transporte generalizados $[38,39]$. 
En forma similar al cálculo a primer orden en los flujos colisionales, podemos expresar;

$$
\begin{aligned}
& \frac{\partial{\stackrel{\stackrel{P}{P}}{i_{j}}}^{K^{\prime}}}{\partial t}= \\
& -\frac{1}{\tau_{p}}\left[\stackrel{\circ}{P}_{i j}^{K^{\prime \prime}}-\frac{4}{7} \tilde{\omega}_{0} \tau_{p}\left(\frac{\partial^{2} \stackrel{\circ}{K_{k i}^{K^{\prime}}}}{\partial x_{k} \partial x_{j}}\right)^{0}-\frac{1}{7} \tilde{\omega}_{0} \tau_{p} \frac{\partial^{2} \stackrel{\circ}{P}_{i j}^{K^{\prime \prime}}}{\partial x_{k} \partial x_{k}}+2 \eta_{0} \frac{\chi^{(2)}}{\chi}\left(\frac{\partial u_{i}^{\prime}}{\partial x_{j}}\right)^{0}\right]- \\
& -\frac{4}{5}\left[\chi^{(3)}\left(\frac{\partial q_{i}^{K^{\prime}}}{\partial x_{j}}\right)^{0}-c_{v} \tilde{\omega}\left(\frac{\partial^{2} T^{\prime}}{\partial x_{j} \partial x_{i}}\right)^{0}\right] \\
& \frac{\partial q_{i}^{K^{\prime}}}{\partial t}= \\
& -\frac{1}{\tau_{q}}\left[q_{i}^{K^{\prime \prime}}-\frac{2}{3} \tilde{\omega}_{0} \tau_{q} \frac{\partial^{2} q_{k}^{K^{\prime \prime}}}{\partial x_{k} \partial x_{i}}-\frac{1}{3} \tilde{\omega}_{0} \tau_{q} \frac{\partial^{2} q_{i}^{K^{\prime \prime}}}{\partial x_{k} \partial x_{k}}+\lambda_{0} \frac{\chi^{(3)}}{\chi} \frac{\partial T^{\prime}}{\partial x_{i}}\right]- \\
& -\left[\frac{\chi^{(3)}}{\alpha} \frac{\partial \dot{P}_{i j}^{K^{\prime \prime}}}{\partial x_{j}}-\frac{1}{8} \frac{\rho}{\alpha} \tilde{\omega}_{0} \frac{\partial^{2} u_{i}^{\prime}}{\partial x_{k} \partial x_{k}}-\frac{1}{4} \frac{\rho}{\alpha} \tilde{\omega}_{0} \frac{\partial^{2} u_{k}^{\prime}}{\partial x_{k} \partial x_{i}}\right] .
\end{aligned}
$$

Estas ecuaciones son una extensión de las ecuaciones (3.6) y (3.7) de la aproximación a primer orden, en la cual $\tilde{\omega}_{0}=0$.

Para obtener coeficientes de transporte generalizados, podemos proceder en forma similar al caso anterior. En efecto, vamos a tomar la transformada de Fourier de las ecuaciones (4.6) y (4.7) y sus proyecciones, longitudinales y transversales con respecto a la dirección del vector de onda. Con ayuda de los operadores de proyección definidos anteriormente obtenemos:

$$
-i \omega \tilde{\rho}+\rho\left(i k_{i} \tilde{u}_{i}\right)=0,
$$

para la componente longitudinal de la velocidlad, y

$$
\begin{aligned}
& -i \omega \rho\left(i k_{i} \tilde{u}_{i}\right)+\frac{\chi^{(\rho)}}{\alpha}(i k)^{2} \tilde{\rho}-\frac{3}{2} \rho \tilde{\omega}_{0}(i k)^{2}\left(i k_{i} \tilde{u}_{i}\right)+ \\
& +\frac{\rho}{\alpha T} \chi^{(5)}(i k)^{2} \tilde{T}+\chi^{(2)} i k_{i} i k_{j} \stackrel{\tilde{P}}{P}_{i j}^{K}-\frac{3}{20} \alpha \tilde{\omega}_{0}(i k)^{2}\left(i k_{i} \tilde{q}_{i}^{K}\right)=0,
\end{aligned}
$$


para las fluctuaciones en la temperatura,

$$
\begin{aligned}
& -i \omega \frac{3}{2} \frac{\rho}{\alpha T} \tilde{T}+\chi^{(3)}\left(i k_{i} \tilde{q}_{i}^{K}\right)-c_{i} \tilde{\omega}(i k)^{2} \tilde{T}-\frac{1}{2} \tilde{\omega}_{0} i k_{i} i k_{j} \tilde{s}_{i j}^{K} \\
& +\frac{\rho}{\alpha} \chi^{(5)}\left(i k_{i} \tilde{u}_{i}\right)=0,
\end{aligned}
$$

finalmente la componente transversal de la velocidad es,

$$
-i \omega \rho \tilde{u}_{i_{t}}-\frac{1}{2} \rho \tilde{\omega}_{0}(i k)^{2} \tilde{u}_{i_{t}}+\chi^{(2)} \mathbb{Q}_{t} \cdot\left(i k_{j} \tilde{P}_{i j}^{K}\right)-\frac{\alpha \tilde{\omega}_{0}}{20}(i k)^{2} \tilde{q}_{i_{t}}^{K}=0 .
$$

Considerando las ecuaciones (4.6) y (4.7) como las formas iniciales de las ecuaciones de relajamiento, separamos sus componentes longitudinales y transversales con respecto a la dirección del vector de onda. Con ayuda de los operadores de proyección obtenemos que para las componentes longitudinales de las ecuaciones de relajación en el espacio de Fourier,

$$
\begin{aligned}
& \left(1-i \omega \tau_{p}-\frac{1}{2} \tilde{\omega}_{0} \tau_{p}(i k)^{2}\right) i k_{i} i k_{j} \tilde{\stackrel{P}{P}}_{i j}^{K}=\frac{4}{3} \eta_{0} \frac{\chi^{(2)}}{\chi} k^{2}\left(i k_{i} \tilde{u}_{i}\right) \\
& +\frac{8}{15} \tau_{p}\left\{\chi^{(3)} k^{2}\left(i k_{i} \tilde{q}_{i}^{K}\right)+c_{v} \tilde{\omega} k^{4} \tilde{T}\right\}
\end{aligned}
$$

y

$$
\begin{aligned}
& \left(1-i \omega \tau_{q}-\tilde{\omega}_{0} \tau_{q}(i k)^{2}\right) i k_{i} \tilde{q}_{i}^{K}=\lambda_{0} \frac{\chi^{(3)}}{\chi} k^{2} \tilde{T}- \\
& -\tau_{q}\left\{\frac{\chi^{(3)}}{\alpha} i k_{i} i k_{j} \tilde{\stackrel{P}{P}}_{i j}^{K}+\frac{3}{8} \frac{\rho}{\alpha} \tilde{\omega} k^{2}\left(i k_{i} \tilde{u}_{i}\right)\right\} .
\end{aligned}
$$

En cuanto a las componentes transversales de las ecuaciones de relajación,

$$
\begin{aligned}
& \left(1-i \omega \tau_{p}-\frac{3}{7} \tilde{\omega}_{0} \tau_{p}(i k)^{2}\right) \mathbb{Q}_{t} \cdot\left(i k_{j} \tilde{\stackrel{s}{P}}_{i j}^{K}\right)=\eta_{0} \frac{\chi^{(2)}}{\chi} k^{2} \tilde{u}_{i_{t}} \\
& +\frac{2}{5} \tau_{p} \chi^{(3)} k^{2} \tilde{q}_{i_{t}}^{K} \\
& \left(1-i \omega \tau_{q}-\frac{1}{3} \tilde{\omega}_{0} \tau_{q}(i k)^{2}\right) \tilde{q}_{i_{t}}^{K}=-\frac{1}{8} \frac{\rho}{\alpha} \tau_{q} \tilde{\omega}_{0} k^{2} \tilde{u}_{i_{t}} \\
& -\tau_{q} \frac{\chi^{(3)}}{\alpha} \mathbb{Q}_{t} \cdot\left(i k_{j} \tilde{\stackrel{\rho}{P}}_{i j}^{K}\right) .
\end{aligned}
$$


Todas estas ecuaciones se reducen a las ecuaciones (3.16)-(3.2:3) cuando despreciamos los términos de segundo orden en los flujos colisionales, los cuales están caracterizados por la presencia de la viscosidad volumétric:a usual. Las variables de relajación contienen contribuciones importantes que provienen de sus inhomogeneidades, así como los gradientes de segundo orden de las variables conservadas. Una de las principales consecuencias de este hecho es la obtención de ecuaciones constitutivas para los flujos de ímpetu y energía los cuales están dados a segundo orden en la densidad.

En las ecuaciones (4.8)-(4.15) los coeficientes son constantes y corresponden a sus valores de equilibrio, las variables con tilde son las transformadas de Fourier de las cantidades correspondientes en el espacio real, por lo tanto, son funciones del vector de onda y la frecuencia. Las ecuaciones de relajamiento están escritas en tal forma que sus componentes longitudinales y transversales quedan separadas y son extensiones, a segundo orden en la densidad de las ecuaciones (2.16)-(2.23). Igual que en el caso anterior estas ecuaciones son el punto de partida para encontrar coeficientes de transporte generalizados.

\subsection{Ecuaciones constitutivas generalizadas}

Como ya hemos visto, las ecuaciones de relajamiento se pueden expresar en la forma de relaciones constitutivas generalizadas. Para esto desacoplamos las ecuaciones algebraicas (4.12)-(4.15) en forma independiente, expresando el tensor de presiones y el flujo de calor (parte cinética) en términos de los gradientes de las variables conservadlas elegidas. Este procedimiento es más complicado que el de primer orden, sin embargo, el resultado es formalmente el mismo:

$$
\begin{gathered}
i k_{i} \tilde{q}_{i}^{K}=\Lambda^{K}(\vec{k}, \omega) k^{2} \tilde{T}-\Gamma_{1}^{K}(\vec{k}, \omega) k^{2}\left(i k_{i} \tilde{u}_{i}\right), \\
i k_{i} i k_{j} \stackrel{\circ}{P}_{i j}^{K}=n m \nu_{\ell}^{K}(\vec{k}, \omega) k^{2}\left(i k_{i} \tilde{u}_{i}\right)+\Gamma_{2}^{K}(\vec{k}, \omega) \frac{1}{T} k^{4} \tilde{T}, \\
\mathbb{Q}_{t} \cdot\left(i k_{j} \stackrel{\tilde{D}}{P}_{i j}^{K}\right)=n m \nu_{t}^{K}(\vec{k}, \omega) k^{2} \tilde{u}_{i_{t}} .
\end{gathered}
$$

Al expresar las ecuaciones de relajamiento de esta manera podemos identificar directamente a los coeficientes de transporte generalizados. El superíndice $\left({ }^{K}\right)$ es estos coeficientes nos indica que solamente estamos tomando en cuenta la contribución cinética de los flujos. 
Por simplicidad vamos a introducir las siguientes definiciones:

$$
\begin{gathered}
\Lambda^{K}(\vec{k}, \omega)=\Lambda_{\alpha}^{K}(\vec{k}, \omega)+\Lambda_{\beta}^{K}(\vec{k}, \omega), \\
\Gamma_{1}^{K}(\vec{k}, \omega)=\Gamma_{1, \alpha}^{K}(\vec{k}, \omega)+\Gamma_{1, \beta}^{K}(\vec{k}, \omega), \\
n m \nu_{\ell}^{K}(\vec{k}, \omega)=n m \nu_{\ell, \alpha}^{K}(\vec{k}, \omega)+n m \nu_{\ell, \beta}^{K}(\vec{k}, \omega), \\
\Gamma_{2}^{K}(\vec{k}, \omega)=\Gamma_{2, \alpha}^{K}(\vec{k}, \omega)+\Gamma_{2, \beta}^{K}(\vec{k}, \omega), \\
n m \nu_{t}^{K}(\vec{k}, \omega)=n m \nu_{t, \alpha}^{K}(\vec{k}, \omega)+n m \nu_{t, \beta}^{K}(\vec{k}, \omega) .
\end{gathered}
$$

En general, las componentes $(\alpha)$ son generalizaciones de las expresiones que se obtienen a primer orden, las cuales podemos recuperar en el límite apropiado. Las componentes $(\beta)$ son correcciones pequeñas al término dominante que es de primer orden en la densidad, ésto para vectores de onda y frecuencias cercanas al régimen hidrodinámico, y todas ellas están caracterizadas por la presencia de la viscosidad volumétrica usual. Lo anterior nos indica que los coeficientes de transporte tienen una influencia mayor en el régimen hidrodinámico y que prácticamente su comportamiento en la región finita de $(\vec{k}, \omega)$ está regido por flujos colisionales a primer orden.

Para expresar los coeficientes de transporte generalizados, en forma compacta, introducimos la definición,

$$
\Lambda_{\alpha, a d}^{K}(\vec{k}, \omega)=\left\{\left(1-i \omega \tau_{q}+\tilde{\omega}_{0} \tau_{q} k^{2}\right)+\frac{8 k_{B} T}{15 m} \chi^{(3)} \frac{\tau_{p} \tau_{q} k^{2}}{\left(1-i \omega \tau_{p}+\frac{1}{2} \tilde{\omega}_{0} \tau_{p} k^{2}\right)}\right\}^{-1}
$$

la cual es una función de $(\vec{k}, \omega)$ que aparece al resolver el conjunto de ecuaciones de relajación, ecs.(4.12)-(4.15), de donde;

$$
\begin{gathered}
\Lambda_{\alpha}^{K}(\vec{k}, \omega)=\lambda_{0} \frac{\chi^{(3)}}{\chi} \cdot \Lambda_{\alpha, a d}^{K}(\vec{k}, \omega), \\
\Lambda_{\beta}^{K}(\vec{k}, \omega)=\left(-\frac{8 k_{B} T}{15 m}\right) \frac{\chi^{(3)} C_{v} \tilde{\omega} \tau_{p} \tau_{q} k^{2}}{\left(1-i \omega \tau_{p}+\frac{1}{2} \tilde{\omega}_{0} \tau_{p} k^{2}\right)} \Lambda_{\alpha, a d}^{K}(\vec{k}, \omega), \\
\Gamma_{1, \alpha}^{K}(\vec{k}, \omega)=\left(\frac{4 k_{B} T}{3 m}\right) \frac{\chi^{(2)} \chi^{(3)} \eta_{0} \tau_{q}}{\chi\left(1-i \omega \tau_{p}+\frac{1}{2} \tilde{\omega}_{0} \tau_{p} k^{2}\right)} \Lambda_{\alpha, a d}^{K}(\vec{k}, \omega),
\end{gathered}
$$




$$
\Gamma_{1, \beta}^{K}(\vec{k}, \omega)=\left(-\frac{3 k_{B} T}{8 m}\right) \rho \tilde{\omega}_{0} \tau_{q} \Lambda_{\alpha, a d}^{K}(\vec{k}, \omega)
$$

También podemos definir

$$
\epsilon_{\alpha, a d}^{K}(\vec{k}, \omega)=\left\{\left(1-i \omega \tau_{p}+\frac{1}{2} \tilde{\omega}_{0} \tau_{p} k^{2}\right)+\frac{8 k_{B} T}{15 m} \chi^{(3)^{2}} \frac{\tau_{p} \tau_{q} k^{2}}{\left(1-i \omega \tau_{q}+\tilde{\omega}_{0} \tau_{q} k^{2}\right)}\right\}^{-1}
$$

$\mathrm{y}$ entonces

$$
\begin{gathered}
n m \nu_{\ell_{\alpha}}^{K}(\vec{k}, \omega)=\frac{4}{3} \eta_{0} \frac{\chi^{(2)}}{\chi} \cdot \epsilon_{\alpha, a d}^{K}(\vec{k}, \omega), \\
n m \nu_{\ell_{\beta}}^{K}(\vec{k}, \omega)=\left(\frac{k_{B} T}{5 m}\right) \frac{\chi^{(3)} \rho \tilde{\omega}_{0} \tau_{p} \tau_{q} k^{2}}{\left(1-i \omega \tau_{q}+\tilde{\omega}_{0} \tau_{q} k^{2}\right)} \cdot \epsilon_{\boldsymbol{\alpha}, a d}^{K}(\vec{k}, \omega), \\
\Gamma_{2, \alpha x}^{K}(\vec{k}, \omega)=\left(\frac{8 T}{15}\right) \frac{\chi^{(3)^{2}} \lambda_{0} \tau_{p}}{\chi\left(1-i \omega \tau_{q}+\tilde{\omega}_{0} \tau_{q} k^{2}\right)} \cdot \epsilon_{\alpha, a d}^{K}(\vec{k}, \omega), \\
\Gamma_{2, \beta}^{K}(\vec{k}, \omega)=\left(\frac{8 T}{15}\right) C_{v} \tilde{\omega} \tau_{p} \cdot \epsilon_{\alpha, a d}^{K}(\vec{k}, \omega) .
\end{gathered}
$$

Haciendo

$$
\epsilon_{t \alpha, \text { ad }}^{K}(\vec{k}, \omega)=\left\{\left(1-i \omega \tau_{p}+\frac{3}{7} \tilde{\omega}_{0} \tau_{p} k^{2}\right)+\frac{2 k_{B} T}{5 m} \chi^{(3)^{2}} \frac{\tau_{p} \tau_{q} k^{2}}{\left(1-i \omega \tau_{q}+\frac{1}{3} \tilde{\omega}_{0} \tau_{q} k^{2}\right)}\right\}^{-1}
$$

se obtiene,

y

$$
n m \nu_{t, \alpha}^{K}(\vec{k}, \omega)=\eta_{0} \frac{\chi^{(2)}}{\chi} \cdot \epsilon_{t \alpha, a d}^{K}(\vec{k}, \omega)
$$

$$
n m \nu_{t, \beta}^{K}(\vec{k}, \omega)=\left(-\frac{k_{B} T}{20 m}\right) \frac{\chi^{(3)} \rho \tilde{\omega}_{0} \tau_{p} \tau_{q} k^{2}}{\left(1-i \omega \tau_{q}+\frac{1}{3} \tilde{\omega} \tau_{q} k^{2}\right)} \epsilon_{t \alpha, a d}^{K}(\vec{k}, \omega)
$$

Las relaciones constitutivas completias, involucran las contribuciones cinéticas y colisionales de los flujos. En el modelo cinético que desarrollamos en el capítulo 2 y que nos condujo a las ecuaciones de movimiento (4.1)-(4.5) al resolver la ecuación de Enskog por medio del método de momentos de Grad, hemos tomado en cuenta un desarrollo del término colisional hasta segundo orden en el diámetro molecular, lo que conduce a gradientes de los flujos colisionales en la misma aproximación. Por tal motivo, y por razones de consistencia, las relaciones constitutivas deben contener flujos colisonales de orden 
superior. Las ecuaciones (2.15) y (2.19) se pueden escribir explícitamente, considerando la aproximación a segundo orden de los flujos colisionales en la representación a 13-momentos, en la forma siguiente:

$$
\begin{aligned}
\tilde{\stackrel{P}{P}}_{i j_{\text {total }}}(\vec{k}, \omega) & =\tilde{\tilde{P}}_{i j}^{K}(\vec{k}, \omega)+\tilde{\tilde{P}}_{i j}^{I}(\vec{k}, \omega)+\tilde{\tilde{P}}_{i j}^{I I}(\vec{k}, \omega) \\
& =\chi^{(2)} \tilde{\stackrel{P}{P}}_{i j}^{K}(\vec{k}, \omega)-\frac{6}{5} \tilde{\omega}\left[\left(i k_{i}, \tilde{u}_{j}\right)^{0}+\frac{\alpha}{10 \rho}\left(i k_{i} \tilde{q}_{j}^{K}\right)^{0}\right]
\end{aligned}
$$

y

$$
\begin{aligned}
\underset{\tilde{q}_{i}(\vec{k}, \omega)}{\text { total }} & =\tilde{q}_{i}^{K}(\vec{k}, \omega)+\tilde{q}_{i}^{I}(\vec{k}, \omega)+\tilde{q}_{i}^{I I}(\vec{k}, \omega) \\
& =\chi^{(3)} \tilde{q}_{i}^{K}-C_{v} \tilde{\omega}_{i} i k_{i} \tilde{T}-\frac{1}{2} \tilde{\omega}_{0}\left(i k_{i}, \tilde{P}_{i j}^{K}\right) .
\end{aligned}
$$

En estas expresiones hemos tomado en cuenta los resultados de las ecuaciones (2.67)-(2.70) para los flujos colisionales de primer y segundo orden en la densidad. Mediante una sustitución directa de estas ecuaciones en la relación formal (2.15) y (2.19) para el tensor de presiones total y el fujo de calor total obtenemos las relaciones entre flujos, ecuaciones (4.37)-(4.38), que toman en cuenta la contribución complementaria de la transferencia colisional. Como ya hemos mencionado, el mecanismo de transporte dominante depende de la densidad específica a la que se encuentra el gas de Enskog. Sin embargo, cualitativamente podemos decir que a bajas densidades la contribución cinética es dominante, a densidades moderadas las dos contribuciones son del mismo orden de magnitud, y a densidades elevadas las contribuciones colisionales determinan el transporte. Finalmente, cabe mencionar que las ecuaciones (4.37)-(4.38) son válidas en la región de vectores de onda y frecuencias finita. Las relaciones constitutivas completas se obtienen utilizando los operadores de proyección longitudinal y transversal en las ecuaciones (4.37) y (4.38), luego sustituyendo los flujos cinéticos de las ecuaciones (4.16)-(4.18) y reagrupando los términos de acuerdo con las diferentes variables conservadas, obtenemos, primero, la relación constitutiva del flujo de calor longitudinal total;

$$
\underset{\text { total }}{i k_{i} \tilde{q}_{i}(\vec{k}, \omega)}=\lambda(\vec{k}, \omega) k^{2} \tilde{T}-\Gamma_{1}(\vec{k}, \omega) k^{2}\left(i k_{i} \tilde{u}_{i}\right)
$$


donde la conductividad térmica generalizada, se puede expresar como:

$$
\begin{aligned}
& \lambda(\vec{k}, \omega)=\lambda_{0} \frac{\chi^{(3)^{2}}}{\chi} \cdot \Lambda_{\alpha, a d}^{K}(\vec{k}, \omega)+ \\
& +c_{v} \tilde{\omega}\left\{\left(1-i \omega \tau_{q}+\alpha^{(\lambda)} \tilde{\omega}_{0} \tau_{q} k^{2}\right)-\frac{4 k_{B} T}{9 m} \frac{\chi^{(3)^{2}} \tau_{p} \tau_{q} k^{2}}{\left(1-i \omega \tau_{p}+\frac{1}{2} \tilde{\omega}_{0} \tau_{p} k^{2}\right)}\right\} \Lambda_{a d, a d}^{K}(k, \omega)
\end{aligned}
$$

con,

$$
\alpha^{(\lambda)} \equiv\left[1-\frac{8}{45} \frac{\left(1-i \omega \tau_{q}+\tilde{\omega}_{0} \tau_{q} k^{2}\right)}{\left(1-i \omega \tau_{p}+\frac{1}{2} \tilde{\omega}_{0} \tau_{p} k^{2}\right)}\right]
$$

y el coeficiente cruzado $\Gamma_{1}$ se obtiene como:

$$
\begin{aligned}
& \Gamma_{1}(\vec{k}, \omega)=\frac{8}{9} \frac{\chi^{(3)} c_{v} T \tau_{p} \tau_{q}\left(\chi^{(2)} \chi^{(3)} n k_{B} T-\frac{1}{25} \cdot \frac{9}{4} \tilde{\omega} \tilde{\omega}_{0} k^{2}\right)}{\left(1-i \omega \tau_{q}+\tilde{\omega}_{0} \tau_{q} k^{2}\right)} \epsilon_{\alpha, a d}^{K}(\vec{k}, \omega) \\
& +\frac{8}{15} \chi^{(2)} c_{v} T \tilde{\omega} \tau_{p} \cdot \alpha^{\left(\gamma_{1}\right)} \cdot \epsilon_{\alpha, a d}^{K}(\vec{k}, \omega),
\end{aligned}
$$

con,

$$
\alpha^{\left(\gamma_{1}\right)} \equiv\left[1+\frac{3 \cdot 9}{8 \cdot 4} \frac{\chi^{(3)}}{\chi^{(2)}} \frac{\left(1-i \omega \tau_{p}+\frac{1}{2} \tilde{\omega}_{0} \tau_{p} k^{2}\right)}{\left(1-i \omega \tau_{q}+\tilde{\omega}_{0} \tau_{q} k^{2}\right)}\right] .
$$

En segundo lugar, la relación constitutiva para la parte longitudinal del tensor de presiones total esta dada por,

$$
i k_{i} i k_{j} \stackrel{\tilde{\check{P}}}{i j}_{\text {total }}(\vec{k}, \omega)=\frac{4}{3} n m \nu_{\ell}(\vec{k}, \omega) k^{2}\left(i k_{i} \tilde{u}_{i}\right)+\Gamma_{2}(\vec{k}, \omega) k^{4} \frac{\tilde{T}}{T},
$$

donde la viscosidad cortante longitudinal generalizada es,

$$
\begin{aligned}
& \nu_{\ell}(\vec{k}, \omega)=\frac{\eta_{0}}{n m} \cdot \frac{\chi^{(2)^{2}}}{\chi} \cdot \epsilon_{\alpha, a d}^{K}(\vec{k}, \omega)+ \\
& +\frac{1}{2} \tilde{\omega}_{0}\left\{\left(1-i \omega \tau_{p}+\frac{1}{2} \alpha^{\left(\nu_{\ell}\right)} \tilde{\omega}_{0} \tau_{p} k^{2}\right)+\chi^{\left(\nu_{\ell}\right)} \frac{8 k_{B} T \chi^{(3)^{2}} \tau_{p} \tau_{q} k^{2}}{15 m\left(1-i \omega \tau_{q}+\tilde{\omega}_{0} \tau_{q} k^{2}\right)}\right\} \cdot \epsilon_{\alpha, a d}(\vec{k}, \omega),
\end{aligned}
$$

con,

$$
\alpha^{\left(\nu_{\ell}\right)} \equiv\left[1-\frac{9}{80} \frac{\left(1-i \omega \tau_{p}+\frac{1}{2} \tilde{\omega}_{0} \tau_{p} k^{2}\right)}{\left(1-i \omega \tau_{q}+\tilde{\omega}_{0} \tau_{q} k^{2}\right)}\right] ; \quad \chi^{\left(\nu_{\ell}\right)} \equiv\left[1-\frac{13}{16} \frac{\chi^{(2)}}{\chi^{(3)}}\right]
$$


el coeficiente cruzado $\Gamma_{2}$ se escribe como:

$\Gamma_{2}(\vec{k}, \omega)=$

$$
\begin{aligned}
& \frac{8}{9} \frac{\chi^{(3)} c_{v} T \tau_{p} \tau_{q}\left(\chi^{(2)} \chi^{(3)} n k_{B} T-\frac{1}{25} \tilde{\omega} \tilde{\omega}_{0} k^{2}\right)}{\left(1-i \omega \tau_{q}+\tilde{\omega}_{0} \tau_{q} k^{2}\right)} \epsilon_{\alpha_{, a d}}^{K}(\vec{k}, \omega) \\
& +\frac{8}{15} \chi^{(2)} c_{v} T \tilde{\omega} \tau_{p} \alpha^{\left(\gamma_{2}\right)} \cdot \epsilon_{\alpha, a d}^{K}(\vec{k}, \omega) .
\end{aligned}
$$

donde,

$$
\alpha^{\left(\gamma_{2}\right)} \equiv\left[1+\frac{3 \chi^{(3)}}{8 \chi^{(2)}} \frac{\left(1-i \omega \tau_{p}+\frac{1}{2} \tilde{\omega}_{0} \tau_{p} k^{2}\right)}{\left(1-i \omega \tau_{q}+\tilde{\omega}_{0} \tau_{q} k^{2}\right)}\right] .
$$

Por último, la relación constitutiva del tensor de presiones transversal total,

$$
\mathbb{Q}_{t} \cdot\left(i k_{j} \stackrel{\tilde{o}}{P}_{i j}(\vec{k}, \omega)\right)=n m \nu_{t}(\vec{k}, \omega) k^{2} \tilde{u}_{i_{t}}
$$

donde la viscosidad cortante transversal generalizada, esta dada por:

$$
\begin{aligned}
& \nu_{t}(\vec{k}, \omega)=\frac{\eta_{0}}{n m} \cdot \frac{\chi^{(2)^{2}}}{\chi} \cdot \epsilon_{t \alpha_{, a d}}^{K}(\vec{k}, \omega)+ \\
& +\frac{1}{2} \tilde{\omega}_{0}\left\{\left(1-i \omega \tau_{p}+\frac{3}{7} \alpha^{\left(\nu_{t}\right)} \tilde{\omega}_{0} \tau_{p} k^{2}\right)+\right. \\
& \left.+\chi^{\left(\nu_{t}\right)} \frac{2 k_{B} T}{5 m} \frac{\chi^{(3)^{2}} \tau_{p} \tau_{q} k^{2}}{\left(1-i \omega \tau_{q}+\frac{1}{3} \tilde{\omega}_{0} \tau_{q} k^{2}\right)}\right\} \cdot \epsilon_{t \alpha, a d}(\vec{k}, \omega),
\end{aligned}
$$

$\mathrm{y}$,

$$
\alpha^{\left(\nu_{t}\right)} \equiv\left[1+\frac{7}{160} \frac{\left(1-i \omega \tau_{p}+\frac{3}{7} \tilde{\omega}_{0} \tau_{p} k^{2}\right)}{\left(1-i \omega \tau_{q}+\frac{1}{3} \tilde{\omega}_{0} \tau_{q} k^{2}\right)}\right] ; \quad \chi^{\left(\nu_{t}\right)} \equiv\left[1-\frac{\chi^{(2)}}{2 \chi^{(3)}}\right]
$$

Las ecuaciones (4.39), (4.44) y (4.49) son las relaciones constitutivas para la componente longitudinal y transversal del tensor de presiones y del flujo de calor, las cuales involucran a los flujos colisionales de orden superior. Las inhomogeneidades espaciales aparecen como términos nuevos que son proporcionales al cuadrado del vector de onda. El primer sumando de los CTG es el término dominante para vectores de onda y frecuencias cerca del régimen 
hidrodinámico para todo valor de la densidad, mientras que para vectores de onda y frecuencias elevadias, el segundo sumando tiende a una constante respecto a $(k, \omega)$ que depende de la densidad. Esto hace que los coeficientes de transporte no desciendan a cero completamente, manteniéndose a una cierta altura sobre el eje horizontal con el aumento del vector de onda o la frecuencia. Como hemos visto en el capítulo anterior, este comportamiento es característico de los resultados de dinámica molecular [32]. La presencia de la viscosidad volumétrica usual en el segundo sumando de los coeficientes generalizados, lo hace en general dos órdenes de magnitud más pequeño que el primer sumando, el cual por cierto contiene contribuciones de orden superior en las inhomogeneidades espaciales, como ya hemos mencionado, caracterizadas por los términos que contienen potencias cuadráticas del vector de onda y que se reducen a los resultados obtenidos en el capítulo anterior. Estos últimos contienen los rasgos principales del comportamiento de los CTG respecto del vector de onda y la frecuencia cerca del régimen hidrodinámico y es el que se reduce a los correspondientes coeficientes generalizados del gas diluído en el límite de bajas densidades [38,39]. Con la discusión anterior queremos resaltar el hecho de que los coeficientes generalizados, aún cuando son funciones complicadas del vector de onda y la frecuencia, en realidad solamente contienen pequeñas correciones en relación a los coeficientes que solo incluyen flujos colisionales de primer orden.

En el límite hidrodinámico de longitud de onda larga y bajas frecuencias; $|\vec{k}| \rightarrow 0, \omega \rightarrow 0$, los coeficientes de transporte generalizados se reducen a:

$$
\begin{gathered}
\lim _{\substack{|\vec{k}| \rightarrow 0 \\
\omega \rightarrow 0}} \lambda(\vec{k}, \omega)=\lambda_{0} \frac{\chi^{(3)^{2}}}{\chi}+c_{v} \tilde{\omega}, \\
\lim _{\substack{|\vec{k}| \rightarrow 0 \\
\omega \rightarrow 0}} \Gamma_{2}(\vec{k}, \omega)=\frac{16 T}{45}\left[\frac{\chi^{(2)} \chi^{(3)^{2}}}{\chi} \tau_{q} \lambda_{0}+\chi^{(2)} c_{v} \tau_{q} \tilde{\omega}\right],
\end{gathered}
$$

$\mathrm{y}$

$$
\lim _{\substack{|\vec{k}| \rightarrow 0 \\ \omega \rightarrow 0}} \nu_{t}(\vec{k}, \omega)=\frac{\eta_{0}}{n m} \cdot \frac{\chi^{(2)^{2}}}{\chi}+\frac{3}{5} \frac{\tilde{\omega}}{n m} .
$$

En este límite recuperamos los coeficientes de transporte usuales que se obtienen mediante el método de Chapman-Enskog cuando se incluyen flujos colisionales de orden superior [44-45]. También el coeficiente de termostricción tiene una corrección adicional y sigue siendo diferente de cero como 
en el caso de primer orden. Aquí también, hemos resuelto las ecuaciones de movimiento para las variables no-conservadas, expresando el flujo de calor y el tensor de presiones (parte simétrica s/traza) en términos de los gradientes de las variables conservadas. Este procedimiento nos condujo a las relaciones constitutivas generalizadas y a la forma explícita de los coeficientes de transporte generalizados.

El comportamiento de los coeficientes generalizados con respecto al vector de onda y la frecuencia está determinado por los tiempos de relajación asociados a los flujos del gas. Estos tiempos regulan el comportamiento hidrodinámico generalizado del sistema, mostrando como se lleva a cabo la dinámica del gas en el régimen cinético de vectores de onda y frecuencias finito, y permitiendo obtener expresiones explícitas de los flujos colisionales en términos de las integrales de colisión molecular, sin necesidad cle incluir ningún parámetro ajeno al modelo cinético.

\subsection{Viscosidad Volumétrica generalizada}

En el régimen hidrodinámico se obtiene un coeficiente de viscosidad volumétrica, cuando se incluyen en la descripción flujos colisiones de orden superior $[44,45]$. Este coeficiente es de origen puramente colisional, presentándose en sistemas de alta densidad, donde los efectos de volumen excluido se manifiestan como una resistencia del fluido a compresiones o expansiones [64-66]. El método de momentos de Grad aplicado a la ecuación usual de Enskog, proporciona este coeficiente de transporte como función del vector de onda y la frecuencia más allá del régimen hidrodinámico, razón por la cual lo denominaremos viscosidad volumétrica generalizada [67-70].

Como ya hemos visto, el flujo completo de cantidad de movimiento se puede expresar en términos de dos contribuciones al tensor de presiones: flujos cinéticos y flujos colisionales. El tensor de presiones se puede escribir formalmente como:

$$
\begin{aligned}
P_{i j_{\text {total }}}(\vec{x}, t) & \cong\left(\stackrel{o}{P}_{i j}^{K}(\vec{x}, t)+\dot{\circ}_{i j}^{I}(\vec{x}, t)+\stackrel{\circ}{P}_{i j}^{I I}(\vec{x}, t)\right)+ \\
& +\frac{1}{3}\left(P_{r r}^{K}(\vec{x}, t)+P_{r r}^{I}(\vec{x}, t)+P_{r r}^{I I}(\vec{x}, t)\right) \delta_{i j} .
\end{aligned}
$$

Esta expresión se obtiene separando la parte simétrica sin traza y la componente diagonal de la ecuación (2.15). La viscosidad volumétrica solamente se 
obtiene cuando consideramos flujos de ímpetu molecular a segundo orden en la transferencia colisional, y se encuentra asociada con la relación constitutiva de la parte diagonal del tensor de presiones;

$$
\pi(\vec{x}, t) \cong \pi_{0}+\pi_{d} \cong \frac{1}{3}\left(P_{r r}^{K}(\vec{x}, t)+P_{r r}^{I}(\vec{x}, t)+P_{r r}^{I I}(\vec{x}, t)\right)
$$

Esta expresión se obtiene procediendo por analogía con gases diluidos, donde se relaciona la traza del tensor de esfuerzos con una cantidad intensiva, la cual se puede descomponer en dos contribuciones:

a) Una relacionada con la presión termodinámica local, correspondiente a la parte no-disipativa, $\pi_{0}$.

b) Otra relacionada con la parte disipativa del tensor de presiones, $\pi_{d}$.

La representación a 13-momentos de la última igualdad de la ecuación (4.56) se obtiene de la siguiente manera: La definición de temperatura local nos da,

$$
\frac{1}{3} P_{r r}^{K}(\vec{x}, t)=n(\vec{x}, t) k_{B} T(\vec{x}, t) .
$$

Utilizando la función de distribución ecuación (2.31) podemos evaluar los flujos colisionales, para obtener las ecuaciones (2.67) y (2.68), de las cuales al tomar la traza obtenemos,

$$
\begin{gathered}
\frac{1}{3} P_{r r}^{I}(\vec{x}, t)=n(\vec{x}, t) k_{B} T(\vec{x}, t) \chi b \rho . \\
\frac{1}{3} P_{r r}^{I I}(\vec{x}, t)=-\tilde{\omega}\left(\frac{\partial u_{i}}{\partial x_{i}}(\vec{x}, t)\right)-\frac{\tilde{\omega} \alpha}{10 \rho}\left(\frac{\partial q_{i}^{K}}{\partial x_{i}}(\vec{x}, t)\right) .
\end{gathered}
$$

donde los parámetros que aparecen tienen el mismo significado que en el Capítulo 3. En particular $\tilde{\omega}$ es la viscosidad volumétrica usual del régimen hidrodinámico.

Sustituyendo las ecuaciones (4.57)-(4.59) en la ecuación (4.56) se identificará,

$$
\pi_{0}(\vec{x}, t)=(1+\chi b \rho) n(\vec{x}, t) k_{B} T(\vec{x}, t),
$$

como la presión, que ahora contiene la contribución del primer término colisional, dando lugar a las correcciones de la ecuación de estado de gas ideal y que tienen su origen en el comportamiento del gas denso. 
Por otra parte, la parte disipativa es,

$$
\pi_{d}(\vec{x}, t)=-\tilde{\omega}\left(\frac{\partial u_{i}}{\partial x_{i}}(\vec{x}, t)\right)-\frac{\tilde{\omega} \dot{\alpha}}{10 \rho}\left(\frac{\partial q_{i}^{K}}{\partial x_{i}}(\vec{x}, t)\right) .
$$

Las variables relevantes están todavía expresadas como funciones de las coordenadas espaciales y del tiempo. Son precisamente los flujos colisionales de orden superior los que dan origen a una ecuación de relajamiento, ecuación (4.61). Esta última ecuación tiene la forma de una relación constitutiva generalizada, el primer sumando es el resultado que se obtiene en el régimen hidrodinámico al utilizar el método de Chapman-Enskog, mientras que el segundo proporciona las contribuciones en la región de vectores de onda y frecuencias finitas.

Vamos a realizar un proceso de reducción en la relación constitutiva de la ecuación (4.61). Para ello, consideramos su forma linealizada que describe la evolución de perturbaciones pequeñas alrededor del estado de equilibrio, de donde después de aplicar la transformada de Fourier correspondiente, obtenemos:

$$
\tilde{\pi}_{d}=-\tilde{\omega}\left(i k_{i} \tilde{u}_{i}\right)-\frac{\tilde{\omega}}{10 \rho \alpha}\left(i k_{i} \tilde{q}_{i}^{K}\right) .
$$

Las cantidades con tilde son funciones del vector de onda y la frecuencia. Sustituyendo en esta ecuación la parte cinética longitudinal del flujo de calor, ecuación (4.16) y separando los términos proporcionales a cada una de las variables relevantes conservadas, podemos reescribir la ecuación (4.62) en la forma de una relación constitutiva generalizadia, en el espacio $(\vec{k}, \omega)$

$$
\tilde{\pi}_{d}(\vec{k}, \omega)=-\tilde{\omega}(\vec{k}, \omega)\left(i k_{i} \tilde{u}_{i}\right)-\Gamma_{d}(\vec{k}, \omega) k^{2} \tilde{T}
$$

donde la viscosidad volumétrica generalizada (VVG), es el coeficiente de la cantidad $i k_{i} \tilde{u}_{i}$ y se identifica como:

$$
\begin{aligned}
& \tilde{\omega}(\vec{k}, \omega)=\tilde{\omega}\left\{\left(1-i \omega \tau_{q}+\frac{77}{80} \tilde{\omega}_{0} \tau_{q} k^{2}\right)+\right. \\
& \left.\chi^{(\pi)} \frac{8 k_{B} T}{15 m} \chi^{(3)^{2}} \frac{\tau_{p} \tau_{q} k^{2}}{\left(1-i \omega \tau_{p}+\frac{1}{2} \tilde{\omega}_{0} \tau_{p} k^{2}\right)}\right\} \cdot \Lambda_{\alpha, a d}^{K}(\vec{k}, \omega),
\end{aligned}
$$

con,

$$
\chi^{(\pi)}(\rho)=\left[1-\frac{\chi^{(2)}}{4 \chi^{(3)}}\right]
$$


El coeficiente térmico de disipasión, $\Gamma_{d} \mathrm{es}$,

$$
\Gamma_{d}(\vec{k}, \omega)=\frac{1}{6} \chi^{(3)} c_{v} \tilde{\omega} \tau_{q} \alpha^{(\tilde{\omega})}(\vec{k}, \omega) \cdot \Lambda_{\alpha, a d}^{K}(\vec{k}, \omega)
$$

donde

$$
\alpha^{(\tilde{\omega})}(\vec{k}, \omega)=\left[1-\frac{4}{15} \frac{\tilde{\omega}_{0} \tau_{p} k^{2}}{\left(1-i \omega \tau_{p}+\frac{1}{2} \tilde{\omega}_{0} \tau_{p} k^{2}\right)}\right]
$$

y nos mide el efecto de las inhomogeneidades en la temperatura sobre el flujo de ímpetu en el sistema.

La ecuación (4.61) muestra que la traza del tensor de presiones contiene una contribución a la fuerza termodinámica usual que da origen a la viscosidad volumétrica, así como un término proporcional al flujo cle calor el cual da origen a los términos disipativos en la región $(\vec{k}, \omega)$-finita de la relación constitutiva generalizada. Notemos que también en este caso los tiempos de relajamiento de los flujos determinan la región de las inhomogeneidades espaciales de orden superior [71-73] son importantes.

En las gráficas, la viscosidad volumétrica generalizada y el coeficiente térmico $\Gamma_{d}$ generalizado están normalizados con respecto a sus valores en el régimen hidrodinámico, de tal manera que toda la dependencia en la densidad se ha dejado del lado derecho de sus expresiones en las ecuaciones (4.64) y (4.66). Solamente hemos considerado el caso $\omega=0$ debido a que la dependencia en la frecuencia es irrelevante a vector de oncla cero; en efecto, la viscosidad volumétrica es la constante $\tilde{\omega}$, mientras que el coeficiente térmico tiene el comportamiento similar al de los otros coeficientes generalizados y que ya hemos tratados anteriormente.

El comportamiento de $\chi^{(\pi)}(\rho)$, dado en la ec.(4.65), con respecto a la densidad se muestra en la figura 7 . Vemos que es una función creciente a bajas densidades y que se satura completamente hacia un valor asintótico menor que uno, esto es muy importante porque asegura un descenso para la curva de la viscosidad volumétrica que se obtiene de la ecuación (4.64). Más aún, la viscosidad volumétrica desciende casi completamente en $\kappa \sim 2$, para una densidad $\delta_{0}=0.144$ típica del gas denso, el mismo valor al que decaen los otros coeficientes generalizados. Para valores de $\kappa \geq 2.5$ la viscosidad volumétrica permanece a una cierta altura sin anularse, determinada por $\chi^{(\pi)}$. Lo anterior se debe a que este coeficiente de transporte proviene de las contribuciones a los flujos por transferencia colisional, los cuales solo 


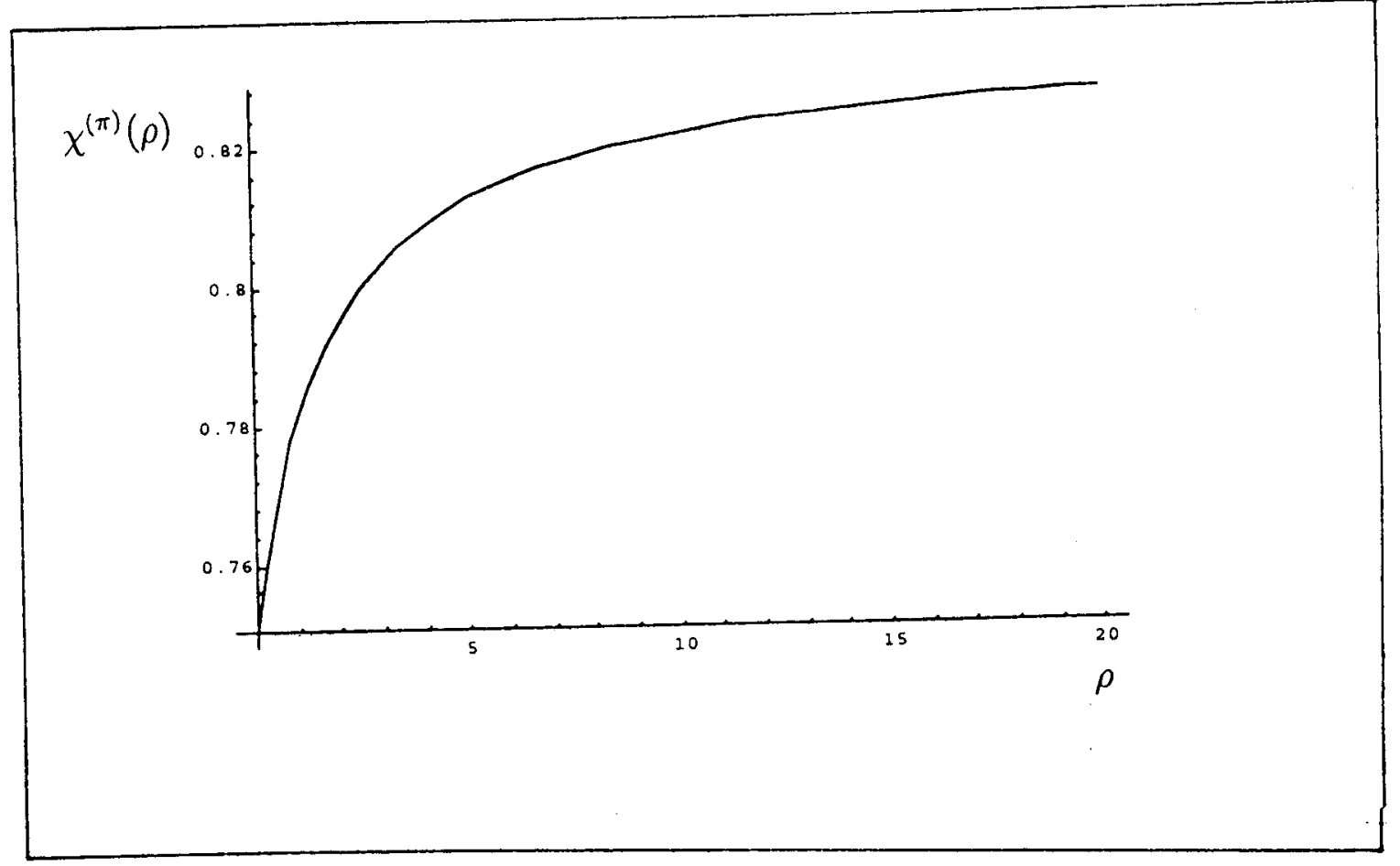

Figure 7: Factor densidad que regula el comportamiento de la VVG, ecuación (4.65), que independientemente de la densidad siempre es menor que la unidad. 
son significativos a densidades elevadas donde siempre existen correlaciones espaciales.

Como ya hemos visto anteriormente, la incorporación de flujos colisionales en las relaciones constitutivas generalizadas, amortigua el clescenso de los coeficientes de transporte haciendo las curvas más planas conforme aumenta la densidad. Esta característica se manifiesta más significativamente en la viscosidad volumétrica, puesto que la curva ya no tiende más a cero, permaneciendo a una cierta altura, independientemente del vector de onda.

En la figura 8 también se muestra el comportamiento del coeficiente térmico $\Gamma_{d}$, con respecto al vector de onda, observamos que tiende muy rápido a cero, de tal forma que para $\kappa \sim 3.0$ su valor es un orden de magnitud más pequeño. Su comportamiento en vectores de oncla y frecuencias es cualitativamente similar al de los coeficientes cruzados generalizados. El comportamiento general de las curvas de los coeficientes de transporte generalizados con variaciones en la frecuencia es igual, al menos cualitativamente, al que tienen algunas funciones de memoria asociadas con las funciones de correlación correspondientes. Esto es de esperarse puesto que estos coeficientes, como veremos en el Capítulo 5 son los elementos de una matriz de memoria en el esquema de la ecuación de Langevin generalizada.

Podemos considerar los límites del régimen hidrodinámico en longitudes de onda larga y bajas frecuencias;

$$
\begin{gathered}
\lim _{\substack{|\vec{k}| \rightarrow 0 \\
\omega \rightarrow 0}} \tilde{\omega}(\vec{k}, \omega)=\tilde{\omega}, \\
\lim _{\substack{|\vec{k}| \rightarrow 0 \\
\omega \rightarrow 0}} \Gamma_{d}(\vec{k}, \omega)=\frac{1}{6} \chi^{(3)} c_{v} \tilde{\omega} \tau_{q},
\end{gathered}
$$

esto debido a que el límite correspondiente de $\alpha^{(\tilde{\omega})}(\vec{k}, \omega)$ es la unidad. En el régimen hidrodinámico recuperamos la viscosidad volumétrica usual, que se obtiene mediante el método de Chapman-Enskog. Su valor numérico expresado en términos de las integrales de colisión molecular corresponde a la ecuación (2.65).

Regresando al tensor de presiones de la ecuación (4.55) podemos escribirlo en la forma de una relación constitutiva con ayuda de las ecuaciónes (4.44) 


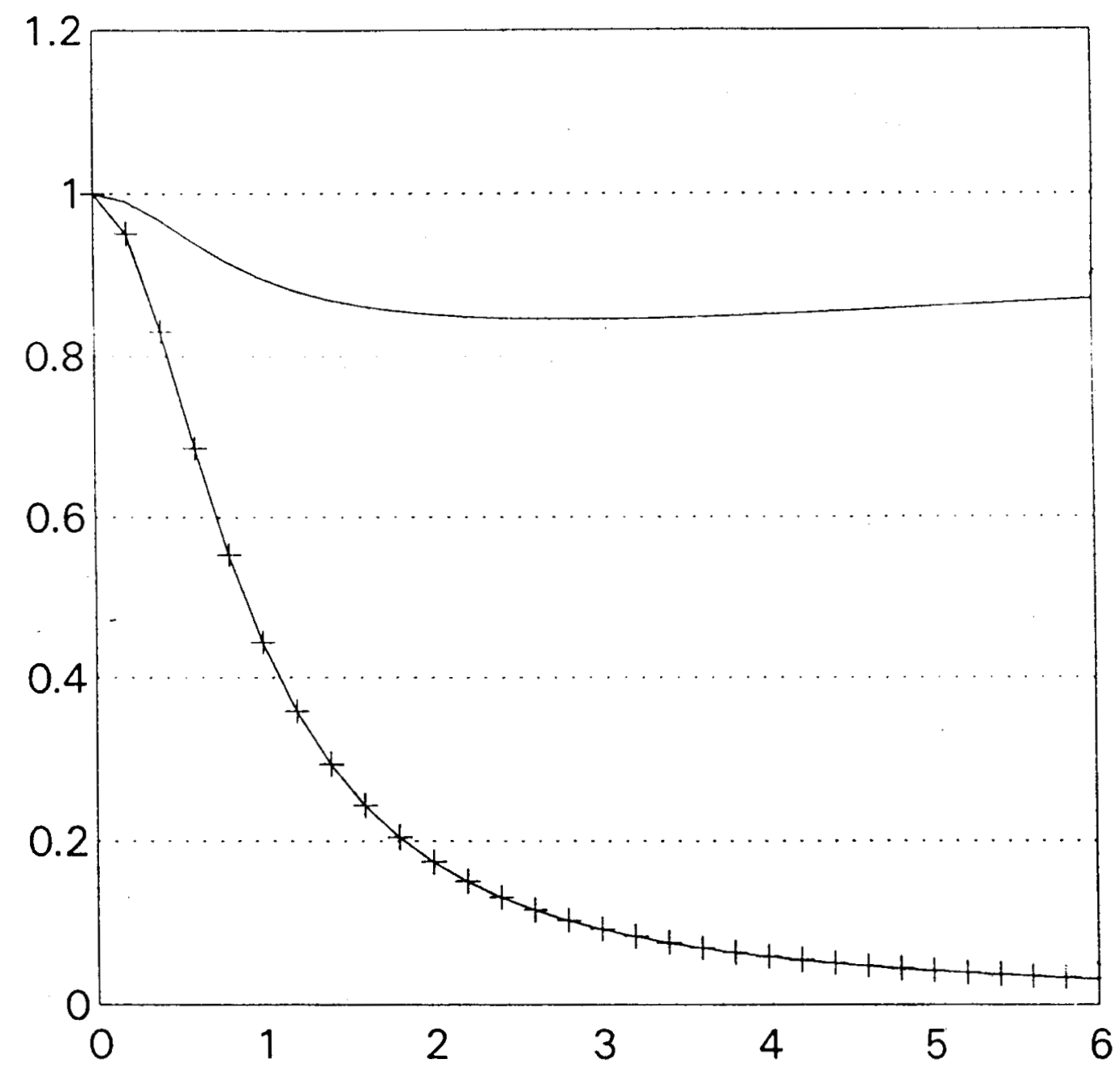

Figure 8: Viscosidad volumétrica generalizadla (la curva continua), el coeficiente térmico generalizado (la curva con cruces) como función de $k a$ a una densidad reducida específica de 0.144 . 
y (4.63). Tomando la proyección longitudinal de esta ecuación,

$$
\begin{aligned}
i k_{i} i k_{j} \tilde{P}_{i j}(\vec{k}, \omega) \cong & i k_{i} i k_{j} \stackrel{\circ}{P}_{i j}(\vec{k}, \omega)+\tilde{\pi}(\vec{k}, \omega)(i k)^{2} \delta_{i j} \\
= & \left(\frac{4}{3} n m \nu_{\ell}(\vec{k}, \omega)+\tilde{\omega}(\vec{k}, \omega)\right) k^{2}\left(i k_{i} \tilde{u}_{i}\right) \\
& +\left(\Gamma_{2}(\tilde{k}, \omega)+\Gamma_{d}(\vec{k}, \omega) T\right) k^{4} \frac{\tilde{T}}{T}
\end{aligned}
$$

reescribiendo además, (4.39) obtenemos:

$$
i k_{i} \tilde{q}_{i}(\vec{k}, \omega) \cong \lambda(\vec{k}, \omega) k^{2} \tilde{T}-\Gamma_{1}(\vec{k}, \omega) k^{2}\left(i k_{i} \tilde{u}_{i}\right)
$$

para fines de referencia y comparación de su estructura, nos encontramos con que las ecuaciones anteriores describen un efecto cruzado a través de los coeficientes $\Gamma$-as.

En gases diluidos los coeficientes de transporte que describen este efecto cruzado son iguales, y lo mismo sucede cuando consideramos los flujos colisionales a primer orden en el modelo cinético de Enskog [62]. En este caso, podemos hacer una comparación directa de los efectos cruzados, utilizando las expresiones explícitas de los coeficientes generalizados de las ecuaciones (4.42), (4.47) y (4.66), de donde resulta (ver apéndice C):

$$
\Gamma_{1}(\vec{k}, \omega)=\Gamma_{2}(\vec{k}, \omega)+\Gamma_{d} T .
$$

Por lo tanto los coeficientes de transporte cruzados, en las relaciones constitutivas generalizadas de un gas de Enskog, son iguales dentro del modelo cinético que incluye flujos colisionales a segundo orden en los gradientes. 


\section{Capítulo 5}

\section{Hidrodinámica Generalizada}

En este capítulo utilizamos los resultados del modelo cinético de Grad-Enskog, que se ha desarrollado anteriormente, para construir la descripción usual de la hidrodinámica generalizada. En particular, estamos interesados en encontrar la relación que existe entre los coeficientes de transporte generalizados y las funciones de memoria que describen los efectos no-locales y no-instantáneos del sistema. Para introducir la matriz de memoria recurrimos a una ecuación de Langevin fenomenológica que proviene de una generalización intuitiva de las ecuaciones de movimiento para una partícula Browniana.

\subsection{Ecuación de Langevin Generalizada}

En la teoría general de las funciones de correlación, la evolución temporal de las fluctuaciones requiere de las ecuaciones de movimiento que gobiernan el comportamiento de las variables dinámicas relevantes. Existen tratamientos tanto microscópicos exactos, como macroscópicos aproximados para describir la evolución temporal de las variables relevantes y de sus fluctuaciones. Desde el punto de vista microscópico el principal problema que se presenta, consiste básicamente en que se deben tomar en cuenta las propiedades dinámicas de un sistema de muchos cuerpos. Los tratamientos que parten de principios fundamentales son capaces de abordar rigurosamente, además de cuestiones tan importantes como la irreversibilidad, el problema de obtener las ecuaciones macroscópicas junto con los coeficientes de transporte correspondientes. Esto a partir de las ecuaciones microscópicas que gobiernan el movimiento de las partículas individuales del sistema [57]. No obstante, otros tratamientos menos rigurosos de carácter fenomenológico utilizan ecuaciones de tipo hidrodinámico, complementadas con coeficientes de transporte y variables termodinámicas dados por fuera del modelo a través de datos experimentales o mediante algún otro esquema. En lo que sigue de este capítulo vamos a considerar un acercamiento de los resultados de la teoría cinética, precisamente con uno de estos últimos tratamientos el cual vamos a considerar más esfecíficamente.

Una separación en las ecuaciones de evolución macroscópicas, que des- 
criben la hidrodinámica de un fluido simple, se lleva a cabo con respecto al intervalo de vectores de onda y frecuencias que se están considerando. En el régimen hidrodinámico, de longitudes de onda larga y bajas frecuencias, se pueden utilizar las ecuaciones de Navier-Stokes para describir aproximadamente, el decaimiento a tiempos largos de las funciones de correlación. Por otro lado, en los procesos de relajación y transporte que ocurren en la región de dinámica molecular se puede considerar un tratamiento fenomenológico, en base a la ecuación de Langevin del movimiento Browniano, muy utilizado para estudiar el comportamiento de las funciones de correlación en la región de vectores de onda grandes y frecuencia altas.

Recordemos las principales características de la teoría estocástica de Langevin;

i) La ecuación del movimiento de una partícula Browniana de masa $m$ y velocidad $u_{i}(t)$,

$$
m \frac{d u_{i}(t)}{d t}=-m \int_{0}^{t} \xi\left(t-t^{\prime}\right) u_{i}\left(t^{\prime}\right) d t^{\prime}+R_{i}(t)
$$

siendo $\xi(t)$ la llamada función de memoria o coeficiente de fricción generalizado, mientras que $R_{i}(t)$ es la fuerza aleatoria fluctuante que proviene de las colisiones de la partícula con la gran cantidad de moléculas que la rodean.

ii) La fuerza fluctuante $R_{i}(t)$ se anula en promedio, es decir,

$$
\left\langle R_{i}(t)\right\rangle=0
$$

los paréntesis angulares indican un promedio sobre algún ensemble representativo.

iii) La velocidad de la partícula Browniana no está correlacionada con la fuerza fluctuante que aparece a tiempos posteriores, esto es,

$$
\left\langle R_{i}(t) u_{i}(0)\right\rangle=0
$$

Estos resultados pueden generalizarse a variables dinámicas arbitrarias, a través de argumentos intuitivos sobre el significado del coeficiente de fricción y de la fuerza aleatoria. Desde este punto de vista, la descripción de un 
estado fuera de equilibrio en fluidos simples se efectúa en términos de un conjunto de variables relevantes $A_{i}(t)$, las cuales se supone evolucionan en el tiempo de acuerdo con la "ecuación de Langevin generalizadla", que se puede escribir como sigue,

$$
\begin{aligned}
\frac{\partial A_{i}(\vec{k}, t)}{\partial t} & =i \Omega_{i j}(\vec{k}) A_{j}(\vec{k}, t)-\int_{0}^{t} K_{i j}\left(\vec{k}, t^{\prime}\right) A_{j}\left(\vec{k}, t-t^{\prime}\right) d t^{\prime} \\
& +f_{i}(\vec{k}, t)
\end{aligned}
$$

donde $\Omega_{i j}(\vec{k})$ se denomina matriz frecuencia, mientras que $K_{i j}(\vec{k}, t)$ es la matriz de memoria y $f_{i}(\vec{k}, t)$ es la llamada fuerza fluctuante, asociada con cada variable relevante $[1,7]$. Esta ecuación generaliza el carácter no-markoffiano del movimiento Browniano a otras variables dinámicas.

La importancia práctica de la ec. (5.1), radica en que un proceso dinámico complicado se puede abordar fenomenológicamente utilizando funciones de memoria, más o menos sencillas, cuyos parámetros libres se ajustan pidiendo que se cumplan las bien conocidas reglas de suma de la frecuencia. Sin embargo, es muy importante distinguirla claramente, de la ecuación de Langevin generalizada (GLE) que se obtiene rigurosamente en el esquema de Zwanzig-Mori en la descripción de fenómenos microscópicos fundamentales de la Mecánica Estadística fuera de equilibrio. En este contexto la GLE contiene toda la dinámica del sistema, siendo equivalente a la ecuación de Liouville de las variables dinámicas relevantes, por tal motivo se requiere todavía de un mayor análisis y de introducir algunas suposiciones adicionales antes de identificarla como una ecuación diferencial estocástica [75].

Si las variables $A_{i}(\vec{k}, t)$ se eligen de manera que representen los procesos cle relajación lenta en el fluido, entonces la ecuación de Langevin generalizada, ec. (5.1), es una buena descripción del comportamiento hidrodinámico del sistema en la región de vectores de onda grande y frecuencias altias. La matriz frecuencia determina, a través de sus eigenvalores, las frecuencias de los modos colectivos de relajación lenta. La matriz de amortiguamiento, o kernel de memoria $K_{i j}\left(\vec{k}, t^{\prime}\right)$, como su nombre lo indica nos dará las constantes de amortiguamiento, cantidades estrechamente relacionadas con los coeficientes de transporte generalizados. En este caso las variables del sistema, evolucionan de acuerdo con el promedio de la ec.(5.1) lo que nos conduce a que la fuerza aleatoria $f_{i}(\vec{k}, t)$ sea una cantidad irrelevante. 


\subsection{Funciones de Memoria}

El carácter fenomenológico de la ecuación de Langevin generalizadla, ec. (5.1), nos impide tener más información sobre la matriz frecuencia y la matriz de memoria. Para superar esta dificultad, lo ideal es contar con un tratamiento microscópico que permita obtener explícitamente los elementos de dichas matrices de manera más sistemática. En esta sección vamos a expresar las ecuaciones hidrodinámicas linealizadas del tipo Navier-Stokes, que resultan del modelo cinético Grad-Enskog, como una ecuación matricial de Langevin generalizada.

El procedimiento mencionado anteriormente nos pone en contacto con los tratamientos usuales de la hidrodinámica generalizada y consiste, principalmente, en expresar las ecuaciones de movimiento de las variables relevantes en el espacio de Fourier, para luego reducir la descripción del modelo cinético a la representación de variables conservadas e identificar a la matriz frecuencia y a la matriz de memoria, en base a ciertas propiedades generales que deben satisfacer.

El método de Grad en la aproximación de 13-momentos, proporciona las ecuaciones de evolución para las variables relevantes conservadas de un gas denso de Enskog a segundo orden en los gradientes de flujos colisionales. Las ecuaciones (2.71)-(2.73) para $\rho(\vec{x}, t), u_{i}(\vec{x}, t), T(\vec{x}, t)$ son,

$$
\begin{gathered}
\frac{\partial \rho}{\partial t}+\rho \frac{\partial u_{i}}{\partial x_{i}}=0 \\
\rho \frac{\partial u_{i}}{\partial t}+\frac{\chi^{(\rho)}}{\alpha} \frac{\partial \rho}{\partial x_{i}}-\rho \tilde{\omega}_{0}\left\{\frac{1}{2} \frac{\partial^{2} u_{i}}{\partial x_{k} \partial x_{k}}+\frac{\partial^{2} u_{k}}{\partial x_{k} \partial x_{i}}\right\}+ \\
+\frac{\rho}{\alpha T} \chi^{(5)} \frac{\partial T}{\partial x_{i}}+\chi^{(2)} \frac{\partial}{\partial x_{j}} \stackrel{\circ}{P}_{i j}^{K}-\frac{\alpha \tilde{\omega}_{0}}{10}\left\{\frac{1}{2} \frac{\partial^{2} q_{i}^{K}}{\partial x_{k} \partial x_{k}}+\frac{\partial^{2} q_{k}^{K}}{\partial x_{k} \partial x_{i}}\right\}=0 \\
\frac{3}{2} \frac{\rho}{\alpha T} \frac{\partial T}{\partial t}+\chi^{(3)} \frac{\partial q_{k}^{K}}{\partial x_{k}}-C_{\imath} \tilde{\omega} \frac{\partial^{2} T}{\partial x_{k} \partial x_{k}}-\frac{1}{2} \tilde{\omega}_{0} \frac{\partial^{2} \stackrel{P}{k}_{k \ell}^{K}}{\partial x_{k} \partial x_{\ell}}+ \\
+\frac{\rho}{\alpha} \chi^{(5)} \frac{\partial u_{k}}{\partial x_{k}}=0
\end{gathered}
$$


recordemos las definiciones,

$$
\begin{aligned}
\chi^{(\rho)} & \equiv\left(1+2 \chi b p+\frac{\partial \chi}{\partial \rho} b \rho^{2}\right) \\
\alpha & =\frac{m}{k_{B} T}, \quad \frac{\rho}{\alpha T}=n k_{B}, \quad \alpha \tilde{\omega}_{0}=\frac{6}{5} \frac{\tilde{\omega}}{n k_{B} T} .
\end{aligned}
$$

Pasando estas ecuaciones al espacio $(\vec{k}, \omega)$ de Fourier, las podemos escribir como,

$$
\begin{gathered}
i \omega \tilde{\rho}+\rho\left(i k_{i} \tilde{u}_{i}\right)=0, \\
-i \omega \rho \tilde{u}_{i}+\frac{\chi^{(\rho)}}{\alpha} i k_{i} \tilde{\rho}-\rho \tilde{\omega}_{0}\left\{\frac{1}{2}(i k)^{2} \tilde{u}_{i}+i k_{i}\left(i k_{j} \tilde{u}_{j}\right)\right\}+\frac{\rho}{\alpha T} \chi^{(5)} i k_{i} \tilde{T}+ \\
+\chi^{(2)} i k_{j} \tilde{\stackrel{\rho}{P}}_{i j}^{K}-\frac{\alpha \tilde{\omega}_{0}}{10}\left\{\frac{1}{2}(i k)^{2} \tilde{q}_{i}^{K}+i k_{i}\left(i k_{j} \tilde{q}_{j}^{K}\right)\right\}=0, \\
-i \omega \cdot \frac{3}{2} \frac{\rho}{\alpha T} \tilde{T}+\chi^{(3)}\left(i k_{i} \tilde{q}_{i}^{K}\right)-c_{v} \tilde{\omega}(i k)^{2} \tilde{T}-\frac{1}{2} \tilde{\omega}_{0}\left(i k_{i} i k_{j} \tilde{\stackrel{\rho}{P}}_{i j}^{K}\right) \\
\frac{\rho}{\alpha} \chi^{(5)}\left(i k_{i} \tilde{u}_{i}\right)=0 .
\end{gathered}
$$

La componente longitudinal de estas ecuaciones ya la hemos considerado anteriormente, ecuaciones (4.8)-(4.10), y las vamos a escribir como sigue;

$$
\begin{gathered}
-i \omega \tilde{\rho}=-\rho\left(i k_{i} \tilde{u}_{i}\right), \\
-i \omega \rho\left(i k_{i} \tilde{u}_{i}\right)=-\frac{\chi^{(\rho)}}{\alpha}(i k)^{2} \tilde{\rho}+\rho \tilde{\omega}_{0}\left\{\frac{3}{2}(i k)^{2}\left(i k_{i} \tilde{u}_{i}\right)\right\}- \\
-\frac{\rho}{\alpha T} \chi^{(5)}(i k) \tilde{T}-\chi^{2}\left(i k_{i} i k_{j} \tilde{\tilde{\rho}}_{i j}^{K}\right)+\frac{\alpha \tilde{\omega}_{0}}{10}\left\{\frac{3}{2}(i k)^{2}\left(i k_{i} \tilde{q}_{i}^{K}\right)\right\} \\
-i \omega \cdot \frac{3}{2} \frac{\rho}{\alpha T} \tilde{T}=-\chi^{(3)}\left(i k_{i} \tilde{q}_{i}^{K}\right)+c_{v} \tilde{\omega}(i k)^{2} \tilde{T}+\frac{1}{2} \tilde{\omega}_{0}\left(i k_{i} i k_{j} \tilde{\stackrel{P}{P}}_{i j}^{K}\right) \\
\frac{\rho}{\alpha} \chi^{(5)}\left(i k_{i} \tilde{u}_{i}\right) .
\end{gathered}
$$


Estas ecuaciones las podemos simplificar con ayuda de las relaciones;

$$
\begin{aligned}
& i k_{i} i k_{j} \tilde{\tilde{P}}_{i j}=\left(i k_{i} i k_{j} \tilde{P}_{i j}^{K}\right)-\frac{2}{3} \rho \tilde{\omega}_{0}(i k)^{2}\left(i k_{i} \tilde{u}_{i}\right)- \\
&-\frac{2}{3} \cdot \frac{\alpha \tilde{\omega}_{0}}{10}(i k)^{2}\left(i k_{i} \tilde{q}_{i}^{K}\right), \\
&(i k)^{2} \pi_{d}=\left(i k_{i} i k_{j}\right) \pi_{d} \delta_{i j}= \\
&=-\frac{5}{6} \rho \tilde{\omega}_{0}(i k)^{2}\left(i k_{i} \tilde{u}_{i}\right)-\frac{5}{6} \frac{\alpha \tilde{\omega}_{0}}{10}(i k)^{2}\left(i k_{i} \tilde{q}_{i}^{K}\right), \\
& i k_{i} \tilde{q}_{i}=\chi^{(3)}\left(i k_{i} \tilde{q}_{i}^{K}\right)-C_{v} \tilde{\omega}(i k)^{2} \tilde{T}-\frac{1}{2} \tilde{\omega}_{0}\left(i k_{i} i k_{j} \tilde{P}_{i j}^{K}\right),
\end{aligned}
$$

ya que hemos identificado a los flujos completos de ímpetu y energía, es decir, al tensor cle presiones y al flujo de calor completos en su representación a 13-momentos y a segundo orden en los flujos colisionales.

Bajo estas condiciones las ecuaciones de conservación longitudinales se pueden expresar como:

$$
\begin{gathered}
-i \omega \tilde{\rho}=-\rho\left(i k_{i} \tilde{u}_{i}\right), \\
-i \omega \rho\left(i k_{i} \tilde{u}_{i}\right)=-\frac{\chi^{(\rho)}}{\alpha}(i k)^{2} \tilde{\rho}-\left(i k_{i} i k_{j} \tilde{P}_{i j}\right)-(i k)^{2} \pi_{d}- \\
-\frac{\rho}{\alpha T} \chi^{(5)}(i k)^{2} \tilde{T}, \\
-i \omega \cdot \frac{3}{2} \frac{\rho}{\alpha T} \tilde{T}=-\left(i k_{i} \tilde{q}_{i}\right)-\frac{\rho}{\alpha} \chi^{(5)}\left(i k_{i} \tilde{u}_{i}\right) .
\end{gathered}
$$

Para obtener un conjunto cerrado de ecuaciones de balance, vamos a seguir un procedimiento similar al que se hace para obtener las ecuaciones de la hidrodinámica usual de Navier-Stokes, a saber, utilizar el conjunto de relaciones constitutivas de Navier-Newton-Fourier, para expresar los flujos en términos de los gradientes de las variables conservadas.

En nuestro caso vamos a utilizar las relaciones constitutivas generalizadas obtenidas anteriormente, ecuaciones (4.39), (4.44) y (4.63) las cuales vamos a modificar ligeramente en notación simplificando la dependencia en vectores de onda y frecuencia, la cual se sobreentiende, y redefiniendo el 
último coeficiente $\Gamma$ para tener una notación homogénea:

$$
\begin{aligned}
i k_{i} \tilde{q}_{i} & =\lambda(\vec{k}, \omega) k^{2} \tilde{T}-\Gamma_{1}(\vec{k}, \omega) k^{2}\left(i k_{i} \tilde{u}_{i}\right) \\
& \doteq \lambda k^{2} \tilde{T}-\Gamma_{1} k^{2}\left(i k \tilde{u}_{\ell}\right), \\
i k_{i} i k_{j} \tilde{\hat{P}}_{i j} & =\frac{4}{3} n m \nu_{\ell}(\vec{k}, \omega) k^{2}\left(i k_{i} \tilde{u}_{i}\right)+\Gamma_{2}(\vec{k}, \omega) \vec{k}^{4} \frac{\tilde{T}}{T} \\
& \hat{=} n m \dot{\nu}_{\ell}\left(i k \tilde{u}_{\ell}\right)+\Gamma_{2} k^{4} \frac{\tilde{T}}{T}, \\
\pi_{d} & =-\tilde{\omega}(\vec{k}, \omega)\left(i k_{i} \tilde{u}_{i}\right)-\Gamma_{d}(\vec{k}, \omega) k^{2} \tilde{T} \\
& \hat{=}-n m \tilde{\omega}_{g}\left(i k \tilde{u}_{\ell}\right)-\Gamma_{g} k^{2} \frac{\tilde{T}}{T} .
\end{aligned}
$$

El simbolo $(\hat{=})$ significa que la igtaldad incluye definiciones y cambios de notación. Sustituyendo estas relaciones constitutivas generalizadas en las ecuaciones de conservación, ecuaciones (5.15)-(5.17), obtenemos un conjunto de ecuaciones hidrodinámicas con coeficientes de transporte generalizados $[38,62]$. Expresando el resultado en términos de las variables adimensionales,

$$
\left(\tilde{n}^{*}=\frac{\tilde{n}}{n}, \quad \tilde{u}_{\ell}^{*}=\left(\frac{\alpha}{\chi^{(\rho)}}\right)^{1 / 2} \tilde{u}_{\ell}, \quad \tilde{T}^{*}=\left(\frac{3}{2 \chi^{(\rho)}}\right)^{1 / 2} \frac{\tilde{T}}{T}\right)
$$

se tiene

$$
\begin{gathered}
-i \omega \tilde{n}^{*}=-i k\left(\frac{\chi^{(\rho)}}{\alpha}\right)^{1 / 2} \tilde{u}_{\ell}^{*} \\
-i \omega \tilde{u}_{\ell}^{*}=-i k\left(\frac{\chi^{(\rho)}}{\alpha}\right)^{1 / 2} \tilde{n}^{*}-\chi^{(5)}\left(\frac{2}{3 \alpha}\right)(i k) \tilde{T}^{*}- \\
-\left(\dot{\nu}_{\ell}+\tilde{\omega}_{g}\right) k^{2} \tilde{u}_{\ell}^{*}+\frac{\left(\Gamma_{2}+\Gamma_{g}\right)}{n k_{B} T}\left(\frac{2}{3 \alpha}\right)^{1 / 2} i k^{3} \tilde{T}^{*}, \\
-i \omega \tilde{T}^{*}=-\chi^{(5)}\left(\frac{2}{3 \alpha}\right)^{1 / 2}(i k) \tilde{u}_{\ell}^{*}-\frac{2 \lambda}{3 n k_{B}} k^{2} \tilde{T}^{*}+ \\
+\frac{\Gamma_{1}}{n k_{B} T}\left(\frac{2}{3 \alpha}\right)^{1 / 2} i k^{3} \tilde{u}_{\ell}^{*} .
\end{gathered}
$$


Formalmente estas expresiones coinciden con las que se obtienen en la aproximación a primer orden en los flujos colisionales. Sin embargo, las expresiones para los coeficientes de transporte generalizados son mucho más complicadas en las ecuaciones (5.22)-(5.24) debido a la contribución de flujos colisionales de orden superior. Debemos subrayar la presencia de la viscosidad volumétrica generalizada en la ecuación de movimiento del gas, la cual solo aparece cuando incorporamos flujos colisionales de segundo orden en los gradientes.

La forma matricial de estas ecuaciones es,

$$
-i \omega \tilde{A}_{i}(\vec{k}, \omega)=\mathcal{L}_{i j}(\vec{k}, \omega) \tilde{A}_{j}(\vec{k}, \omega),
$$

que corresponde a la forma general que se obtiene a partir de la transformada de Fourier de la ecuación (5.1). En nuestra descripción la elección de las variables relevantes conservadlas que definen el sistema, se agrupan en el vector columna $\tilde{A}_{i}$,

$$
\tilde{A}_{i}=\left(\begin{array}{c}
\tilde{n}^{*} \\
\tilde{u}_{\ell}^{*} \\
\tilde{T}^{*}
\end{array}\right)
$$

mientras que la matriz de coeficientes $\mathcal{L}_{i j}(\vec{k}, \omega)$ tiene la forma;

$$
\mathcal{L}_{i j}=\left(\begin{array}{ccc}
0 & -\left(\frac{\chi^{(\rho)}}{\alpha}\right)^{1 / 2} i k & 0 \\
-\left(\frac{\chi^{(\rho)}}{\alpha}\right)^{1 / 2} i k & -\left(\stackrel{\nu}{\ell}_{\ell}+\tilde{\omega}_{g}\right)^{2} k & \mathcal{M}_{1} \\
0 & \mathcal{M}_{2} & -\frac{2 \lambda}{3 n k_{B}} k^{2}
\end{array}\right)
$$

donde:

$$
\begin{aligned}
& \mathcal{M}_{1}=\frac{\left(\Gamma_{2}+\Gamma_{g}\right)}{n k_{B} T} \cdot\left(\frac{2}{3 \alpha}\right)^{1 / 2} i k^{3}-\chi^{(5)}\left(\frac{2}{3 \alpha}\right)^{1 / 2} i k, \\
& \mathcal{M}_{2}=\frac{\Gamma_{1}}{n k_{B} T}\left(\frac{2}{3 \alpha}\right)^{1 / 2} i k^{3}-\chi^{(5)}\left(\frac{2}{3 \alpha}\right)^{1 / 2} i k .
\end{aligned}
$$

La viscosidlad volumétrica generalizada, aparece en la diagonal de esta matriz y tiene una contribución a la viscosidad cortante longitudinal. En efecto,

$$
-\left(\check{\nu}_{\ell}+\tilde{\omega}_{g}\right) k^{2}=-\left(\frac{4}{3} \nu_{\ell}(\vec{k}, \omega)+\frac{\tilde{\omega}(k, \omega)}{n m}\right) k^{2},
$$


de acuerdo con las definiciones de las ecuaciones (5.19) y (5.20). Con el fin de encontrar la forma explícita de las matrices de la ecuación (5.1), vamos a considerar que la matriz de coeficientes de la ecuación (5.25) se puede descomponer formalmente como;

$$
\mathcal{L}_{i j}(\vec{k}, \omega)=i \Omega_{i j}(\vec{k})-\mathcal{K}_{i j}(\vec{k}, \omega)
$$

donde hemos utilizado una notación sugestiva para las componentes, las cuales representan la matriz de frecuencia y la matriz de memoria de nuestra descripción. Utilizando la ecuación (5.27) obtenemos,

$$
\Omega_{i j}(\vec{k})=\left(\begin{array}{ccc}
0 & -\left(\frac{\chi^{(\rho)}}{\alpha}\right)^{1 / 2} k & 0 \\
-\left(\frac{\chi^{(\rho)}}{\alpha}\right)^{1 / 2} k & 0 & -\left(\frac{2}{3 \alpha}\right)^{1 / 2} \chi^{(5)} k \\
0 & -\left(\frac{2}{3 \alpha}\right)^{1 / 2} \chi^{(5)} k & 0
\end{array}\right)
$$

para la matriz frecuencia, mientras que

$$
\mathcal{K}_{i j}(\vec{k}, \omega)=\left(\begin{array}{ccc}
0 & 0 & 0 \\
0 & \nu_{\ell} k^{2} & -\frac{\Gamma_{2}+\Gamma_{g}}{n k_{B} T}\left(\frac{2}{3 \alpha}\right)^{1 / 2} i k^{3} \\
0 & -\frac{\Gamma_{1}}{n k_{B} T}\left(\frac{2}{3 \alpha}\right)^{1 / 2} i k^{3} & \frac{2 \lambda}{3 n k_{B}} k^{2}
\end{array}\right)
$$

es la matriz de memoria, siendo todos los coeficientes de transporte funciones de $(\vec{k}, \omega)$. Además hemos definido,

$$
\begin{aligned}
\nu_{\ell}(\vec{k}, \omega) & =\stackrel{\circ}{\nu}_{\ell}(\vec{k}, \omega)+\tilde{\omega}_{g}(\vec{k}, \omega) \\
\Gamma_{2}(\vec{k}, \omega) & =\Gamma_{2}(\vec{k}, \omega)+\Gamma_{g}(\vec{k}, \omega) .
\end{aligned}
$$

Debido a que la componente transversal no se acopla con los flujos y ecuaciones de conservación longitudinales, consideramos por separado la componente transversal de las ecuaciones de balance. Utilizando el operador de proyección $\mathbb{Q}_{t}$. en las ecuaciones (5.6)-(5.8) obtenemos:

$$
\begin{aligned}
& -i \omega \rho \tilde{u}_{t_{\alpha}}-\rho \tilde{\omega}_{0}\left\{\frac{1}{2}(i k)^{2} \tilde{u}_{t_{\alpha}}\right\}+\chi^{(2)} \mathbb{Q}_{t} \cdot\left(i k_{j j} \stackrel{\stackrel{o}{P}}{K}_{i j}\right)- \\
& -\frac{\alpha \tilde{\omega}_{0}}{10} \cdot\left\{\frac{1}{2}(i k)^{2} \tilde{q}_{t_{\alpha}}^{K}\right\}=0 ; \alpha=1,2 .
\end{aligned}
$$


con ayuda de la relación,

$$
\begin{aligned}
\mathbb{Q}_{t} \cdot\left(i k_{j} \tilde{\stackrel{P}{P}}_{i j}\right)= & \chi^{(2)} \mathbb{Q}_{t} \cdot\left(i k_{j} \tilde{\stackrel{P}{P}}_{i j}^{K}\right)-\frac{1}{2} \rho \tilde{\omega}_{0}(i k)^{2} \tilde{u}_{t_{\alpha}} \\
& -\frac{1}{2} \frac{\tilde{\omega}_{0} \alpha}{10}(i k)^{2} \tilde{q}_{t_{\alpha}}^{K}
\end{aligned}
$$

que corresponde al flujo total de ímpetu transversal, podemos simplificar las ecuaciones de movimiento transversales, sustituyendo la ec. (5.34) en la ec. (5.33) de donde obtenemos,

$$
-i \omega \rho \tilde{u}_{t_{\alpha}}=-\mathbb{Q}_{t} \cdot\left(i k_{j} \tilde{P}_{i j}\right)_{\alpha}
$$

Considerando la relación constitutiva generalizada,

$$
\mathbb{Q}_{t} \cdot\left(i k_{j} \tilde{\stackrel{P}{P}}_{i j}\right)_{\alpha}=n m \nu_{t}(\vec{k}, \omega) k^{2} \tilde{u}_{t_{c \kappa}}
$$

correspondiente a la ecuación (4.49), y definiendo la velocidad de corriente transversal adimensional como,

$$
\tilde{u}_{t_{\alpha}}^{*}=\left(\frac{\alpha}{\chi^{(\rho)}}\right)^{1 / 2} \tilde{u}_{t_{\alpha}},
$$

podemos escribir la ecuación de movimiento en forma adimensional,

$$
-i \omega \tilde{u}_{t_{\alpha}}^{*}=-\nu_{t} k^{2} \tilde{u}_{t_{\alpha}}^{*} \quad \alpha=1,2
$$

La forma matricial de estas ecuaciones es;

$$
-i \omega \tilde{A}_{i}=\mathcal{T}_{i j} \tilde{A}_{j}
$$

la variable vectorial que aparece en esta ecuación se define como,

$$
\tilde{A}_{i}=\left(\begin{array}{c}
\tilde{u}_{t_{1}}^{*} \\
\tilde{u}_{t_{2}}^{*}
\end{array}\right) .
$$

La matriz de los coeficientes se puede separar formalmente de manera análoga a la ecuación (5.28),

$$
\mathcal{T}_{i j}(\vec{k}, \omega)=i \Omega_{i j}(\vec{k})-\mathcal{K}_{i j}(\vec{k}, \omega),
$$


y en este caso,

$$
\Omega_{i j}=\left(\begin{array}{cc}
0 & 0 \\
0 & 0
\end{array}\right), \quad \mathcal{K}_{i j}=\left(\begin{array}{cc}
\nu_{t} k^{2} & 0 \\
0 & \nu_{t} k^{2}
\end{array}\right) .
$$

En estas matrices la viscosidad cortante transversal generalizada tiene contribuciones de segundo orden en los flujos colisionales.

En una descripción unificada del comportamiento hidrodinámico, podemos formar una matriz por bloques para la matriz de memoria y la matriz frecuencia, utilizando las ecuaciones (5.29) para formar los elementos diagonales de una matriz de mayor tamaño. De hecho la parte transversal y la parte longitudinal se pueden poner en la misma matriz, de donde la matriz de memoria completa, tendrá la siguiente forma,

$$
\tilde{\mathcal{K}}=\left(\begin{array}{ccccc}
0 & 0 & 0 & 0 & 0 \\
0 & \nu_{\ell} k^{2} & \cdots & 0 & 0 \\
0 & \cdots & \cdots & 0 & 0 \\
0 & 0 & 0 & \nu_{t} k^{2} & 0 \\
0 & 0 & 0 & 0 & \nu_{t} k^{2}
\end{array}\right) .
$$

En la descripción que hemos hecho anteriormente, incorporamos nuestros resultados cinéticos de los coeficientes de transporte generalizados en el esquema general de la hidrodinámica generalizada, al construir explícitamente la matriz de frecuencia y el kernel de memoria.

Los principales resultados que hemos obtenido en este capítulo se encuentran en las ecs. (5.29) y (5.41), en relación con la forma explícita de la matriz frecuencia y la matriz de memoria del comportamiento hidrodinámico longitudinal y transversal, respectivamente. Los elementos de estas matrices hidrodinámicas son cantidades conocidas que provienen del modelo cinético Grad-Enskog. En particular, observamos que la matriz frecuencia depende explícitamente de la densidad y de la velocidad térmica molecular, mientras que los elementos de la matriz de memoria son esencialmente los coeficientes de transporte generalizados, que en esta descripción representan la respuesta no-local y no-instantánea del sistema ante variaciones de las variables relevantes.

Debemos notar que nuestra deducción constituye solamente el punto de partida, para estudiar la región finita de vectores de onda y frecuencias, hacia la hidrodinámica generalizada dentro del esquema general de funciones 
de correlación. Esta es una observación muy importante en relación con la interpretación de las ecuaciones de movimiento, más allá del equilibrio local, debido a que representan la influencia de las variables rápidas sobre las variables conservadas.

Por otro lado, es notable el hecho de que la matriz de memoria, ec. (5.43), es simétrica; esto es consecuencia directa de la simetría que existe en los efectos cruzados de transporte en las relaciones constitutivas generalizadas. Notemos que para obtener una matriz de memoria simétrica, debemos considerar la contribución a los procesos de relajación de la viscosidad volumétrica generalizada y del coeficiente térmico de disipación $\Gamma_{g}$, los cuales aparecen en la relación constitutiva dada por la ecuación (5.20). Esta simetría se obtiene también en la descripción cinética que solamente toma en cuenta fujos colisionales a primer orden y naturalmente en el límite del gas diluido, sin embargo, no es tan obvio predecir que esta cualidad de simetría se cumpla al incluir flujos colisionales de orden superior. La simetría de la matriz de memoria es importante, porque sugiere razonablemente desarrollar la teoría de fluctuaciones para demostrar, al menos parcialmente, alguna simetría general tipo Onsager, no obstante, todas las aproximaciones y limitaciones de la teoría de Enskog. 


\section{Conclusiones y Perspectivas.}

Hemos desarrollado un modelo cinético para predecir el comportamiento hidrodinámico generalizado de un gas moderadamente denso de esferas duras, a partir de la teoría estandar de Enskog.

Las ecuaciones cinéticas tipo Enskog son las más utilizadas para describir la evolución temporal de la función de distribución de una partícula en gases densos monatómicos. La ecuación de Enskog para gases formados por moléculas que interactúan a través de un potencial de esfera dura constituye el punto de partida para el modelo cinético. Las aproximaciones de Enskog conducen a flujos colisionales de ímpetu y energía, así como a una ecuación de transferencia para los momentos de la función de distribución que incluye flujos cinéticos y flujos colisionales. Estos últimos se pueden desarrollar en serie de Taylor dando origen a diferentes aproximaciones en potencias del diámetro molecular. El origen, evaluación e incorporación de los flujos debidos a la transferencia colisional dentro del esquema cinético de Grad-Enskog, constituye el principal elemento que se presenta al tratar con gases densos monatómicos.

Para resolver la ecuación de Enskog, utilizamos el método de Grad en la aproximación de 13-momentos el cual a pesar de su similitud con gases diluidos tiene diferencias y complicaciones muy importantes cuando se requiere evaluar los flujos colisionales de orden superior. En la aproximación anterior el tensor de presiones y el flujo de calor son variables relevantes del sistema de la misma categoría que las variables conservadas. En efecto, el método de momentos de Grad nos proporciona el conjunto de ecuaciones de movimiento para los flujos, las cuales tienen la forma de ecuaciones de relajación con tiempos característicos que dependen de la densidad. Las ecuaciones de movimiento constituyen el punto inicial para el cálculo de los coeficientes de transporte generalizados, y como ya hemos mencionado contienen términos que provienen de la contribución por transferencia colisional a los flujos.

En este trabajo hemos considerado por separado la aproximación a primer orden y a segundo orden en los flujos colisionales. En ambos casos al expresar el tensor de presiones y el flujo de calor en términos de los gradientes de las variables conservadas, llegamos a un conjunto de relaciones constitutivas generalizadas en el espacio de Fourier. Un estudio detallado del mecanismo de transferencia colisional nos permite incorporar flujos por transferencia colisional de orden superior en las relaciones constitutivas generalizadas. 
Formalmente los coeficientes generalizados que se obtienen para gases densos de Enskog, a primer orden en los flujos colisionales, son muy parecidos a los del gas diluido de esferas duras. Esto ocurre principalmente en su dependencia con respecto al vector de onda y a la frecuencia. Sin embargo, el modelo cinético incorpora en estas expresiones algunos factores de renormalización no triviales que dependen de la densidad a través de la función de correlación por pares en equilibrio local. Los coeficientes de transportes generalizados son funciones analíticas del vector de onda y la frecuencia que tienen una expresión en fracciones continuas.

Sabemos que cualquier modelo hidrodinámico generalizado debe tener en el límite apropiado el comportamiento que predice la hidrodinámica usual. En efecto, los coeficientes de transporte generalizados se reducen a los coeficientes de transporte usuales que se obtienen mediante el método de ChapmanEnskog, en el límite de longitudes de onda larga y bajas frecuencias. Así mismo, en el límite de bajas densidades recuperamos los resultados conocidos para los coeficientes generalizados en gases diluidos. De hecho, tomando esto como referencia, discutimos las modificaciones de las curvas para los coeficientes de transporte al variar independientemente el vector de onda y la frecuencia, para diferentes densidades reducidas en donde es válida la ecuación de Enskog. Los resultados que obtenemos están en concordancia con los correspondientes a los cálculos de Dinámica Molecular, sin embargo, una mejor tendencia de las curvas a vectores de onda grande se obtiene solamente cuando incluimos flujos colisionales de orden superior. Los resultados muestran un comportamiento típico para las funciones de memoria como función del vector de onda y la frecuencia el cual está determinado, principalmente, por los tiempos de relajación asociados con los flujos.

Posteriormente, hemos extendido el modelo cinético Grad-Enskog para incluir en la descripción flujos colisionales de segundo orden en los gradientes. En principio, salvo pequeñas correcciones a los coeficientes generalizados que eventualmente pudieran ser importantes, ningún cambio considerable se obtiene en los resultados anteriores. No obstante, la incorporación de flujos colisionales a segundo orden en los gradientes en la diagonal del tensor de presiones, permite hallar una viscosidad volumétrica generalizada que tiene un comportamiento interesante con respecto a variaciones en el vector de onda. En efecto, aún cuando desciende normalmente al eje horizontal cerca del régimen hidrodinámico, independientemente de la densidad tiende rápidamente a un valor casi constante dejando un residuo de memoria para 
valores grandes del vector de onda. Esta característica se observa también para otros coeficientes generalizados en la misma aproximación, y está de acuerdo con el comportamiento de los resultados de dinámica molecular. En el límite de bajas densidades la viscosidad volumétrica tiende a cero mientras que en el régimen hidrodinámico recuperamos los resultados conocidos que se obtienen mediante el método de Chapman-Enskog.

Para poner en contacto el tratamiento cinético con el esquema usual de funciones de correlación de la hidrodinámica generalizada, construimos la matriz frecuencia y la matriz de memoria correspondientes al esquema fenomenológico de la ecuación de Langevin generalizada en el espacio de Fourier. Los coeficientes de transporte aparecen como elementos de la matriz de memoria y en la aproximación a segundo orden en los gradientes sobresale el hecho de que la matriz de memoria es simétrica. Este resultado ya se había presentado anteriormente en la aproximación a primer orden en los flujos colisionales y naturalmente en gases diluidos.

El desarrollo anterior permite plantear algunas perspectivas más o menos inmediatas que pueden servir como revisión y aplicación de este trabajo. Con ayuda de la forma explícita de los coeficientes de transporte generalizados, podemos construir las ecuaciones hidrodinámicas válidas en muchas aplicaciones donde la matriz de memoria solamente se propone con modelos empíricos razonables. En este sentido podemos proseguir a evaluar las soluciones de las ecuaciones hidrodinámicas generalizadas bajo restricciones específicas que impongan las aplicaciones concretas. En particular podemos pensar en los modos hidrodinámicos colectivos que nos proporcionen un indicio sobre los rasgos principales de los espectros de absorción-dispersión de luz y neutrones para sistemas densos. Concretamente podemos calcular el factor de estructura dinámico por medio de los eigenvalores de la matriz hidrodinámica que hemos encontrado, para ver cuales son las modificaciones que resultan en un sistema denso, al mismo tiempo que podríamos hacer una comparación semi-cuantitativa con los experimentos de dispersión de neutrones y dinámica molecular.

Debido a que el método de momentos de Grad se aplica a sistemas densos con modelos moleculares más complicados, este tratamiento abre la posibilidad de utilizarse en otras ecuaciones cinéticas tipo-Enskog. Por ejemplo, ecuaciones asociadas con gases densos de esfera rugosas y mezclas binarias. De tal forma que podemos desarrollar expresiones para los coeficientes de transporte generalizados adecuados para tales sistemas. 
Los resultados que hemos obtenido para los coeficientes de transporte generalizados tienen un comportamiento adecuado cerca del régimen hidrodinámico, por lo tanto, las ecuaciones de evolución de las variables relevantes a segundo orden en los flujos por transferencia colisional nos permiten estudiar diferentes aproximaciones en el número de Knudsen. Así, podemos estudiar eventualmente relaciones de Burnett para los gases de Enskog de manera mucho más sistemática. 


\section{Apéndices}

\section{Apéndice A}

\section{Flujos Colisionales}

Supongamos que $\psi$ es la propiedad molecular para la que nos interesa calcular el flujo. Esta función se expresa como una cantidad que depende de la velocidad molecular. A hora consideramos la transferencia de la cantidad $\psi$ a través de un elemento de área $d S$ de un plano tal como $P P^{\prime}$ el cual pasa por algún punto intermedio $\vec{x}$ entre los centros moleculares de las partículas que chocan.

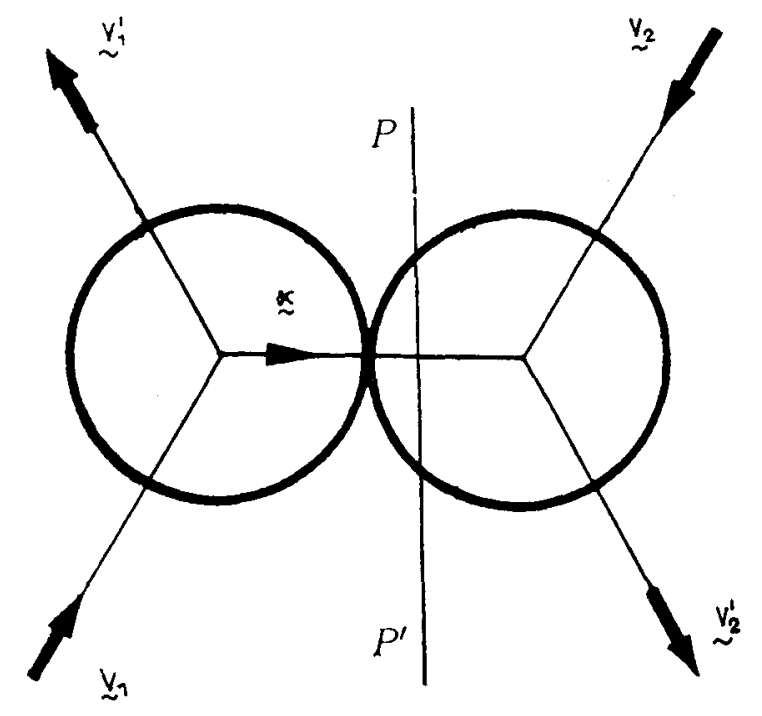

Figure 9. Geometría de una colisión binaria 
Para comenzar, supodremos que $\psi$ es una cantidad invariante en colisiones, para el caso de gases monoatómicos en el modelo de esferas duras los invariantes son:

$$
\psi=m, m \vec{c}, \frac{1}{2} m c^{2} .
$$

En el caso de colisiones binarias, la conservación de los invariantes $\psi^{(i)}$, $i=1,2, \ldots, 5$ se expresa como:

$$
\left(\psi^{(i)^{\prime}}+\psi_{1}^{(i)^{\prime}}-\psi^{(i)}-\psi_{1}^{(i)}\right)=0 .
$$

Las colisiones no alteran la cantidlad total de $\psi$ de las dos moléculas, sino solamente transfiere parte de esta cantidad de una molécula a la otra, de donde va a resultar un flujo por transferencia colisional.

Sí en una colisión se produce una transferencia de $\psi$ a través del área $d S$, contenida en el plano $P P^{\prime}$, los centros cle las moléculas en colisión están en lados opuestos de esta superficie y la línea que los une la corta en forma transversal.

Supongamos que la normal a $d S$ está definida por $\hat{n}$, y como $\hat{k}$ apunta hacia la molécula de referencia entonces $(\hat{k} \cdot \hat{n}) \geq 0$. Si la línea de longitud " $a$ " que une los centros 01 en colisión corta a $d S$, el centro de la molécula 1 debe estar dentro de un cilindro con base $d S_{k}$ y generadores paralelos a $\hat{k}$ de longitud " $a$ ". Aquí $d S_{k}$ es la componente de la superficie orientada $d S$ a lo largo de $\hat{k}$, es decir, $d S_{k} \equiv d S(\hat{k} \cdot \hat{n})$. El volumen de este cilindro es: $\operatorname{ad} S_{k}=\operatorname{adS}(\hat{k} \cdot \hat{n})$.

Por otro lado, si el punto de contacto está en $\vec{x}$ entonces la posición de los centros de las moléculas 0,1 en colisión, están en los puntos $\vec{x} \pm \frac{1}{2} a \hat{k}$ respectivamente. De donde, "La probabilidad por unidad de tiempo de una colisión tal que el centro de la primera molécula esté dentro del volumen $d \vec{x}=\operatorname{adS}(\hat{k} \cdot \hat{n})$, las velocidades de las moléculas antes del choque estén en los intervalos $d \vec{c}, d \vec{c}_{1}$ alrededor de $\vec{c}, \vec{c}_{1}$ respectivamente, y que la dirección $\hat{k}$ de la línea de los centros 0,1 se encuentre en el intervalo d $\hat{k}$ alrededor de $\hat{k} "$ es:

$$
\chi(\vec{x}) f\left(\vec{x}+\frac{a}{2} \hat{k}\right) f_{1}\left(\vec{x}-\frac{a}{2} \hat{k}\right) a^{2}(\vec{g} \cdot \hat{k}) d \hat{k} d \vec{c}_{1} d \vec{c} a(\hat{k} \cdot \hat{n}) d S
$$

En cada colisión de este tipo, una molécula del lado positivo de $d S$ gana una cantidad $\left(\psi^{\prime}-\psi\right)$ de la propiedad $\psi$ a expensas de la otra molécula del lado negativo. Multiplicando la ecuación anterior por $\left(\psi^{\prime}-\psi\right)$, e integrando 
adecuadamente, obtenemos la razón total de transferencia colisional de $\psi$ a través de $d S$ por unidad de tiempo y unidad de área; $\vec{\phi}_{\text {col }} \cdot \hat{n}$, donde $\vec{\phi}_{\text {col }}$, es un vector que representa la contribución de colisiones al flujo de $\psi$, debido a las colisiones moleculares, este flujo por transferencia colisional (o por potencial) está dado por:

$$
\vec{\phi}_{\mathrm{col}_{i}} \equiv \frac{a^{3}}{2} \chi(\vec{x}) \int\left(\psi^{\prime}-\psi\right) f\left(\vec{x}-\frac{a}{2} \hat{k}\right) f_{1}\left(\vec{x}+\frac{a}{2} \hat{k}\right) k_{i}(\vec{g} \cdot \hat{k}) d \hat{k} d \vec{c} d \vec{c}_{1}
$$

con la restricción de que:

$$
(\vec{g} \cdot \hat{k}) \geq 0 .
$$

Desarrollando las funciones de distribución en serie de Taylor alrededor del punto $\vec{x}$, y reteniendo los dos primeros términos de este clesarrollo, obtenemos para $\phi_{\text {col }_{i}}$ la aproximación siguiente:

$$
\begin{gathered}
\phi_{\mathrm{col}_{i}} \cong \phi_{i}^{I}+\phi_{i}^{I I} \\
\phi_{i}^{I}=\frac{a^{3}}{2} \chi \int\left(\psi^{\prime}-\psi\right) f f_{1} k_{i}(\vec{g} \cdot \hat{k}) d \hat{k} d \vec{c} d \vec{c}_{1} \\
\phi_{i}^{I I}=\frac{a^{4}}{4} \chi \int\left(\psi^{\prime}-\psi\right) f f_{1} \frac{\partial}{\partial x_{j}}\left(\ln \frac{f}{f_{1}}\right) k_{i} k_{j}(\vec{g} \cdot \hat{k}) d \hat{k} d \vec{c} d \vec{c}_{1} .
\end{gathered}
$$

Las funciones $\chi, f$ y $f_{1}$ están evaluadas en $\vec{x}$ y sumamos sobre los índices repetidos. Notemos que para evaluar las contribuciones de los fujos colisionales solamente se requiere conocer la función de distribución de una partícula, es decir, necesitamos resolver la ecuación de Enskog. También cabe mencionar que el flujo macroscópico total de la cantidad $\psi$ está dado por:

$$
\vec{\phi}_{\text {cinetico }}+\vec{\phi}_{\text {colisional }}=\vec{\phi}_{\text {total }} \text {. }
$$




\section{Apéndice B}

\section{Integrales constitutivas}

Los momentos cinéticos de orden superior se calculan como en gases diluidos y por lo tanto no entraremos en su discusión $[38,39]$. Los flujos por transferencia colisional son de mayor interés para el problema que estamos considerando. A primer orden por ejemplo, tenemos:

$$
\begin{aligned}
P_{i j}^{I} & =\frac{a^{3}}{2} \chi m \int f f_{1}\left(C_{i}^{\prime}-C_{i}\right) k_{j}(\vec{g} \cdot \hat{k}) d \hat{k} d \vec{c}_{1} d \vec{c} \\
& =\frac{a^{3}}{2} \chi m \int f f_{1} k_{i} k_{j}(\vec{g} \cdot k)^{2} d \hat{k} d \vec{c}_{1} d \vec{c}
\end{aligned}
$$

donde hemos utilizado,

$$
C_{1}^{\prime}-\bar{C}_{i}=(\vec{g} \cdot \hat{k}) k_{i} .
$$

La integral con respecto a $d \hat{k}$ puede expresarse en términos de la velocidad relativa molecular $g_{i}=C_{1 i}-C_{i}$, como:

$$
\int k_{i} k_{j}(\vec{g} \cdot \hat{k})^{2}=\frac{2 \pi}{15}\left(2 g_{i} g_{j}+g^{2} \delta_{i j}\right),
$$

de donde

$$
\begin{aligned}
P_{i j}^{I} & =\frac{a^{3}}{2} \chi m \frac{2 \pi}{15} \int f f_{1}\left(2\left(C_{1 i}^{\prime}+C_{1 j}+C_{i} C_{j}\right)+\left(C_{1}^{2}+C^{2}\right) \delta_{i j}\right) d \vec{c}_{1} d \vec{c} \\
& =\frac{a^{3}}{2} \frac{\chi}{m} \frac{2 \pi}{15}\left(4 \rho P_{i j}+2 \rho \frac{3 k_{B} T}{m} \delta_{i j}\right),
\end{aligned}
$$

$\operatorname{con} b=\frac{2 \pi a^{3}}{3 m}$.

Simplificando esta expresión obtenemos,

$$
P_{i j}^{I}=\left(\frac{2}{5} \stackrel{\rho}{P}_{i j}^{K}+\frac{k_{B} \rho T}{m} \delta_{i j}\right) \chi b \rho .
$$

Esta expresión corresponde a la representación del tensor de presiones a 13-momentos en la aproximación a primer orden en los flujos colisionales. 
Otro ejemplo es,

$$
P_{i j k}^{I}=\frac{a^{3}}{2} \chi m \int f f_{1}\left(C_{i}^{\prime} C_{j}^{\prime}-C_{i} C_{j}\right) k_{k}(\vec{g} \cdot \hat{k}) d \hat{k} d \vec{c}_{1} d \vec{c}
$$

sustituyendo la relación,

$$
C_{i}^{\prime} C_{j}^{\prime}-C_{i} C_{j}=C_{i} k_{j}(\vec{g} \cdot \hat{k})+C_{j} k_{i}(\vec{g} \cdot \hat{k})+k_{i} k_{j}(\vec{g} \cdot \hat{k})^{2},
$$

obtenemos,

$$
\begin{aligned}
P_{i j k}^{I} & =\frac{a^{3}}{2} \chi m \int f f_{1} C_{i} k_{j} k_{k}(\vec{g} \cdot \hat{k})^{2} d \hat{k} d \vec{c}_{1} d \vec{c} \\
& +\frac{a^{3}}{2} \chi m \int f f_{1} C_{j} k_{i} k_{k}(\vec{g} \cdot \hat{k})^{2} d \hat{k} d \vec{c}_{1} d \vec{c} \\
& +\frac{a^{3}}{2} \chi m \int f f_{1} k_{i} k_{j} k_{k}(\vec{g} \cdot \hat{k})^{3} d \hat{k} d \vec{c}_{1} d \vec{c}
\end{aligned}
$$

puesto que la última integral tiene simetría impar, se anula y no contribuye.

Definiendo

$$
\mathcal{Y}_{i j k}=\frac{a^{3}}{2} \chi m \int f f_{1} C_{i} k_{j} k_{k}(\vec{g} \cdot \hat{k})^{2} d \hat{k} d \vec{c}_{1} d \vec{c}
$$

podemos escribir la ecuación (B.8) como,

$$
P_{i j k}^{I}=\mathcal{Y}_{i j k}+\mathcal{Y}_{j i k}
$$

Usando la ec. (B.3), es posible evaluar la integral $\mathcal{Y}_{i j k}$; en efecto,

$$
\begin{aligned}
\mathcal{Y}_{i j k} & =\frac{a^{3}}{2} \chi m \frac{2 \pi}{15} \int f f_{1} C_{i}\left(2 g_{i} g_{j}+g^{2} \delta_{j k}\right) d \vec{c}_{1} d \vec{c} \\
& =\frac{a^{3}}{2} \chi m \frac{2 \pi}{15} \int f f_{1} C_{i}\left(2 C_{j} C_{k}+C^{2} \delta_{j k}\right) d \vec{c}_{1} d \vec{c} \\
& =\left(\frac{2}{5}\left(q_{i} \delta_{j k}+q_{j} \delta_{i k}+q_{k} \delta_{i j}\right)+q_{j} \delta_{i k}\right) \frac{\chi b \rho}{5} .
\end{aligned}
$$

Intercambiando índices

$$
\mathcal{Y}_{j i k}=\frac{2}{25}\left(\left(q_{i} \delta_{j k}+q_{j} \delta_{i k}+q_{k} \delta_{i j}\right)+q_{j} \delta_{i k}\right) \chi b \rho .
$$


Sustituyendo las ecuaciones (B.11)-(B.12) en la ecuación (B.10) obtenemos,

$$
P_{i j k}^{I}=\frac{9}{25}\left(q_{i} \delta_{j k}+q_{j} \delta_{i k}+\frac{4}{9} q_{k} \delta_{i j}\right) \chi b \rho
$$

y así sucesivamente.

Para evaluar integrales constitutivas de segundo orden en los flujos por transferencia colisional, necesitamos sustituir la función de distribución a 13-momentos, un ejemplo específico es el siguiente,

$$
P_{i j}^{I I}=\frac{1}{4} \chi a^{4} \int f f_{1} m\left(C_{i}^{\prime}-C_{i}\right) k_{j} k_{k} \frac{\partial}{\partial x_{k}}\left(\ln \frac{f}{f_{1}}\right)(\vec{g} \cdot \hat{k}) d \vec{c}_{1} d \vec{c}
$$

Utilizando la forma general de $f^{(13)}$,

$$
f^{(13)}=f^{(0)}(1+X)
$$

en la ecuación anterior, obtenemos en la aproximación lineal;

$$
\begin{aligned}
P_{i j}^{I I} & =\frac{1}{4} \chi a^{4} \int f f_{1} m\left(C_{i}^{\prime}-C_{i}\right) k_{j} k_{k} \frac{\partial}{\partial x_{k}}\left(\ln \frac{f^{(0)}}{f_{1}^{(0)}}\right)(\vec{g} \cdot \hat{k}) d \vec{c}_{1} d \vec{c} \\
& +\frac{1}{4} \chi a^{4} \int f f_{1} m\left(C_{i}^{\prime}-C_{i}\right) k_{j} k_{k} \frac{\partial}{\partial x_{k}}\left(X-X_{1}\right)(\vec{g} \cdot \hat{k}) d \vec{c}_{1} d \vec{c} \\
& =\mathcal{Y}_{i j}^{(1)}+\mathcal{Y}_{i j}^{(2)}
\end{aligned}
$$

donde,

$$
\begin{aligned}
X-X_{1}= & \frac{m}{2 k_{B}^{2}} \cdot \frac{\stackrel{\circ}{P}_{i j}^{K}}{n T^{2}}\left(C_{i} C_{j}-C_{1 i} C_{i j}\right)+\frac{m}{k_{B}^{2}} \frac{q_{i}^{K}}{n T^{2}}\left(C_{1 i}-C_{i}\right) \\
& +\frac{m^{2}}{5 k_{B}^{3}} \cdot \frac{q_{i}^{K}}{n T^{3}}\left(C^{2} C_{i}-C_{1}^{2} C_{1 i}\right) .
\end{aligned}
$$


Evaluamos cada integral por separado

$$
\begin{aligned}
\mathcal{Y}_{i j}^{(1)}= & \frac{1}{4} \chi a^{4} \int f^{(0)} f_{1}^{(0)} m\left(C_{i}^{\prime}-C_{i}\right) k_{j} k_{k} \frac{\partial}{\partial x_{k}}\left(\frac{m}{2 k_{B} T}\left(C_{1}^{2}-C^{2}\right)\right) \\
& \cdot(\vec{g} \cdot \hat{k}) d \hat{k} d \vec{c}_{1} d \vec{c} \\
= & \frac{1}{4} \chi a^{4} \int f^{(0)} f_{1}^{(0)} m\left\{\frac{m}{2 k_{B} T^{2}}\left(C^{2}-C_{1}^{2}\right)\right\} \int \frac{\partial T}{\partial x_{k}} k_{i} k_{j} k_{k}(\vec{g} \cdot \hat{k}) d \hat{k} d \vec{c}_{1} d \vec{c} \\
& +\frac{1}{4} \chi a^{4} \int f^{(0)} f_{1}^{(0)} m\left\{\frac{m}{2 k_{B} T^{2}}\left(C_{\ell}-C_{\ell_{1}}\right)\right\} \int \frac{\partial u_{\ell}}{\partial x_{k}} k_{i} k_{j} k_{k}(\vec{g} \cdot \hat{k})^{2} d \hat{k} d \vec{c}_{1} d \vec{c},
\end{aligned}
$$

y utilizando la relación siguiente [44],

$$
\int k_{i} k_{j} k_{k}(\vec{g} \cdot \hat{k})^{2} d \hat{k}=\frac{2 \pi}{24} g^{-1}\left[g\left(g_{i} \delta_{j k}+g_{j} \delta_{i k}+g_{k} \delta_{i j}\right)+g_{i} g_{j} g_{k}\right],
$$

obtenemos,

$$
\begin{aligned}
\mathcal{Y}_{i j}^{(1)}= & \frac{1}{4} \chi a^{4}\left\{\frac{\pi}{24} \frac{m^{2}}{k_{B} T^{2}}\right\} \int f^{(0)} f_{1}^{(0)}\left(C^{2}-C_{1}^{2}\right) g\left(g_{i} \frac{\partial T}{\partial x_{j}}+g_{j} \frac{\partial T}{\partial x_{i}}\right) d \vec{c}_{1} d \vec{c} \\
& +\frac{1}{4} \chi a^{4}\left\{\frac{\pi m^{2}}{24 k_{B} T^{2}}\right\} \int f^{(0)} f_{1}^{(0)}\left(C^{2}-C_{1}^{2}\right) g^{-1}\left(g_{i} g_{j}+g^{2} \delta_{i j}\right) g_{k} \frac{\partial T}{\partial x_{k}} d \vec{c}_{1} d \vec{c} \\
& +\frac{1}{4} \chi a^{4}\left\{\frac{\pi m^{2}}{12 k_{B} T^{2}}\right\} \int f^{(0)} f_{1}^{(0)}\left(C_{\ell}-C_{1 \ell}\right) g\left(g_{i} \frac{\partial u_{\ell}}{\partial x_{j}}+g_{j} \frac{\partial u_{\ell}}{\partial x_{i}}\right) d \vec{c}_{1} d \vec{c} \\
& +\frac{1}{4} \chi a^{4}\left\{\frac{\pi m^{2}}{12 k_{B} T}\right\} \int f^{(0)} f_{1}^{(0)}\left(C_{\ell}-C_{1 \ell}\right) g^{-1}\left(g_{i} g_{j}+g^{2} \delta_{i j}\right) g_{k} \frac{\partial u_{\ell}}{\partial x_{k}} d \vec{c}_{1} d \vec{c} .
\end{aligned}
$$

Recordando que,

$$
f^{(0)} f_{1}^{(0)}=n^{2}\left(\frac{m}{2 \pi k_{B} T}\right)^{3} e^{-\frac{m G_{0}^{2}}{k_{B} T}-\frac{m g^{2}}{4 k_{B} t}}
$$

donde,

$$
G_{0 i}=\frac{1}{2}\left(C_{1 i}+C_{i}\right)
$$


y tomando en cuenta las propiedades del Jacobiano cuando se realiza un cambio de coordenadas,

$$
d \vec{c}_{1} d \vec{c}=d \vec{G}_{0} d \vec{g} .
$$

obtenemos por sustitución directia de las ecuaciones (B.19)-(B.21),

$$
\mathcal{Y}_{i j}^{(1)}=-\tilde{\omega}\left\{\frac{6}{5}\left(\frac{\partial u_{i}}{\partial x_{j}}\right)^{0}+\frac{\partial u_{k}}{\partial x_{k}} \delta_{i j}\right\}
$$

siendo,

$$
\tilde{\omega}=\frac{4}{9} \chi a^{4} n^{2} \pi^{1 / 2}\left(m k_{B} T\right)^{1 / 2}
$$

la viscosidad volumétrica usual del régimen hidrodinámico.

Por otro lado,

$$
\begin{aligned}
\mathcal{Y}_{i j}^{(2)}= & \frac{1}{4} \chi a^{4} \int f^{(0)} f_{1}^{(0)} m\left(C_{i}^{\prime}-C_{i}\right) k_{j} k_{k}\left\{\frac{m}{2 n k_{B}^{2} T^{2}} \cdot \frac{\partial \stackrel{\circ}{i}_{i j}^{K}}{\partial x_{k}}\left(C_{i} C_{j}-C_{1 i} C_{1 j}\right)\right\} \\
& \cdot(\vec{g} \cdot \hat{k})^{2} d \hat{k} d \vec{c}_{1} d \vec{c}+ \\
+ & \frac{1}{4} \chi a^{4} \int f^{(0)} f_{1}^{(0)} \cdot m\left(C_{i}^{\prime}-C_{i}\right) k_{j} k_{k}\left\{\frac{m}{n k_{B}^{2} T^{2}} .\right. \\
& \left.\cdot \frac{\partial q_{i}^{K}}{\partial x_{k}}\left(\frac{m}{5 k_{B} T}\left(C^{2} C_{i}-C_{1}^{2} C_{i}\right)+\left(C_{1 i}-C_{i}\right)\right)\right\}(\vec{g} \cdot \hat{k})^{2} d \hat{k} d \vec{c}_{1} d \vec{c},
\end{aligned}
$$

utilizando la ecuación (B.2) y reordenando el integrando,obtenemos;

$$
\begin{aligned}
\mathcal{Y}_{i j}^{(2)}= & \frac{1}{4} \chi a^{4} \int f^{(0)} f_{1}^{(0)} m\left\{\frac{m}{2 n k_{B}^{2} T^{2}}\left(C_{\ell} C_{s}-C_{1 \ell} C_{1 s}\right)\right\} \int \frac{\partial \stackrel{P}{\ell}_{\ell}^{K}}{\partial x_{k}} k_{i} k_{j} k_{k}(\vec{g} \cdot \hat{k})^{2} d \hat{k} d \vec{c}_{1} d \vec{c} \\
+ & \frac{1}{4} \chi a^{4} \int f^{(0)} f_{1}^{(0)} m\left\{\frac{m}{n k_{B}^{2} T^{2}}\left(\frac{m}{5 k_{B} T}\left(C^{2} C_{\ell}-C_{1}^{2} C_{1 \ell}\right)+\left(C_{1 \ell}-C_{\ell}\right)\right)\right\} \\
& \times \int \frac{\partial q_{\ell}^{K}}{\partial x_{k}} k_{i} k_{j} k_{k}(\vec{g} \cdot \hat{k})^{2} d \hat{k} d \vec{c}_{1} d \vec{c} .
\end{aligned}
$$


Realizando la integral respecto a $d \hat{k}$, con ayuda de la ecuación (B.17):

$$
\begin{aligned}
\mathcal{Y}_{i j}^{(2)}= & \frac{1}{4} \chi a^{4}\left\{\frac{\pi}{12 n}\left(\frac{m}{k_{B} T}\right)^{2}\right\} \int f^{(0)} f_{1}^{(0)}(-g)\left(\frac { m } { 5 k _ { B } T } \left(2 g_{r} G_{0 r} G_{0 \ell}+G_{0}^{2} g_{\ell}+\right.\right. \\
& \left.\left.+\frac{1}{4} g^{2} g_{\ell}\right)-g_{\ell}\right)\left(g_{i} \frac{\partial q_{\ell}^{K}}{\partial x_{j}}+g_{j} \frac{\partial q_{\ell}^{K}}{\partial x_{i}}\right) d \vec{c}_{1} d \vec{c}+ \\
& +\frac{1}{4} \chi a^{4}\left\{\frac{\pi}{12 n}\left(\frac{m}{k_{B} T}\right)^{2}\right\} \int f^{(0)} f_{1}^{(0)}\left(-g^{-1}\right)\left(\frac { m } { 5 k _ { B } T } \left(2 g_{r} G_{0 r} G_{0 \ell}+G_{0}^{2} g_{\ell}+\right.\right. \\
& \left.\left.+\frac{1}{4} g^{2} g_{\ell}\right)-g_{\ell}\right)\left(g_{i} g_{j}+g^{2} \cdot \delta_{i j}\right) g_{k} \frac{\partial q_{\ell}^{K}}{\partial x_{k}} d \vec{c}_{1} d \vec{c} .
\end{aligned}
$$

Sustituyendo las ecuaciones (B.19)-(B.21) y simplificando con ayuda de las relaciones de la ec. (B.23) obtenemos;

$$
\mathcal{Y}_{i j}^{(2)}=\frac{-\tilde{\omega}}{10 n m}\left(\frac{m}{k_{B} T}\right)\left\{\frac{6}{5}\left(\frac{\partial q_{i}^{K}}{\partial x_{j}}\right)^{0}+\frac{\partial q_{k}^{K}}{\partial x_{k}} \delta_{i j}\right\},
$$

donde $\tilde{\omega}$ ha sido definida anteriormente. Por último, los cálculos se pueden resumir como sigue;

$$
\begin{aligned}
P_{i j}^{I I}= & \mathcal{Y}_{i j}^{(1)}+\mathcal{Y}_{i j}^{(2)} \\
= & -\tilde{\omega}\left\{\frac{6}{5}\left(\frac{\partial u_{i}}{\partial x_{j}}\right)^{0}+\frac{\partial u_{k}}{\partial x_{k}} \delta_{i j}\right\}- \\
& -\frac{\tilde{\omega}}{10 m m}\left(\frac{m}{k_{B} T}\right)\left\{\frac{6}{5}\left(\frac{\partial q_{i}^{K}}{\partial x_{j}}\right)^{0}+\frac{\partial q_{k}^{K}}{\partial x_{k}} \delta_{i j}\right\}
\end{aligned}
$$

Así sucesivamente, de manera similar se pueden evaluar las otras integrales constitutivas. 


\section{Apéndice $\mathrm{C}$}

\section{Simetría de la matriz de memoria}

A segundo orden en los flujos colisionales, para que la matriz de memoria $\mathcal{K}_{i j}(\vec{k}, \omega)$ sea simétrica se requiere que:

$$
\Gamma_{1}=\Gamma_{2}+\Gamma_{d} T
$$

Esta condición se obtiene inmediatamente al comparar los elementos fuera de la diagonal de la matriz de memoria, ec.(5.31), y utilizando la definición del coeficiente cruzado que aparece en la ec.(5.20). Los términos a cada lado de la igualdad en la ec.(C.1), tienen la forma explícita siguiente;

$$
\begin{aligned}
\Gamma_{1}(\vec{k}, \omega)= & \frac{8}{9} \frac{\chi^{(3)} c_{v} T \tau_{p} \tau_{q}\left(\chi^{(2)} \chi^{(3)} n k_{B} T-\frac{1}{25}-\frac{9}{4} \cdot \tilde{\omega} \tilde{\omega}_{0} k^{2}\right)}{\left(1-i \omega \tau_{q}+\tilde{\omega}_{0} \tau_{q} k^{2}\right)} \epsilon_{\alpha, a d}^{K}(\vec{k}, \omega) \\
& +\frac{8}{15} \chi^{(2)} c_{v} T \tilde{\omega} \tau_{p}\left[1+\frac{3 \cdot 9}{8 \cdot 4} \cdot \frac{\chi^{(3)}}{\chi^{(2)}} \frac{\left(1-i \omega \tau_{p}+\frac{1}{2} \tilde{\omega}_{0} \tau_{p} k^{2}\right)}{\left(1-i \omega \tau_{q}+\tilde{\omega}_{0} \tau_{q} k^{2}\right)}\right] \epsilon_{\alpha, a d}^{K}(\vec{k}, \omega) \\
\Gamma_{2}(\vec{k}, \omega)= & \frac{8}{9} \frac{\chi^{(3)} c_{v} T \tau_{p} \tau_{q}\left(\chi^{(2)} \chi^{(3)} n k_{B} T-\frac{1}{25} \tilde{\omega} \tilde{\omega}_{0} k^{2}\right)}{\left(1-i \omega \tau_{q}+\tilde{\omega}_{0} \tau_{q} k^{2}\right)} \epsilon_{\alpha, a d}^{K}(\vec{k}, \omega) \\
& +\frac{8}{15} \chi^{(2)} c_{v} T \tilde{\omega} \tau_{p}\left[1+\frac{3}{8} \frac{\chi^{(3)}}{\chi^{(2)}} \frac{\left(1-i \omega \tau_{p}+\frac{1}{2} \tilde{\omega}_{0} \tau_{p} k^{2}\right)}{\left(1-i \omega \tau_{q}+\tilde{\omega} \tau_{q} k^{2}\right)}\right] \epsilon_{\alpha, a d}^{K}(\vec{k}, \omega)
\end{aligned}
$$

Notemos

$$
\Lambda_{\alpha, a d}^{K}(\vec{k}, \omega)=\frac{\left(1-i \omega \tau_{p}+\frac{1}{2} \tilde{\omega}_{0} \tau_{p} k^{2}\right)}{\left(1-i \omega \tau_{q}+\tilde{\omega}_{0} \tau_{q} k^{2}\right)} \epsilon_{\alpha, a d}^{K}(\vec{k}, \omega)
$$

también,

$$
\begin{aligned}
\Gamma_{d} T= & \frac{1}{6} \chi^{(3)} c_{v} T \tilde{\omega} \tau_{q}\left[1-\frac{4}{15} \frac{\tilde{\omega} \tau_{p} k^{2}}{\left(1-i \omega \tau_{p}+\frac{1}{2} \tilde{\omega}_{0} \tau_{p} k^{2}\right)}\right] \times \\
& \times \frac{\left(1-i \omega \tau_{p}+\frac{1}{2} \tilde{\omega}_{0} \tau_{p} k^{2}\right)}{\left(1-i \omega \tau_{q}+\tilde{\omega}_{0} \tau_{q} k^{2}\right)} \epsilon_{\alpha, a d}^{K}(\vec{k}, \omega)
\end{aligned}
$$




$$
\begin{aligned}
\Gamma_{d} T & =\frac{8}{9} \chi^{(3)} c_{v} T \tau_{p} \tau_{q}\left[\frac{-\tilde{\omega} \tilde{\omega}_{0} k^{2}}{20\left(1-i \omega \tau_{q}+\tilde{\omega}_{0} \tau_{q} k^{2}\right)}\right] \cdot \epsilon_{\alpha, a d}^{K}(\vec{k}, \omega) \\
& +\frac{8}{15} \chi^{(2)} c_{v} \tilde{\omega} \tau_{p}\left[\frac{15}{8} \cdot \frac{1}{4} \cdot \frac{\chi^{(3)}}{\chi^{(2)}} \frac{\left(1-i \omega \tau_{p}+\frac{1}{2} \tilde{\omega}_{0} \tau_{p} k^{2}\right)}{\left(1-i \omega \tau_{q}+\tilde{\omega}_{0} \tau_{q} k^{2}\right)}\right] \epsilon_{\alpha, a d}^{K}(\vec{k}, \omega)
\end{aligned}
$$

Escrito de esta manera se puede hacer la suma de los coeficientes $\Gamma$-as, teniendo en cuenta los términos comunes de estas expresiones. De donde,

$$
\begin{aligned}
\Gamma_{2}+\Gamma_{d} T= & \frac{8}{9} \frac{\chi^{(3)} c_{v} T \tau_{p} \tau_{q}\left(\chi^{(2)} \chi^{(3)} n k_{B} T-\left(\frac{1}{25} \cdot \frac{9}{4}\right) \tilde{\omega} \tilde{\omega}_{0} k^{2}\right)}{\left(1-i \omega \tau_{q}+\tilde{\omega}_{0} \tau_{q} k^{2}\right)} \eta_{\alpha, a d}^{K}(\vec{k}, \omega) \\
& +\frac{8}{15} \chi^{(2)} c_{v} T \tilde{\omega} \tau_{p}\left[1+\frac{3}{8}\left(\frac{9}{4}\right) \frac{\chi^{(3)}}{\chi^{(2)}} \frac{\left(1-i \omega \tau_{p}+\frac{1}{2} \tilde{\omega} \tau_{p} k^{2}\right)}{\left(1-i \omega \tau_{q}+\tilde{\omega}_{0} \tau_{q} k^{2}\right)}\right] \eta_{\alpha, a d}^{K}(\vec{k}, \omega) \\
= & \Gamma_{1} .
\end{aligned}
$$

Los elementos fuera de la diagonal de la matriz de memoria son iguales. Por lo tanto la matriz es simétrica. 


\section{Bibliografía}

[1] Boon, J.P., Yip, S., Molecular Hydrodynamics, Dover Pub. Inc. NY (1991).

[2] Hansen, J.P.Mc Donald, I.R., Theory of simple liquids, 2nd. ed. Academic Press Inc., London (1986).

[3] Evans, J.D., Morris, G.P., Statistical Mechanics of Non-equilibrium liquids, Academic Press Inc., London, (1990).

[4] March, N.H., Tosi, M.P., Atomic Dynamics in liquids, Dover Pub. Inc. N.Y., (1976).

[5] Brenig, W., Statistical Theory of Heat, Springer-Verlag, Berlin, (1989).

[6] Jou, D., Casas-Vázquez, J., Lebon, C., Extended Irreversible Thermodynamics, Springer-Verlag, Berlin, (1993).

[7] Wang, C.H., Spectroscopy of Condensed Media Dynamic of Molecular Interactions, Academic Press. Inc. London, (1985).

[8] Résibois, P., De Leener, H., Classical Kinetic Theory of fluids, John Willey \& Sons, N.Y., (1977).

[9] Lovesey, S.W., Springer, T., Dynamic of Solids and liquids by Neutron Scattering, Springer-Verlag, N.Y. (1977).

[10] van Well, A.A., Verkerk, P., de Graaf, L.A., Suck, J.-B., Copley, J.R.D., Density fluctuations in liquid argon: Coherent dynamic structure factor along the $120 K$ isotherm obtained by neutron scattering, Phys. Rev. A. 1 (1985), 3391.

[11] van Well, A.A., P., de Graaf, L.A., Density fluctuations in liquid neon studied by neutron scattering, Phys. Rev. A. 32 (1985), 2396.

[12] Wester huijs, P., de Graaf, L.A., de Schepper, I.M., Half widths of neutron spectra in dense helium-neon gas mixtures, Phys. Rev. E. 48, (1993), 1948. 
[13] Copley, J.R.D., Rowe, J.M., Density fluctuations in liquid rubidium, I. Neutron-Scattering meassurments, Phys. Rev. A 9 (1974), 1656.

[14] de Schepper, M., Verkerk, P., van Well, A.A., de Graaf, L.A., Short wave lenght sound modes in Liquid Argon, Phys. Rev. Lett. 50 (1983), 974.

[15] Hoover, Wm. G., Non equilibrium molecular dynamics: the first 25 years, Physica A 194 (1993), 450.

[16] Koplik, J., Banavar, J.R., Continuum deductions from molecular Hydrodynamic, Ann. Rev. Fluid Mech. 27 (1995) 257.

[17] Cohen, E.G.D., Kamgar-Parsi, B., Generalized Hydrodinamic and eigen modes for a dense hard sphere fluid, Phys. Lett. 144 A, (1986) 241 .

[18] de Schepper, I.M., van Rijs, U.C., van Well, A.A., Varkerk, P., de Graaf, L.A., Microscopic Sound waves in dense Lennard-Jones fluids, Phys. Rev. A. 39 (1989) 259.

[19] Kambayashi, S., Hi watari, Y., Molecular-Dynamic study of dynamical properties of dense soft-sphere fluids: The role of short- range repulsion of the intermolecular potential, Phys. Rev. E. 49 (1994) 1251.

[20] Mryglod, I.M., Omelyam, I.P., Tokarchuk, M.V., Generalized Collective modes for the Lennard-Jones fluid, Molecular Physics 84 (1995) 235 .

[21] L'Hevereux, I., Oppenheim, I., Generalized Hydrodynamics of a nonequilibrium steady State fluid in a Large temperature gradient, Physica A 148 (1988) 503.

[22] Rosenau, P., Extending hydrodynamics via the regularization of ChapmanEnskog expansion, Phys. Rev. A 40 (1989) 7193.

[23] Lutsko, J.F., Dufty, J.W., Das, S.P., Fluctuations and dissipation in a fluid under shear: Linear Dynamics, Phys. Rev. A 39 (1989) 1311.

[24] Zhu, S.B., Lee, J., Robinson, G.W., Microscopic friction in ultra fast dynamical processes, Phys. Rev. A 39 (1989) 5985. 
[25] Das, S.P., Dufty, J.W., Hydrodynamic dispersion relations in dense fluids, Phys. Rev. A 46 (1992) 6371.

[26] Bertolini, D., Tani, A., Generalized Hydrodynamic and the acoustic modes of water: Theory an simulation results, Phys. Rev. E. 51 (1995) 1091.

[27] de Graaf, L.A., de Scheper, I.M., Short-wave lenght collective excitations in Liquids, J. Phys. Condens matter 2 (1990) SA99.

[28] Bafile, V., Nerkerk, P., de Graaf, L.A., Barocchi, F., Suck, J-B., Mytka, H., Onset of Departure from linearized Hydrodynamic Behavior in Argon Gas studied with Neutron Brillouin Scattering, Phys. Rev. Lett. 65 (1990) 2394.

[29] Achivat, T. Boukenter, A., Duval, E., Fick, B., García, N. Serughetti, J., Comparision between light and neutron inelastic Scatterings. The frecuency linear behavior of the light-vibration coupling coefficient, Physica A 201 (1993) 257.

[30] Kamgar-Parsi, B., Cohen, E.C.D., de Schepper, I.M., Dynamical processes in hard-sphere fluids, Phys. Rev. A 35 (1987) 4781.

[31] Enciso, E., Almarza, N.G., del Prado, V., Bermejo, F..J. Zapata, E.L., Ujaldón, M., Molecular-dynamic simulation on simple fluids: Departure from linearized hydrodynamic behavior of the dynamical structure factor, Phys. Rev. E 50 (1994) 1336.

[32] Alley, W.E., Alder, B.J., Generalized transport coefficients for hard spheres, Phys. Rev. A 27 (1983) 3158.

[33] Ullo, J.J., Yip, S., Molecular clynamics of dense gases: Effects of continuous potentials, Phys. Rev. A 29 (1984) 2092.

[34] Balucani, U., Vallauri, R., Gaskell, T., Transverse current and generalized shear viscosity in liquid rubidium, Phys. Rev. A 35 (1987) 4263.

[35] Levesque, D., Weis, J.J., Vermesse, J. Computation of transport coefficients and the dynamical structure factor of $X e$ in the presence of Long-range three-body interactions, Phys. Rev. A 37 (1988) 918. 
[36] Mareschal, M., Mansour, M.M., Sonnino, G., Kestemant, E., Dynamic structure factor in a non equilibrium fluid: A molecular dynamics approach, Phys. Rev. A 45 (1992) 7180.

[37] Kirkpatric, T.R., Short-wavelenght collective modes and generalized hydrodynamic equations for hard spheres particles, Phys. Rev. A 32 (1985) 3130.

[38] Velasco, R.M., García Colín, L.S., Generalized Hydrodynamics in Gases, J. Non-Equilib. Thermodyn. 20 (1995) 1.

[39] Velasco, R.M., García Colín, L.S., Kinetic Approach to Generalized Hydrodynamics, Phys. Rev. A 44 (1991) 4961.

[40] Cohen, E.G.D., Fifty years of kinetic theory, Physica A 194 (1993), 229.

[41] Cohen, E.G.D., Kinetic theory: Understanding nature through collisions, Am. J. Phys.61 6 (1993) 524.

[42] Leegwater, J.A., Kinetic theory of Dense fluids, Doctoral Disertion (1991).

[43] Leegwater, J.A., Kinetic theory of Lennard-Jones fluids, J. Chem. Phys. 95 (1991) 8346.

[44] Chapman, S., Cowling, T.G., The Mathematical Theory of Non-Uniform Gases, $3^{\text {rd. }}$ ed., Cambridge University Press, Cambridge 1970.

[45] Ferziger, J.H., Kaper, H.G., Mathematical Theory of Transport Processes in Gases, North Holand, Amsterdam, 1972.

[46] Hirschfelder, J.O., Curtiss, C.F., Bird, R.B., Molecular Theory of Gases and Liquids, J. Wiley \& Sons, $2^{\text {nd. }}$ ed., 1974.

[47] Velasco, R.M., Teoría Cinética de gases, memorias EMFE (1993).

[48] Dorfman, J.R., van Beijeren, H., The Kinetic Theory of Gases, statistical Mechanics, Part B: Time Dependent Processes, ed. B.J. Berne, Plenum, 1977. 
[49] van Beijeren, H., Kinetic Theory of dense gases and Liquids, Fundamental Problems in Statistical Mechanics VII, Elsevier Sc. Pub. (1990).

[50] Velarde, M.G., On the Enskog hard-sphere Kinetic equation and the transport phenomena of dense simple gases, Lecture Notes in Physics 31, Springer, Berlin (1974) 288.

[51] Amorós, J., Maeso, M.J., Villar, E., A Test of the Modified Enskog Theory for the transport properties of liquids, Int. J. Thermophys. 13 (1992) 907.

[52] Robert, M., Jeng, J.F., Viswanathan, R., On the density expansion of the pair distribution function of non uniform fluids. J. Chem. Phys. 88 (1988) 1983.

[53] Reed, T.M., Gubbins, K.E., Thermodynamic and transport properties of fluids, App. Stat. Mech., Chemical-Enginering, Series McGraw Hill, (1973).

[54] Kremer, G.M., Rosa, Jr. E., On Enskog's dense gas theory. I. The method of moments for monatomic gases, J. Chem. Phys. 89 (1988) 3240 .

[55] Reinecke, S., Kremer, G.M., Method of moments of Grad, Phys. Rev. A. 42 (1990) 815.

[56] Gaio, D.C., Kremer, G.M., Kinetic Theory for Polyatomic Dense Gases of Rough Spherical Molecules, J. Non-Equilib. Thermodyn. 16 (1991) 357.

[57] Arkeryd, L., Cercignani, C., Global Existence in $L^{1}$ for the Enskog Equation and convergence of the solution to solutions of the Boltzmann Equation, J. Stat. Phys. 62 (1990) 845.

[58] Cannone, M., Cercignani, C., The inverse conjecture for the Revised Enskog Equation, J. Stat. Phys. 63 (1991) 363.

[59] Grad, H., Principles of the Kinetic Theory of Gases, Thermodynamics in Gases, Handbuch der Physik, Vol. 12, Springer-Verlag, Berlin (1958). 
[60] Grad, H., On the kinetic Theory of Rarefied Gases, Commun. Pure. Appl. Math. 2 (1994), 331.

[61] Schmidt, G., Köhler, W.E., Hess, S., On the kinetic theory of the Enskog Fluid. Viscosity and Viscoelasticity, Heat conduction and thermal Pressure, Z. Naturforsch 369 (1981) 545. (Desarrollo de gases densos de $f$ ).

[62] Rangel-Huerta, A. , Velasco, R.M., Generalized Transport coefficients in Enskog Gases, J. Non-equilib. Thermodyn. 21 (1996) 41.

[63] Velasco, R.M., García Colín, L.S., The Kinetic foundations of extended irreversible thermodynamics, Revisted, J. Stat. Phys. 69 (1992) 217.

[64] García Colín, L.S. Flores, A., On the transport coefficients of Mode rately dense gases, physica 32 (1966) 289.

[65] Hoffman, D.K., Curtiss, C.F., Kinetic theory of Dense Gases. IV. Transport Virial Coefficients, Phys. Fluids 8 (1965) 667.

[66] Gray, P., Rice, S.A., On the kinetic theory of Dense fluids. XVIII. The Bulk Viscosity, J. Chem. Phys. 41 (1964) 3689.

[67] Zwanzig, R., Frequency-Dependent transport Coefficient in Fluid Mechanics, J. Chem. Phys. 43 (1965) 714.

[68] Zwanzig, R., Mountain, R.D., High-Frequency elastic Moduli of Simple Fluids, J. Chem. Phys. 43 (1965) 4464.

[69] Komarov, L.I. Contribution th the theory of the bulk viscosity coefficient, Soviet Phys. JETP 21 (1965), 99.

[70] Romanov, V.P., Ul'yanov, S.V., Bulk viscosity in relaxing media, Physica A 201 (1993) 527.

[71] Xu, J., Stell, G., Bulk and Shear viscosity of a polydisperse hard sphere fluid, J. Stat. Phys. 57, (1989), 921.

[72] Hoheisel, C., Bulk viscosity of model fluids. A comparison of equilibrium and non-equilibrium molecular dynamics results, J. Chem. Phys. 86, (1987), 2328. 
[73] Hoheisel, C., Vogelsang, R., Schoen, M., Bulk viscosity of LennardJones fluid for a wide range of states computed by equilibrium molecular dynamics, J. Chem. Phys. 87, (1987), 7195.

[74] Hanley, H.J.M., Cohen, E.G.D., Analysis of the transport coefficients for simple dense fluids: The diffusion and bulk viscosity coefficients, Physica 83 A, (1976), 215.

[75] García-Colín, L. S., Brownian motion and its implication in non-equilibrium statistical mechanics, Kinam 1, (1979), 107. 